\title{
Abstracts of the 41st European Muscle Conference
}

\section{1-5 Sept 2012, Rhodes, Greece}

(C) The Author(s) 2012. This article is published with open access at Springerlink.com

The following abstracts were submitted for consideration in response to the call for abstracts to the three general thematics of the 41st EMC,

- Structure function (track 1)

- Systems biology \& epigenetics (track 2)

- Clinical \& technological transfer (track 3)

covering all aspects of muscle research with an emphasis given to translational research. Also, the conference programme is enriched by the participation of the European Research Networks "MUZIC" and "MYOAGE" which organise two respective symposia. Final programming was underway at the time this went to print by the $41 \mathrm{st}$ EMC's International Scientific Board (ISB) and the organizers (visit www.emc2012.gr). The abstracts were reviewed by an independent Abstracts Committee, members or the ISB and the organizers, who also edited the abstracts.

\section{1st EMC Organizers}

Christina Karatzaferi, University of Thessaly, GR Georgios K. Sakkas, CERETETH, GR

\section{1st EMC International Scientific Board}

Michael A. Geeves, University of Kent, UK, chair Masataka Kawai, University of Iowa, USA co-chair Anders Arner, Karolinska Institute, SWE (ex officio) Gillian Butler-Browne, Institute of Myology, FRA Roger Cooke, University of California San Francisco, USA Kristina Djinovic-Carugo, University of Vienna, AT Mathias Gautel, King's College London, UK Anne Houdusse, Institute Curie, FRA

Costis N. Maganaris, Manchester Metropolitan University, UK Alf Mansson, Linnaeus University, SWE

Kathryn H. Myburgh, University of Stellenbosch, SA

Marco Narici, University of Nottingham, UK
Gabriele Pfitzer, University of Cologne, DE Corrado Poggesi, University of Florence, IT Håkan Westerblad, Karolinska Institute, SWE Susan Wray, University of Liverpool, UK

\section{1st EMC Abstracts Committee}

Steven Marston, Imperial College London, UK chair Marieke Bloemnik, University of Kent, UK, co-chair Nikos Pinotsis, University of Vienna, AU, co-chair Pradeep K. Luther, Imperial College London, UK Christoforos Giannaki, University of Nicosia, CY Konstantina Dipla, Aristotle University of Thessaloniki, GR Ioannis Vogiatzis, National and Kapodistrian University of Athens, GR

Gregory C. Bogdanis, National and Kapodistrian University of Athens, GR

Yiannis Koutedakis, University of Thessaly, GR

Gianis Giakas, University of Thessaly, GR

Athanasios Jamurtas, University of Thessaly, GR

Vassilis Paschalis, University of Thessaly, GR

We thank all contributors for their hard work! CK \& GKS

Acknowledgments The organisers thank all contributors for their hard work. The 41st EMC was placed under the auspices of the University of Thessaly and of the Center for Research Technology Thessaly (CERETETH), Greece. Moreover, the organisers would like to express their thanks to the following Academic Partners: the EU Framework 7 Projects MYOAGE and MUZIC, the Journal of Muscle Research and Cell Motility, and Supporters: DTM, WPI- SI Heidelberg, Aurora Scientific Inc., IonOptix, MyoTronic, Biomechanical Solutions. 


\section{Key Note Lecture}

\section{Control of Muscle Growth and Muscle Metabolic Function}

\section{S. Schiaffino}

Venetian Institute of Molecular Medicine (VIMM), Padova, Italy

In the first part of this lecture I will discuss current work in my lab on a novel regulatory pathway involved in muscle gene regulation and muscle growth in adult skeletal muscle. In the second part I will discuss our work on intrinsic and extrinsic control of circadian rhythms in skeletal muscle, with particular reference to metabolic control.

The 41st EMC Keynote Lecture is sponsored by MYOAGE.

\section{Track 1: Structure \& function}

\section{1}

Effects of 2-deoxy-ATP binding on the pre- and post- powerstroke states of myosin

S.G Nowakowski ${ }^{1}$, N. Adamek ${ }^{2}$, M.A. Geeves ${ }^{2}$, V.Daggett ${ }^{1}$, M. Regnier ${ }^{1}$

${ }^{1}$ University of Washington, Department of Bioengineering, Seattle, WA, USA; ${ }^{2}$ University of Kent, School of Biosciences, Canterbury, UK

We previously demonstrated that substituting 2-deoxy-ATP (dATP) for ATP increases the rate and magnitude of force production in demembranated cardiac muscle and intact cardiomyocytes. We used molecular dynamics (MD) simulations to study the pre- and postpowerstroke states to investigate the dATP-induced structural changes responsible for this functional enhancement.

Dictyostelium discoideum S1 crystal structures for pre- (PDB: $1 \mathrm{VOM}$ ) and post- (PDB ID: 1MMA) powerstroke myosin were simulated with (d)ADP, $\left(\mathrm{P}_{\mathrm{i}}\right)$, and $\mathrm{Mg}^{2+}$ for $50 \mathrm{~ns}$. Solution biochemistry kinetics was used to validate model predictions of the nucleotide influence on crossbridge binding and detachment. dADP binding to myosin resulted in allosteric alterations to the actin binding surface that increased polar residue exposure. This change in surface charge may increase electrostatic interaction between myosin and actin and enhance binding prior to the myosin powerstroke. The binding pocket itself was also altered as a result of dADP binding; myosin spent less time in contact with dADP than with ADP as a result of a conformational shift that increased the distance between the nucleotide and the binding pocket. These data indicate that dADP may be released more rapidly than ADP in the post-powerstroke state, thus increasing the rate of crossbridge detachment.

While kinetic experiments with fast and $\operatorname{slow}(\beta)$ rabbit skeletal acto-S1 showed $30 \%$ weaker affinity for dADP than for ADP, the rate of ADP and AADP release from acto-S1 $(\beta)$ was similar. Interestingly, studies of S1 showed that dADP was released $30 \%$ faster than ADP. Further simulation studies and biochemical analyses are ongoing.

\section{3}

Expression of myogenesis-related genes during early-postnatal muscle development in the pig

D. Lösel, C. Rehfeldt, C. Kalbe

Leibniz Institute for Farm Animal Biology (FBN), Research Unit Muscle Biology and Growth, Dummerstorf, Germany
Myogenesis was considered to be restricted to the prenatal period in the pig. However, we found a substantial increase in total fibre number in the semitendinosus muscle during the early postnatal period (Bérard et al. 2011). We hypothesized that the formation of a tertiary generation of myofibres is involved in this phenomenon.

As a first step we investigated whether the increase in fibre number is reflected by changes in the expression of myogenesis-associated genes using qPCR. Semitendinosus muscle of German Landrace piglets was examined at days $1,7,21$, and 28 of age ( $n=5$ per age). The decreased expression of MyHC-emb at d 21 and d $28(P<0.001)$ compared with d 1 may be associated with a decreased frequency of tertiary myofibres. PAX7 mRNA expression was lower at d 21 and 28 $(P<0.1)$ compared with $\mathrm{d} 1$ indicating a reduced number of satellite cells. MYOD specific mRNA expression was not affected by age, whereas MYF5 was already decreased at d $7(P=0.07)$. MYOG mRNA expression was decreased $(P=0.07)$ only at $\mathrm{d} 21$. MRF4 mRNA was reduced at $\mathrm{d} 7(P<0.05)$ compared with $\mathrm{d} 1$. In addition, myogenic precursor cells, differentiating myogenic cells, and tertiary fibres were localized in muscle cross sections using immunohistochemistry to investigate the origin of newly formed fibres.

The results suggest a decreasing rate of determination and subsequent differentiation of cells during the period of the early postnatal increase in fibre number.

\section{References}

Bérard J, Kalbe C, Lösel D, Tuchscherer A, Rehfeldt C (2011) Histochem Cell Biol 136:217-225.

\section{4}

Frank-Starling's law of the heart: myofilament length dependent activation

P.P de Tombe

Loyola University Chicago, Stritch School of Medicine, Department of Cell and Molecular Physiology, Maywood, IL 60153, USA

The Frank-Starling law of the heart describes the interrelationship between end-diastolic volume and cardiac ejection volume, a regulatory system that operates on a beat-to-beat basis. At the cellular level, sarcomere length dependent myofilament $\mathrm{Ca}^{2+}$ sensitivity underlies this phenomenon that is termed myofilament length dependent activation (LDA). How the contractile apparatus transduces the information concerning SL is not known. That is, although the Frank-Starling Law of the Heart constitutes a fundamental property of the heart that has been appreciated for well over a century, the molecular mechanisms that underlie this phenomenon are still incompletely understood.

Our research is aimed to enhance our understanding of this important physiological process that controls cardiac performance on a beat-to-beat basis and to elucidate the molecular mechanisms that underlie LDA. A unifying theory has been proposed that LDA may be due to changes in the spacing between the thick and thin filament; however, through a comprehensive series of experiments, we have found that changes in inter-filament spacing may not be the primary mechanism that underlies LDA. On the other hand, cardiac troponin-I, and in particular the threonine-144 residue, is both essential and sufficient to impart LDA properties onto the cardiac sarcomere. Furthermore, we found that interruption of cooperative activation along the thin filament markedly enhances LDA, while a reduction in active cycling cross-bridges does not affect LDA. Recent x-ray diffraction based structural studies show a direct linkage between diastolic sarcomere length and the ordering of myosin, troponin, and myosin binding protein $\mathrm{C}$.

Together, our findings suggest that the molecular mechanisms that underlie length dependency in muscle are the result of a direct 
sarcomere length mediated modulation of the structure/function of the thin filament system, myosin and/or thick filament system, or the kinetics/structure of the interaction between actin and myosin.

\section{8}

Fast and slow cardiac myosin isoforms differ in rates of MgADP release and MgATP binding

D.W. Maughan ${ }^{1}$, Y. Wang ${ }^{1}$, B.C.W. Tanner ${ }^{1}$, A.T. Lombardo ${ }^{1}$, S.M. Tremble ${ }^{2}$, P. VanBuren ${ }^{1,2}$, M.M. LeWinter ${ }^{1,2}$, B.M. Palmer ${ }^{1}$

${ }^{1}$ University of Vermont, Department of Molecular Physiology and Biophysics, VT05405 USA; ${ }^{2}$ University of Vermont, Department of Medicine, Burlington, VT 05405, USA

Species of origin and various physiological and pathological conditions drive the relative content of the two cardiac myosin heavy chain (MyHC) isoforms expressed in the mammalian heart.

We measured myosin cross-bridge detachment rate and the corresponding rates of MgADP release and MgATP binding in myocardial strips from mice and rats fed an iodine-deficient, propylthiouracil diet resulting in $\sim 100 \%$ expression of $\beta$-MyHC in the ventricles. Ventricles of control animals expressed $\sim 100 \%$ $\alpha$-MyHC. Chemically-skinned myocardial strips prepared from papillary muscle were subjected to sinusoidal length perturbation analysis at maximum calcium activation and $17{ }^{\circ} \mathrm{C}$. Frequency characteristics of myocardial viscoelasticity were used to calculate cross-bridge detachment rate at various MgATP concentrations (0.01-5 mM).

The rate of MgADP release, equivalent to the asymptotic value of cross-bridge detachment rate at high $[\mathrm{MgATP}]$, was progressively slowed according to species and isoform in the order: mouse $\alpha$-MyHC $>$ rat $\alpha$-MyHC $>$ mouse $\beta$-MyHC $>\beta$-MyHC (range $111.4 \pm$ $6.2-15.5 \pm 0.8 \mathrm{~s}^{-1}$ ), whereas the order for the rate of $\operatorname{MgATP}$ binding was mouse $\alpha$-MyHC $>$ mouse $\beta$-MyHC $>$ rat $\alpha$-MyHC $>$ rat $\beta$-MyHC (range $325 \pm 32-55 \pm 6 \mathrm{mM}^{-1} \mathrm{~s}^{-1}$ ).

Because the amino acid sequence of the nucleotide binding pocket is identical for these four myosins, we infer that MgADP release and MgATP binding is determined by unique isoform- and speciesspecific amino acid differences in other regions of the myosin molecule. We postulate that corresponding differences in the stiffness profile of myosin cause the nucleotide binding pocket to be deformed differentially, thereby producing differences in strain-sensitivity of nucleotide release and binding.

\section{9}

Training interventions reverse dislocation of nNOS in chronically painful muscle

L. Jensen ${ }^{1}$, U. Frandsen ${ }^{2}$, A.L. Mackey ${ }^{3}$, L.L. Andersen ${ }^{4}$, G. Sjøgaard ${ }^{2}$

${ }^{1}$ University of Southern Denmark, Institute of Clinical Research, Clinical Pathology, Odense, Denmark; ${ }^{2}$ University of Southern Denmark, Institute of Sports Science and Biomechanics, Muscle Physiology and Biomechanics, Odense, Denmark; ${ }^{3}$ University of Copenhagen, Department of Orthopedic Surgery M, Institute of Sports Medicine, Copenhagen, Denmark; ${ }^{4}$ University of Southern Denmark, Institute of Sports Science and Biomechanics, Physical Activity and Health at Work, Odense, Denmark

Chronic pain in the trapezius muscle is frequent in females with repetitive work tasks. Physical exercise has been shown to reduce pain and improve muscle function, but the underlying mechanisms are unclear. Nitric oxide (NO) signaling within the skeletal muscle has proven very important in modulating cellular function. A decrease in or dislocation of neuronal nitric oxide synthase (nNOS) could lead to dysfunctional regulation of $\mathrm{NO}$ causing ineffective muscle activation and poor oxygenation.

This randomized study investigated the effect on nNOS of two different training interventions to improve trapezius muscle function. 35 women clinically diagnosed with trapezius myalgia (MYA) and 18 healthy controls $(\mathrm{CON})$ were included. The subjects with trapezius myalgia were assigned to either 10 weeks of (1) general fitness training (GFT, $n=15$ ) or (2) specific strength training (SST, $n=18$ ).

Women with MYA showed sarcoplasmic nNOS localization in $20 \%$ of muscle fibers compared to $13 \%$ in CON $(P<0.05)$. Furthermore, fibers with loss of sarcolemmal nNOS were all type I and significantly larger than the mean CSA $(P<0.001)$. In addition, SST resulted in a decrease of sarcoplasma-localized nNOS after training (21\%-11\%; $P<0.05)$, while GFT also decreased $(17-11 \%)$ although not significantly.

With this study we show that trapezius myalgia displays loss of sarcolemma-localized nNOS and increased sarcoplasma-localized nNOS, and that sarcoplasma-localized nNOS in skeletal muscle can be decreased with exercise. Our findings suggest that the trapezius myalgia muscle has impaired local NO signaling due to loss of sarcolemma-localized nNOS, which may underlie previous findings of impaired muscle oxygenation during repetitive work tasks.

\section{2}

Toad heart utilizes exclusively slow skeletal muscle troponin $\mathbf{T}$ : a unique evolutionary adaptation and functional benefits

\section{H.Z. Feng, M.M. Hossain X. Chen, J.P. Jin}

Wayne State University, School of Medicine, Department of Physiology, Detroit, MI 48201, USA

In all species studied to date, the three isoforms of vertebrate troponin $\mathrm{T}(\mathrm{TnT})$ are expressed in a muscle type-specific manner. We have discovered an exception that the cardiac muscle of toad (Bufo) expresses exclusively slow skeletal muscle $\mathrm{TnT}$ ( $\mathrm{ss} \operatorname{TnT}$ ) together with cardiac forms of troponin I and myosin as determined using immunoblotting and/or LC-MS/MS.

Using RT-PCR and $3^{\prime}$ - and $5^{\prime}$-RACEs on toad cardiac mRNA, we cloned full length cDNAs encoding two alternatively spliced variants of ssTnT. Expression of the cloned cDNAs in E. coli confirmed that the toad cardiac muscle expresses solely ssTnT, predominantly the low molecular weight variant with the exon 5-encoded $\mathrm{NH}_{2}$-terminal segment spliced out. Functional studies were performed in ex vivo working toad hearts and compared with the frog (Rana) hearts. The results showed that toad hearts had higher contractile and relaxation velocities and were able to work against a significantly higher afterload than that of frog hearts. Therefore, the unique evolutionary adaptation of utilizing exclusively ssTnT in toad cardiac muscle corresponded to a fitness value from improving systolic function of the heart.

The data demonstrated a physiological importance of the functional diversity of TnT isoforms. The structure-function relationship of TnT may be explored for the development of new treatment of heart failure.

\section{4}

New structural units in the actin cross linking protein filamin $\mathrm{C}$

\section{R. Sethi, J. Ylänne}

University of Jyväskylä, Department of Biological and Environmental Science and Nanoscience Center, Jyväskylä, Finland 
The dynamic nature of actin cytoskeleton is essential for cell division, adhesion, migration, signal transduction and various other cellular processes. The nucleation, polymerization and organization of the actin filament network is mediated by a number of proteins. Filamins are actin cross-linking proteins composed of two $290 \mathrm{kDa}$ subunits. Each subunit is composed of $\mathrm{N}$ terminal actin binding domain followed by a flexible rod region containing 24 Immunoglobulin-like domains.

We have earlier found that in the C-terminal rod-2 part of filamin there are three closely interacting pairs of immunoglobulinlike domains. Two of these domains pairs can serve as mechanically regulated binding sites for integrins, other transmembrane receptors and for cytoplasmic adaptor proteins. This provides a way how mechanical contraction of actomyosin cytoskeleton can regulate cell adhesion and signalling. To find out whether other areas of filamins have similar structures we expressed all possible two immunoglobulin-like domain fragments of the rod-1 region from human Filamin C.

We found three compact two-domain pairs. Of these, we have crystallized the fragment consisting of immunoglobulin domains 4 and 5. This fragment shows a novel type of domain-domain interaction, where the two domains form a loop-like structure with large interaction surface.

While the previously characterized domain pairs of the filamin rod 2 region interact through the edge of a beta-sheet, the domains 4-5 interact face-to-back along their beta-sheets. This kind of structure could be easily opened by mechanical force and we are currently investigating if this provides a new functional site in filamins.

Marie Curie Initial Training Network - Muscle Z disk Protein Complexes (MUZIC).

Sethi is a candidate for a young investigator award to be judged during the EMC 2012.

\section{6}

\section{A novel myopathic condition caused by mutation of the MACF1} gene locus

L.H. Jørgensen ${ }^{1}$, M.M. Jensen ${ }^{2}$, N. Færgemann ${ }^{2}$, J. Graakjær ${ }^{3}$, S.V. Jacobsen ${ }^{4}$, H.D. Schrøder ${ }^{1}$

${ }^{1}$ University of Southern Denmark, Institute of Clinical Health, SDU muscle research cluster, Odense, DK; ${ }^{2}$ University of Southern Denmark, Institute of Biochemistry and Molecular Biology, Odense, DK; ${ }^{3}$ Vejle Hospital, Clinical Genetics Department, Vejle, DK;

${ }^{4}$ Esbjerg Hospital, Paediatrics, Esbjerg, DK

The cytoskeleton is a key factor in basic functions common to all cell types. It defines the shape and size of a given cell, is very dynamic and responds to environmental signaling cues. Information transfer occurs via cytoskeletal proteins such as spectrins and plakins, molecular giants linking cytoskeletal components to each other and to junctions. Microtubule-actin cross-linking factor 1 (MACF1) is a member of the spectraplakin family and a ubiquitously expressed $614 \mathrm{kDa}$ protein. MACF1 is distributed in a cytoplasmic filamentous network where it is alpha and omega in controlling microtubule dynamics. The phenotype of MACF1-/-mice is developmental retardation and embryonic death at gastrulation stage e7.5, displaying a "head without trunk" phenotype with similarities to WNT3-/-mice. These observations suggest a fundamental role for MACF1 in development and pinpoint the essentiality of the protein.

Using Array-Comparative Genomic Hybridization, immunohistochemistry, RT-PCR, transmission electron microscopy and western blotting we have analyzed a patient with severe muscle weakness.

We discovered that the muscle weakness was associated with reduced expression of MACF1 mRNA and protein and caused by a one-allelic duplication of the MACF1 locus. Using C. Elegans as a model we validated that reduced expression of the MACF-1 homologue caused the phenotypic changes observed in our patient.

This is, to our knowledge, the first description of a disease related directly to the MACF1 gene. The muscle phenotype is really interesting considering that MACF1 display structural similarity to dystonin and dystrophin. Thus defects in MACF1 in general could be related to undiagnosed myopathic phenotypes.

57

The compact mutation of myostatin causes skeletal muscle hyperplasia

J. Baán, T. Kocsis, A. Keller-Pintér, L. Mendler, L. Dux

University of Szeged, Faculty of General Medicine, Institute of Biochemistry, Szeged, Hungary

Myostatin is an important negative regulator of skeletal muscle growth. Silencing myostatin gene in knockout mice causes hypermuscularity characterized by both muscle hyperplasia and hypertrophy. Compact ( Cmpt) mice carrying a 12-bp-deletion in the myostatin propeptide region show hypermuscularity as well. We aimed at analyzing the Cmpt phenotype to determine whether their muscles show either hyperplasia or hypertrophy or both.

We removed typical hindlimb muscles ( $m$. quadriceps, $m$. tibialis anterior, $\mathrm{m}$. extensor digitorum longus, $\mathrm{m}$. soleus, $\mathrm{m}$. gastrocnemius) from ten-week old male Cmpt mice as well as from control CFLP and $\mathrm{BALB} / \mathrm{c}$ strains. Tibialis anterior muscles were cryosectioned and stained by hematoxylin-eosin. Pictures from all microscopic fields of each muscle were reconstructed and the number and size of muscle fibers were analyzed by Digimizer software.

All muscles of the Cmpt mice were significantly larger compared to those of control ones. The number of muscle fibers in $\mathrm{m}$. tibialis anterior increased significantly $(5242 \pm 228$ vs. $3381 \pm 108$ or $3093 \pm 52$ (Cmpt vs. CFLP or BALB/c, mean \pm SEM)). Mean and frequency distribution of fiber size were similar in Cmpt and BALB/c mice $\left(2114 \pm 118 \mu \mathrm{m}^{2}\right.$ and $2018 \pm 75 \mu \mathrm{m}^{2}$ respectively). However, in CFLP control mice, the mean $\left(2527 \pm 122 \mu \mathrm{m}^{2}\right)$ and shape of fiber size distribution differed significantly from both of them. Thus, BALB/ $\mathrm{c}$ mice seem to be a better control than CFLP mice in this regard.

In contrast to myostatin knockout, hypermuscularity of Cmpt mice was caused exclusively by fiber hyperplasia which suggests a different mechanism of muscle overgrowth in the later strain.

\section{8}

The role of female sex hormones in aging related muscle weakness

\section{S. Sipilä, E. Pöllänen, V. Kovanen}

University of Jyväskylä, Gerontology Research Center, Department of Health Sciences, Finland

Previous data on women suggest decline in muscle performance around the age of 50 suggesting sex hormones as a mechanism for age-induced muscle weakness. We performed a RCT among 50-57year-old women including 1-year HRT and a case-control twin study with 54-62-year-old MZ pairs discordant for HRT.

Muscle power, thigh muscle cross-sectional area and composition were assessed and muscle samples taken. After 1-year of HRT, power increased on average $7 \%$ compared with $5 \%$ decline in the controls $(P=0.014)$. Muscle CSA increased $6 \%$ compared with $1 \%$ increase in the controls $(P<0.001)$ and the relative proportion of fat in the muscle decreased $5 \%$ after HRT vs. $17 \%$ increase in the controls $(P=0.009)$. The twin sisters on HRT had $16 \%$ greater power 
$(P=0.023)$ and $32 \%$ greater peak twitch torque $(P=0.002)$ than their co-twins. HRT using twin sisters had $6 \%$ greater muscle area $(P=0.065)$ and $5 \%$ lower relative fat area $(P=0.047)$ than their cotwins. In the RCT, global transcriptome analysis showed e.g. notable changes in genes related to proteolysis and peptidolysis among the controls. HRT up-regulated the expression of IGF-1 gene, especially MGF. HRT using twin sisters had up-regulated genes in processes related to cell structure regulation and down-regulated genes in processes related to e.g. cell-matrix interaction and energy metabolism.

Menopausal hormonal changes are among the key factors in the development of muscle weakness. HRT has beneficial effects on skeletal muscle among postmenopausal women. Given the known contraindications and potential harms related to the HRT, further development and studies are needed.

Supported by the EU Framework 7 Project MYOAGE: Understanding and Combating age related muscle weakness Grant Agreement Number: 223576.

59

Gender differences in muscle fiber characteristics after 8 weeks of resistance training

T. Moro ${ }^{1}$, F.Q. Pacelli ${ }^{1}$, L. Toniolo ${ }^{1}$, M. Canato ${ }^{1}$, P. Cancellara ${ }^{1}$, D. Miotti ${ }^{2}$, C. Reggiani ${ }^{1}$, A. Paoli ${ }^{1}$

${ }^{1}$ University of Padova, Dept. Biomedical Sciences, Padova, Italy; ${ }^{2}$ Maugeri Foundation, Pavia, Italy

Heavy resistance training (RT) promotes skeletal muscle hypertrophy decreasing MHC IIx and MHC I and increasing MHC IIa expression. But gender differences in these changes are not known. The aim of this study was to analyse the differences in gender response after two months of RT comparing the changes in muscle fibres characteristics with the mechanical measurement.

Eighteen healthy volunteers participated in 8-week progressive RT for upper limbs muscles. One repetition maximal (1RM) test and mechanical and myosin characterization of latissimus dorsi muscle fibres were analysed pre and post- training using a fine needle biopsy technique.

The increase in $1 \mathrm{RM}$ was significantly greater in women (+24\%) compared to men $(+13 \%)$. The electrophoretic analysis showed an increased in MHC IIa (male $+13 \%$ and female $+33 \%$ ) and a decrease in MHC IIx (male $-8 \%$ and female $-26 \%$ ) while the MHC I expression in male tends to decrease $(-5 \%)$ and in female tends to increase $(+6 \%)$. The two-way ANOVA analysis (time $x$ gender) showed a gender significant difference for female only in MHC IIa expression. Training increased significantly the cross sectional area (CSA) and fibre strength in male and muscle fibre tension in both groups.

The greater increase of 1RM performance with a substantial unchanged fibre CSA in female subjects could be explained by an improved motor units recruitment whilst males showed a greater hypertrophic response. Moreover also myosin changes showed a gender related difference. Taken together our results suggest that resistance training effects on muscle are gender specific.

Moro is a candidate for a young investigator award to be judged during the EMC 2012.

\section{4}

Separate control of oscillatory and isometric contraction in insect flight muscle

A. Katzemich ${ }^{1}$, C. Eldred ${ }^{2}$, F. Thiele ${ }^{1}$, K. Leonard ${ }^{3}$, J. Sparrow ${ }^{1}$, D. Swank ${ }^{2}$, B. Bullard ${ }^{1}$
${ }^{1}$ University of York, Dept of Biology, York, YO10 5DD, UK;

${ }^{2}$ Rensselaer Polytechnic Institute, Dept of Biology \& Center for Biotechnology and Interdisciplinary Studies, 110 8th Street, Troy, NY 12180, USA; ${ }^{3} \mathrm{EBI}$, Cambridge, CB10 1SD, UK

The indirect flight muscle (IFM) of insects has two modes of contraction regulated by different isoforms of $\mathrm{TnC}$. The troponin of Lethocerus IFM has TnT, TnH (the TnI of IFM) and TnC isofoms F1 and $\mathrm{F} 2$ in a ratio of 10:1. F1 binds a single calcium in the C-lobe and $\mathrm{F} 2$ binds one calcium in the $\mathrm{N}$ - and $\mathrm{C}$-lobes. $\mathrm{F} 1$ is needed for stretchactivated oscillatory contractions at a constant priming concentration of calcium. F2 is needed for isometric force and is regulated by exchangeable calcium in the $\mathrm{N}$-lobe. TnH binds to $\mathrm{F} 1$ solely at the $\mathrm{C}$-lobe and binding is independent of calcium. However, the N-lobe of $\mathrm{F} 1$ is necessary for stretch-activated contraction.

The C-terminal half of F1 (F1-Ct) when added to native fibres inhibited stretch-activated and isometric contraction, but fibres still developed isometric force with added F2. F1-Ct displaced F1 from fibres and this in turn was displaced by F2. Drosophila IFM has similar TnC isofoms. F1 and F2 were down-regulated by RNAi. Flies without F1 were flightless; the amount of F2 was doubled and IFM sarcomere structure was abnormal. Flies without F2 flew normally; F1 was increased by $10 \%$ and sarcomere structure was normal. Therefore, F1 alone can produce normal flight performance. Mechanical measurements with IFM from knock-down flies were compared with those from wild-type. In flies without F2, the calcium-sensitivity of work and power were reduced at intermediate calcium concentration and calcium-dependence was less co-operative. Therefore, F2 is needed to increase performance at moderate calcium concentration.

Katzemich is a candidate for a young investigator award to be judged during the EMC 2012.

\section{5}

Effect of the compact mutation on the glycogen distribution of skeletal muscle fibres

T. Kocsis, J. Baán, L. Mendler, A. Keller-Pintér, L. Dux

University of Szeged, Faculty of General Medicine, Institute of Biochemistry, Szeged, Hungary

Myostatin is a potent negative regulator of skeletal muscle mass. Compact (Cmpt) mice carry a naturally occurring 12-bp deletion in the propeptide region of the myostatin precursor. However, this increase in muscle mass is not accompanied by proportionate increase in muscle force. Our aim was to further characterize the phenotype of Cmpt mice and analyse the glycogen content and distribution of the muscles.

Frozen sections of tibialis anterior muscles of Cmpt, control CFLP and $\mathrm{BALB} / \mathrm{c}$ mice were stained by PAS to visualize glycogen content of the samples. The full cross sectional areas of the muscles were reconstructed from the microscopic photos by Cell B software, the size of the fibers was measured by Digimizer programme and the intensity of PAS staining was densitometrically analysed on greyscale images (0-1 OD). Body weight and the weight of hindlimb muscles of Cmpts significantly increased, while those of investigated organs (heart, liver, kidney) significantly decreased compared to both controls. In the fibers of tibialis anterior muscle of the Cmpt mice the average intensity of PAS staining was lower, the glycogen content per fibre significantly decreased compared to CFLPs. The frequency distribution of fibres with different glycogen content was narrower and shifted towards fibers with low glycogen content in both Cmpt and $\mathrm{BALB} / \mathrm{c}$ compared to CFLP.

Based on our results the lower glycogen content of Cmpt fibers compared to both controls might contribute to the lack of proportionate muscle force increase observed previously.

This work was supported by TÁMOP-4.2.2/B-10/1-2010-0012. 


\section{6}

Age-related differences in achilles tendon properties and triceps surae muscle architecture in vivo

\section{Stenroth ${ }^{1,2}$, J. Peltonen ${ }^{2}$, S. Sipilä ${ }^{1}$, T. Finni ${ }^{2}$}

${ }^{1}$ Gerontology Research Centre, Department of Health Sciences, University of Jyväskylä, Finland; ${ }^{2}$ Neuromuscular Research Center, Department of Biology of Physical Activity, University of Jyväskylä, Finland

Changes in tendon properties and in muscle architecture with aging can influence muscle function. Aim of the study was to identify agerelated differences in Achilles tendon properties and triceps surae muscle architecture with a comprehensive sample of young and old people.

A total of 100 subjects [33 young $(24 \pm 2$ year) and 67 old (75 \pm 3 year)] participated in this study. Motion analysis assisted ultrasonography was used to determine Achilles tendon stiffness, Young's modulus and hysteresis during isometric ramp contractions. Ultrasonography was used to measure muscle architectural features from triceps surae muscles, Achilles tendon thickness and cross-sectional area. Older participants had $17 \%(P<0.001)$ lower Achilles tendon stiffness and $32 \%(P<0.001)$ lower Young's modulus compared to young participants. Achilles tendon stiffness and Young's modulus were found to be associated with the triceps surae muscles force producing capacity $(r=0.580, P<0.001$ and $r=0.561$, $P<0.001$ for stiffness and Young's modulus, respectively) in combined data of young and old subjects. Triceps surae muscle size was smaller in old compared to young subjects, as indicated by $15 \%$ smaller anatomical cross-sectional area of gastrocnemius $(P<0.001)$ and smaller soleus $(P<0.05)$ and gastrocnemius medialis $(P<$ 0.001) muscle thickness, but Achilles tendon cross-sectional area was $20 \%$ larger $(P<0.001, n=71)$. A difference in muscle architecture between old and young subjects was observed only in gastrocnemius medialis muscle fascicle length $(P<0.05)$.

The results suggest that Achilles tendon mechanical properties adapt to match the level of muscle performance. Lower Achilles tendon stiffness could compensate for age-related decline in muscle function in locomotion.

Supported by the EU Framework 7 Project MYOAGE: Understanding and Combating age related muscle weakness Grant Agreement Number: 223576.

Stenroth is a candidate for a young investigator award to be judged during the EMC 2012.

\section{9}

\section{Structure and function of biceps and quadriceps muscles} in elderly subjects

P. Cancellara, M. Canato, T. Moro, A. Paoli, C. Reggiani, L. Toniolo University of Padua, Department of Biomedical Sciences, Padova, Italy

Aging and disuse are the two main conditions leading to skeletal muscle atrophy in humans. The age-related fibre atrophy is heterogeneous, and also affects different fibre types differently.

In this study we analysed skeletal muscle needle biopsies of elderly subjects (age 90-100), divided in 2 groups, one group still able to walk (W) and the other unable to walk (NW); two muscle biopsies (from biceps and quadriceps) were collected for each subject. The aim of this work was to compare muscles at single muscle fibre level, both in size and in mechanical performance. The following parameters were determined in single muscle fibres: cross sectional area (CSA), isometric force (Fo) and tension (Po), and Myosin Heavy Chain isoform composition.
The results obtained showed that: (1) CSA was greater in W group than in NW for both biceps and quadriceps muscles. Comparing different fibre types, the difference was more pronounced in $2 \mathrm{~A}$ fibres, (2) there was no statistically significant difference in isometric tension (Po) between $\mathrm{W}$ and NW group in both muscle types, (3) percentage distribution of fibre types pointed to a decrease in slow fibres in NW compared with $\mathrm{W}$ group in both muscles.

Ours results indicate that fibre types respond differently to disuse age-related effects. It is, therefore, important to assess fibre type distribution in skeletal muscles, in addition to studying whole muscles.

Supported by the EU Framework 7 Project MYOAGE: Understanding and Combating age related muscle weakness Grant Agreement Number: 223576.

Cancellara is a candidate for a young investigator award, to be judged during the EMC 2012.

\section{0}

\section{Role of Bmal1 gene in skeletal muscle}

M.I. Peña $\mathrm{Paz}^{1,2}$, K.D. Dyar ${ }^{1}$, B. Blaauw ${ }^{1,3}$, S. Ciciliot ${ }^{1,3}$, L. Agatea ${ }^{1}$, S. Schiaffino ${ }^{1}$

${ }^{1}$ Venetian Institute of Molecular Medicine (VIMM) Via Orus 2, 35129 Padua, Italy; ${ }^{2}$ University of Padua, Department of Biomedical Sciences, Via Ugo Bassi 58/b 35121 Padua, Italy; ${ }^{3}$ University of Padua, Department of Physiology and Anatomy, Via Marzolo 3, 35131 Padua, Italy

Circadian rhythms are responsible for various rhythmic 24-hour changes in physiological and behavioural parameters. A core oscillator located in the suprachiasmatic nucleus $(\mathrm{SCN})$ of the hypothalamus coordinates them. The SCN controls the endogenous timing system by coordinating the tissue-specific clocks present in all cells of the body. The regulation is based on a transcriptional-translational feedback loop that keeps internal gene expression entrained to the external light-dark cycle. The transcription factor Bmall is a major component of both central and peripheral clocks, and its absence leads to disruption of the 24-hour cycle.

In order to understand the function of the intrinsic muscle clock we are comparing a muscle specific knockout of the Bmall gene (BmallmKO) and their normal wildtype littermates, trying to elucidate the physiological relevance of the local clock function and its role in muscle activity, performance and metabolism.

We have observed that BmallmKO mice appear to have a normal lifespan and growth, yet with a small but significant increase in muscle mass. This hypertrophic phenotype is not accompanied by an increase in muscle force, and surprisingly there is a marked reduction in normalized force. Myofibrillar architecture is conserved in knockout muscles, and there are no major histological abnormalities in the muscles, yet myosin heavy chain composition is slightly shifted to fast type. We have been comparing the muscle circadian gene expression profile of these mice and their control littermates. Our preliminary analyses indicate that the transcription of many circadian muscle genes is greatly disrupted.

Peña Paz is a candidate for a young investigator award to be judged during the EMC 2012.

72

Colony filtration blot as a screening method for identification of soluble recombinant muscle protein expression in $E$. Coli

D. Müllerova, M. Ignatushchenko, D. Martinez Molina, M. Dollhopf, P. Nordlund 
Evitraproteoma AB, Box 23052, 10435 Stockholm, Sweden (www.evitra.se)

The implementation of generic and efficient technologies for the production of recombinant proteins still remains a crucial step in academic structural and functional genomics programs, as well as in industry.

Evitra $\mathrm{AB}$ has developed a method for rapid colony screening for soluble protein expression in Escherichia coli, the colony filtration blot (CoFi-blot). The screen is based on separation of soluble protein from inclusion bodies by a filtration step on colony level. CoFi-blot is well suited for screening the libraries of $\mathrm{C}$ - or N-terminal deletions, two-way deletions and random mutations, and tens of thousands of variants can be screened in a single experiment. It has also been successfully used in other applications such as on libraries of membrane proteins, pooled expression libraries (e.g. pools of genes/ orthologes, ORFeoms) and for screening of soluble constructs stable under certain solubilisation conditions.

Within the MUZIC project, CoFi blot screening was used on truncation variants of a group of muscle Z-disk proteins which were previously found difficult to express in soluble state. The method also reflects the situation at scale-up since detection is performed on lysate and no fusion protein is required. In addition, it potentially increases the probability of obtaining well-diffracting crystals through its capacity to identify many different protein constructs in one single experiment.

73

Functional analysis of the E56G mutation of essential ventricular myosin light chain linked to hypertrophic cardiomyopathy using a transgenic mouse model

J. Lossie ${ }^{1}$, M. Taube ${ }^{1}$, D. Petzhold ${ }^{1}$, C. Köhnke ${ }^{1}$, H. Haase ${ }^{1}$, I. Morano ${ }^{1,2}$

${ }^{1}$ Max-Delbrück-Center for Molecular Medicine, Robert-Rössle-Str. 10, Berlin, Germany; ${ }^{2}$ University Medicine Berlin Charité, Berlin, Germany

Eight missense mutations of human essential ventricular myosin light chain (hVLC-1) linked to familial hypertrophic cardiomyopathy have yet been described. Recently, we showed that mutated hVLC-1 binds significantly weaker (with a $2-3$ fold higher $K_{\mathrm{D}}$ ) to the myosin lever arm than does normal hVLC-1. In particular, the E56G (hVLC-1 ${ }^{\mathrm{E} 6 \mathrm{G}}$ ) mutation revealed a 30 fold reduced binding affinity. We, therefore investigated the functional consequences of E56G mutated hVLC-1 $\left(\mathrm{hVLC}-1^{\mathrm{E} 56 \mathrm{G}}\right)$ on cardiac structure and performance using a transgenic mouse model.

We generated transgenic mice (TGM) that overexpressed normal hVLC-1 (TGM/hVLC-1) or hVLC-1 ${ }^{\text {E56G }}$ (TGM/hVLC- ${ }^{\text {E56G }}$ ) in cardiomyocytes using a mouse $\alpha$-myosin heavy chain promoter. Transgene expression of the heart was evaluated by RT-PCR, SDSPAGE, and Western-Blot analysis. All TGM lines revealed a normal heart-to-body-weigth ratio, and no obvious behavioral or physiological abnormalities. Cardiac structures and functions were investigated in vivo by echocardiography. Intrinsic cardiac contractile performance was monitored in vitro with isolated perfused hearts (Langendorff mode) and single cardiomyocyte contraction analysis. $\mathrm{TGM} / \mathrm{hVLC}-1$ and TGM/hVLC-1 ${ }^{\mathrm{E} 56 \mathrm{G}}$ expressed equal amounts of transgene, i.e. $45 \%$ hVLC-1 and $43 \%$ hVLC-1 ${ }^{\mathrm{E} 56 \mathrm{G}}$, respectively (\% of total essential myosin light chain of cardiac myofibrils), Interventricular septum and left ventricular posterior wall of TGM/hVLC$1^{\mathrm{E} 56 \mathrm{G}}$ were significantly thicker compared with age- and sex-matched (12 weeks old male mice) TGM/hVLC-1. Left ventricular pressure development and maximal relaxation rate of isolated perfused hearts prepared from TGM/hVLC- ${ }^{\mathrm{E} 56 \mathrm{G}}$ were significantly decreased compared with normal TGM/hVLC-1.
Depressed myosin binding affinity of hVLC-1 ${ }^{\mathrm{E} 56 \mathrm{G}}$ coincides with reduced intrinsic myocardial contractile functions and early onset of hypertrophy of distinct cardiac structures.

\section{4}

A mechanistic model of ca-activation of the thin filaments in cardiac muscle

N.A. Metalnikova, A.K. Tsaturyan

Institute of Mechanics, Department of Biomechanics, Moscow University, Moscow, Russia

Although cardiac $\mathrm{TnC}$ binds only one $\mathrm{Ca}^{2+}$ ion, the force-calcium curve is very steep. Blocking the actomyosin interaction with blebbistatin does no decrease the slope indicating that the cooperativity is an intrinsic property of the thin filaments. The presence of force-generating myosin heads increases Ca-sensitivity not affecting the slope. In rigor the system becomes very sensitive to $\mathrm{Ca}^{2+}$, but not cooperative (Sun, Irving, J. Physiol. 2009). All these features lack quantitative explanation.

We propose a mechanistic model where following Smith (J. Phys. A. 2001) tropomyosin is considered as a continuous flexible chain in a harmonic potential well on the actin helix. The chain can be pinned to actin by TnI (B-state) or (in opposite direction) by strongly bound myosin heads (O-state). Mathematical formalism expands that of Smith (2001), Smith and Geeves (Biophys. J., 2003) by assuming that tropomyosin can be stretched or compressed by an external force and by using a three-pin approximation of tropomyosin chain. A MonteCarlo simulation of binding-unbinding events was performed for a thin filament model consisting of 183 actins and 27 troponins. Up to $10^{7}$ iterations for each myosin head and TnI were performed at each calcium concentration. With proper parameters the model predicts calcium curve with the Hill coefficient, $n$, of 2.5 even in the absence of myosin heads. Myosin binding increases Ca-sensitivity, not affecting the steepness of the curve. In rigor $n$ reduces to 1 while the calcium sensitivity increases dramatically. These modeling results reproduce well some experimental data.

Supported by RFBR.

\section{6}

The impact of experimentaly induced ischemia reperfussion upon physiological parameters and ultrastructure in aging rat heart

F. Revnic ${ }^{1}$, A.S. Nica ${ }^{2}$, C. Revnic, B. Paltineanu ${ }^{1}$, G. Dinu ${ }^{3}$, G. Prada ${ }^{2}$, C. Pena ${ }^{1}$, S. Prada ${ }^{1}$, C.R. Revnic ${ }^{4}$

${ }^{1}$ Biology of Aging, NIGG "Ana Aslan", Bucharest, Romania; ${ }^{2}$ UMF “Carol Davila”, Bucharest, Romania; ${ }^{3}$ Floreasca Emergency Hospital, Bucharest Romania; ${ }^{4}$ Ambroise Pare ${ }^{\text {Hospital, }}$

University of Medicine, Paris, France

The aim of our study was to point the effect of $45 \mathrm{~min}$ ischemia followed by varying periods of reperfusion on isolated rat heart of different ages upon physiological parameters of cardiac contractility, to assay ventricular cells for apoptosis and to investigate the ultrastructural changes in heart muscle.

Isolated hearts of 6 and 24 month old rats have been mounted and perfused with Krebs-Hanseleit buffer at $24 \mathrm{C}$ in Langerdoff retrograde perfusion system at a constant pressure over $30 \mathrm{~min}$ (stabilization period followed by $45 \mathrm{~min}$ ischemia and then reperfused for $60 \mathrm{~min}$ (i.e at $\left.10^{\prime}, 20^{\prime}, 30^{\prime}, 40^{\prime}, 50^{\prime}, 60\right)$. We determined: heart rate, (H.R), coronary flow $(\mathrm{C}$ F.) and LVPD (left ventricle pressure developed). TACS Apoptotic DNA Laddering kit has been used to assay heart cells for apoptosis after $60 \mathrm{~min}$ reperfusion. 
In old rats, cardiac frequency exhibited higher values than in control. Coronary flow was variable in time in aging rats in comparison with young ones where a slow decrease was observed during the experiment. Pressure developed by the left ventricle was net elevated in aging rats but with fluctuations in time. No internucleosomal fragmentation and DNA laddering displaying has been observed for this experimental model. Our electron microscopy studies have pointed out greater damage in old hearts in terms of myofiber tears, mitochondrial and sarcoplasmic reticulum disruption.

Our data have pointed out that aging rat heart has an increased reactivity towards stress conditions imposed by ischemia-reperfusion.

77

Insights for the release of phosphate by myosin motors

P. Llinas ${ }^{1 *}$, T. Isabet ${ }^{1 *}$, L. Song ${ }^{2 *}$, A. Zong ${ }^{2}$, H.L. Sweeney ${ }^{2}$, A. Houdusse ${ }^{1}$

${ }^{1}$ Institut Curie CNRS, Structural Motility, UMR144, Paris, France; ${ }^{2}$ University of Pennsylvania, School of Medicine, Department of Physiology, Philadelphia, PA, USA *These co-authors have contributed equally to this work.

Force production in myosin motors is tightly coupled to transitions promoted by actin binding that allow release of phosphate followed by release of ADP. While the release of phosphate is the rate limiting step for conventional muscle myosin II, it is much faster for several unconventional myosins. Coupling structural and kinetic studies for different myosins has shown that they populate similar structural states. These studies have elucidated how certain transitions of the actin-myosin catalytic cycle, including the ATP-induced dissociation of myosin from actin and for the ATP hydrolysis step that allows priming of the lever arm. Not yet visualized however are the structural rearrangements triggered by actin binding that are coupled to force generation and product release.

While phosphate is produced in the pre-powerstroke (ADP.Pi) state, it is also trapped in this state until an actin-activated transition opens a back-door to promote its release from the active site. It is unclear, however, which of the three elements of the active site (Switch I, Switch II and the P-loop) play a role in this transition and whether the major cleft within the motor partially closes to allow this release while keeping the lever arm primed.

We have crystallized a new structural state for myosin VI in the presence of MgADP and phosphate that has an open back-door due to a Switch II rearrangement and a lever arm primed. Thus it has the expected features of the previously unseen Pi release state of myosin. Kinetic and structural studies on a number of mutants from different myosins are currently being performed to evaluate this possibility. The structure is consistent with cleft closure occurring subsequent to Pi release, which is also being tested. An interesting finding in this new structure is that the SH1 helix kinks to allow the converter to stay in the primed position, the signature of a state at the beginning of force production.

\section{8}

Structural properties of actin-myosin complexes in contracting rabbit muscle fibres subjected to stretch or producing mechanical work

A.K. Tsaturyan ${ }^{1}$, N.A. Koubassova ${ }^{1}$, S.Y. Bershitsky ${ }^{2}$, M. Fernandez ${ }^{3}$, O. Narayanan ${ }^{3}$, M.A. Ferenczi ${ }^{4}$

${ }^{1}$ Moscow University, Institute of Mechanics, Department of Biomechanics, Moscow, Russia; ${ }^{2}$ Institute of Immunology and
Physiology, Yekaterinburg, Russia; ${ }^{3}$ European Synchrotron Radiation Facility B.P. 220, beamline ID02, Grenoble, France; ${ }^{4}$ Imperial College London, Molecular Medicine Section, National Heart and Lung Institute, London, United Kingdom

Low angle time-resolved x-ray diffraction (beamline ID02, ESRF, Grenoble, France) was used to study structural properties of crossbridges in permeabilized fibres of rabbit skeletal muscle at full activation at near physiological temperature of $31-34{ }^{\circ} \mathrm{C}$.

Bundles of 2-3 fibres were stretched at a constant velocity of $1-1.2$ length/s or allowed to shorten at a load of ca. $P_{0} / 3$ or $2 P_{0} / 3$ where $P_{0}$ is isometric tension. Two $10-30 \mathrm{~ms}$ long x-ray frames were taken in each run of a protocol: during isometric contraction and at the end of the ramp stretch or shortening. The two $2 \mathrm{D}$ x-ray diffraction patterns were compared. Changes in fibre stiffness were measures in control experiments.

Stretch induced a decrease in the meridional M3 intensity and in the off-meridional intensities of M1, M2 myosin and A1, A2 actin layer lines and to a $\sim 30 \%$ increase in stiffness. The data indicate that stretch unlocks stereo-specifically bound myosin heads and promotes non-stereo-specific binding to actin of detached myosin explaining high stiffness and low ATPase rate of contracting muscle during stretch. Shortening decreases stiffness, the intensities of M3, actin layer lines and of the $\mathrm{AM}_{+1}$ actin-myosin layer line at $\sim 10.3 \mathrm{~nm}$. The intensity decrease was very small during slow shortening and more pronounced under load below $0.4 P_{0}$.

The data show that three processes: 'roll and lock' transition of actin-myosin complexes, tilt of the myosin lever arm and the rate of detachment of strongly bound myosin heads have different straindependence.

Supported by the Royal Society, EMBL, RFBR and RAS.

\section{9}

Modified EC coupling in myostatin deficient $\left(\mathrm{MSTN}^{-/-}\right)$mice

A. Jenes ${ }^{1}$, D. Bodnár ${ }^{1}$, O. Ruzsnavszky ${ }^{1}$, N. Geyer ${ }^{1}$, B. Dienes ${ }^{1}$, A. Balogh ${ }^{2}$, Z. Papp ${ }^{2}$, P. Szentesi ${ }^{1}$, L. Csernoch ${ }^{1}$

${ }^{1}$ University of Debrecen, Medical and Health Science Center, Department of Physiology, Debrecen, Hungary; ${ }^{2}$ University of Debrecen, Medical and Health Science Center, Department of Cardiology, Debrecen, Hungary

Myostatin, a member of the transforming growth factor $\beta$ superfamily has emerged as a potent negative regulator of skeletal muscle growth. During embryogenesis, myostatin influences the differentiation and proliferation of myoblasts. $\mathrm{MSTN}^{-/-}$mice have increased muscle mass demonstrating that myostatin is a negative regulator of skeletal muscle development. Excessive muscle mass in $\mathrm{MSTN}^{-1-}$ mice is associated with a profound loss of oxidative metabolic properties.

In our studies we compared MSTN $^{-1-}$ and control C57/BL6 mice. We investigated the muscle performance during moderate intensity voluntary wheel running and in grip tests. We measured force and fatigue of musculus soleus (SOL) and extensor digitorum longus (EDL). Isometric force measurements were performed during repeated activation-relaxation cycles. $\mathrm{pCa}$-force relations were determined to assess the $\mathrm{Ca}^{2+}$-sensitivity of force production. Changes in intracellular $\mathrm{Ca}^{2+}$ concentration were measured in single fibers.

In voluntary wheel running $\mathrm{MSTN}^{-1-}$ mice performed better on average $(10.8 \pm 0.8$ versus $7.9 \pm 1.0 \mathrm{~m} / \mathrm{min})$, in maximal speed $(25.4 \pm 1.3$ versus $16.8 \pm 1.3 \mathrm{~m} / \mathrm{min})$, and in total distance $(7.50 \pm 0.88$ versus $5.57 \pm 0.53 \mathrm{~km} /$ day), but they spent less time in the wheel $(585 \pm 59$ versus $622 \pm 40 \mathrm{~min} /$ day $)$. In grip tests $\mathrm{MSTN}^{-1-}$ mice showed higher average force $(0.24 \pm 0.01$ versus $0.13 \pm 0.01 \mathrm{mN})$. Single twitches in EDL $(102.8 \pm 30.0$ vs $84.5 \pm 43.9 \mathrm{mN})$ and SOL $(138.3 \pm 45.7$ vs $49.1 \pm 24.8 \mathrm{mN})$ were 
stronger in the control strain. pCa-force relations and calciumtransients evoked by $\mathrm{KCl}$ depolarization did not differ. Resting intracellular calcium concentrations were significantly elevated in $\mathrm{MSTN}^{-1-}$ mice $(464 \pm 159$ versus $573 \pm 123 \mathrm{nM})$.

Our results from in vitro experiments do not explain our in vivo findings.

\section{0}

Altered cross bridge kinetics in skeletal myofibrils from neb $\Delta E X 55$, a novel mouse model of nebulin-based nemaline myopathy

C. Ferrara ${ }^{1}$, C. Buck ${ }^{2}$, N. Piroddi ${ }^{1}$, C. Tesi ${ }^{1}$, J. LeWinter ${ }^{3}$, C. Ottenheijm ${ }^{2,3}$, C. Poggesi ${ }^{1}$, H. Granzier ${ }^{3}$

${ }^{1}$ Università di Firenze, Dipartimento di Scienze Fisiologiche, Firenze, Italy; ${ }^{2}$ VU University Medical Center, Department of Physiology, Amsterdam, The Netherlands; ${ }^{3}$ University of Arizona, Department of Physiology, Tucson, AZ, USA

Nemaline Myopathy is the most common non-dystrophic congenital myopathy, clinically characterized by muscle weakness. The disease is most often associated with mutations in the nebulin gene and the nebulin-based disease is referred to as NEM2. Recent work on skinned muscle fibres from NEM2 patients revealed remarkable phenotypic similarities to fibres from nebulin $\mathrm{KO}$ mice (Ottenheijm et al. Frontiers in Physiology, 2012). Here we investigated mechanics and kinetics of single myofibrils from a novel NEM2 mouse model (NEB $\Delta$ ex55) that mimics a deletion in the nebulin gene found in a large group of NEM2 patients.

We used rapid solution switching (Tesi et al., Biophys. J., 2002) to compare maximal tension and kinetics of contraction and relaxation of myofibrils isolated from frozen skeletal muscles (tibialis cranialis of neonatal mice, $\sim 7$ days) of WT and NEB $\Delta$ ex 55 mice. Myofibrils, mounted in a force recording apparatus $\left(15^{\circ} \mathrm{C}\right)$, were maximally $\mathrm{Ca}^{2+}$-activated ( $\mathrm{pCa} 4.5$ ) and fully relaxed ( $\mathrm{pCa} 9.0$ ).

Maximal isometric tension was markedly reduced in NEB $\Delta$ ex55 mouse myofibrils $\left(49.7 \pm 10.6 \mathrm{mN} \mathrm{mm}{ }^{-2} n=11\right)$ compared to WT $\left(135.3 \pm 16.9 \mathrm{mN} \mathrm{mm}^{-2} n=9\right)$. The rate constant of active tension generation following maximal $\mathrm{Ca}^{2+}$ activation $\left(k_{\mathrm{ACT}}\right)$ was significantly reduced in myofibrils from the NEB $\Delta$ ex55 mouse model $\left(1.46 \pm 0.07 \mathrm{~s}^{-1}\right)$ compared to WT $\left(2.75 \pm 0.27 \mathrm{~s}^{-1}\right)$. Force relaxation kinetics upon $\mathrm{Ca}^{2+}$ removal were remarkably faster in NEB $\Delta$ ex55 mouse myofibrils than in WT, evidence that the apparent rate with which cross-bridges leave the force generating states is accelerated in the NEB $\triangle$ ex 55 sarcomeres.

Reduction in the rate with which cross-bridges enter the force generating state and increase in the rate of cross bridge dissociation can markedly contribute to reducing maximal tension and are expected to increase the energetic cost of tension generation of the NEB $\Delta$ ex55sarcomeres. The results suggest that nebulin plays a significant role in contraction regulation and that altered cross bridge kinetics contribute to NEM2 pathogenesis.

\section{2}

\section{p42/44 MAPK signaling mediates MYPT1 isoform expression}

\section{N.J. Brozovich, G.P. Hawes, S. Yuen, O. Ogut, F.V. Brozovich}

Mayo Medical School, Cardiovascular Diseases, Rochester, MN 55902 USA

During NO mediated vasodilatation PKG activates myosin light chain (MLC) phosphatase, which dephosphorylates the MLC to produce smooth muscle relaxation. MLC phosphatase is a trimeric enzyme consisting of a catalytic, myosin targeting (MYPT1) and $20 \mathrm{kDa}$ subunits. Alternative mRNA splicing produces four MYPT1 isoforms, which differ by the presence or absence of central insert (CI) and a carboxy-terminal luecine zipper (LZ). PKG mediated activation of MLC phosphatase requires the expression of a LZ+ MYPT1 isoform, and thus, the sensitivity to $\mathrm{NO}$ mediated vasodilatation is mediated, in part, by LZ+/LZ- MYPT1 expression. We have reported that during heart failure, the increase in AngII is associated with an activation of p42/44 MAPK signaling and a decrease in LZ+ MYPT1 expression.

The mechanism for angiotensin induced regulation of LZ+ MYPT1 expression was assessed in cultured vascular smooth muscle cells (SMCs). For these experiments, LZ+ MYPT1 expression was determined in cultured SMCs at $24 \mathrm{~h}, 48 \mathrm{~h}$ and $72 \mathrm{~h}$ after Ang II treatment $(1 \mu \mathrm{M})$ and compared to the control of time in culture alone. To investigate if the activation of p42/44 MAPK mediates the AngII induced decrease in LZ+ MYPT1 expression, cultured SMCs were stimulated with AngII in SMCs pretreated with a MEK inhibitor (20 $\mu \mathrm{M}$ PD98059), and the time course of p42/44 MAPK activation as well as LZ+ MYPT1 expression was determined.

AngII resulted in a time dependent activation of p42/44 MAPK. Compared to control SMCs, SMCs treated with AngII had a significant decrease in LZ+ MYPT1 expression. However pretreatment of the SMCs with a MEK inhibitor (PD98059) blocked the AngII induced activation of p42/44 MAPK and decrease of LZ+ MYPT1 expression.

These results demonstrate that LZ+ MYPT1 expression is regulated by a p42/44 MAPK signaling pathway. During heart failure, treatment with either ACE inhibitors or AngII receptor blockers will prevent the activation of $\mathrm{p} 42 / 44$ MAPK and the resulting decrease in LZ+ MYPT1 expression to preserve the sensitivity of vascular smooth muscle to NO mediated vasodilatation.

\section{6}

\section{The super relaxed state in diverse muscle types}

\section{E. Pate ${ }^{1}$, N. Naber ${ }^{2}$, R. Cooke ${ }^{2}$}

${ }^{1}$ Washington State University, Dept of Bioengineering, Pullman WA, USA; ${ }^{2}$ University of California, Dept of Biochemistry, San Francisco, CA, USA

Recently, we observed a new state of skeletal myosin, the super relaxed state (SRX), with a very slow ATP turnover rate. Inhibition of the myosin ATPase activity in the SRX was suggested to be caused by the binding of the myosin head to the core of the thick filament in a structural motif identified earlier by electron microscopy.

The SRX also occurs in cardiac muscle. In both skeletal and cardiac muscle the SRX appears to play a similar role in relaxed cells, providing a state with a very low metabolic rate. However, in active muscle the properties of the SRX differ dramatically. We observed a rapid transition of myosin heads out of the SRX in active skeletal fibers, while the population of the SRX remained constant in active cardiac cells. This property allows the SRX to play a very different role in cardiac muscle, regulating the active force generated by cross-bridges and implying that at the level of the actomyosin interaction, cardiac muscle is regulated at both the thick- and thinfilament levels.

We have recently extended our approach to tarantula leg muscle. We find a SRX, with a very slow nucleotide turnover time constant, $\sim 30$ min. Similar to cardiac muscle, myosin in tarantula fibers can remain in the SRX upon activation, suggesting a role in the regulation of active force. The fact that two such evolutionarily distant muscles, vertebrate cardiac and arachnid exoskeletal, both display thickfilament regulation of active force involving the SRX suggests this mechanism of regulation can be anticipated to be widely distributed among muscle types. 
87

Impact of site-specific phosphorylation of the protein kinase $\mathbf{C}$ sites Ser42/44, Thr143 and Ser198 of cardiac troponin $i$ on calcium sensitivity in human cardiomyocytes

P.J.M. Wijnker ${ }^{1}$, D.B. Foster ${ }^{2}$, A.M. Murphy ${ }^{2}$, G.J.M. Stienen ${ }^{1,3}$, J. van der Velden ${ }^{1}$

${ }^{1}$ VU University Medical Center, Institute for Cardiovascular Research, Laboratory for Physiology, Amsterdam, The Netherlands; ${ }^{2}$ Johns Hopkins University School of Medicine, Department of Pediatrics/Division of Cardiology, Baltimore, USA; ${ }^{3}$ VU University Amsterdam, Department of Physics and Astronomy, Amsterdam, The Netherlands

Perturbations in protein kinase C (PKC) isoform expression and localization have been reported in failing cardiomyocytes. Previous studies suggested that PKC-mediated phosphorylation of troponin I (cTnI) underlies myofilament dysfunction in cardiac disease. PKC phosphorylates cTnI at various sites. The functional effects of PKCmediated cTnI phosphorylation at these different sites in human myocardium are unknown. In the present study, we investigated the effects of cTnI phosphorylation at well-known PKC-sites Ser42/44 and Thr143, and the recently identified PKC-site Ser198 on myofilament $\mathrm{Ca}^{2+}$-sensitivity $\left(\mathrm{pCa}_{50}\right)$ in human failing cardiomyocytes.

Myofilament force development was measured at various $\left[\mathrm{Ca}^{2+}\right]$ in permeabilized cardiomyocytes in which endogenous troponin complex was partially exchanged $(70.0 \pm 1.8 \%)$ with recombinant whole human troponin complexes. Site- directed mutagenesis was used to replace the Ser/Thr of the PKC-sites by aspartic acid (D) or alanine (A) to mimic phosphorylation and dephosphorylation, respectivelyMyofilament $\mathrm{Ca}^{2+}$-sensitivity was significantly reduced by pseudophosphorylation of Ser42/44, while an increase was found with pseudophosphorylated Thr143 and Ser198 (see Figure).

\section{Calcium sensitivity}

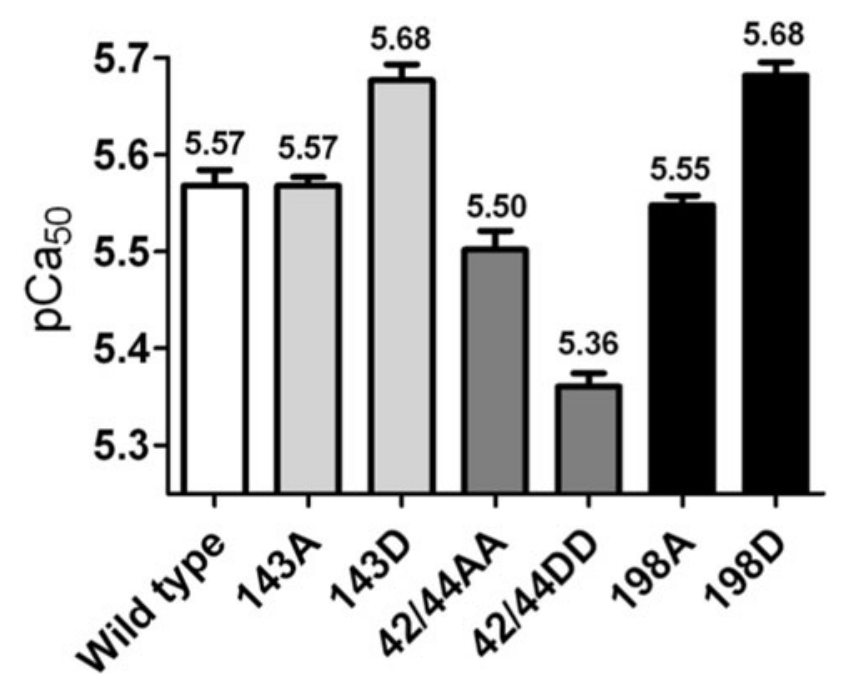

These opposite site-specific effects suggest that the collective effect of various PKC isoforms on myofilament $\mathrm{Ca}^{2+}$-sensitivity depends on the degree at which the different sites are phosphorylated.

Wijnker is a candidate for a young investigator award to be judged during the EMC 2012.
91

Increased tension cost in human fhe with a sarcomere gene mutation

E.R. Paalberends ${ }^{1}$, N. Vazirpanah ${ }^{1}$, N. Boontje ${ }^{1}$, M. van Slegtenhorst $^{2}$, C. dos Remedios ${ }^{3}$, F.J. ten Cate $^{2}$, G.J.M. Stienen ${ }^{1,4}$, M. Michels ${ }^{2}$, J. van der Velden ${ }^{1}$

${ }^{1}$ Laboratory for Physiology, VU University Medical Center, Amsterdam, The Netherlands; ${ }^{2}$ Cardiology and Clinical Genetics, Thorax Center, Erasmus Medical Center, Rotterdam, The Netherlands; ${ }^{3}$ The University of Sydney, Institute of Biomedical Research, Muscle Research Unit, Sydney, Australia; ${ }^{4}$ Department of Physics and Astronomy, VU University Amsterdam, Amsterdam, The Netherlands.

Familial hypertrophic cardiomyopathy (FHC) is frequently caused by mutations in genes encoding sarcomeric proteins. Energy depletion of the heart is thought to initiate and promote FHC disease development. It has been hypothesized that FHC sarcomere gene mutations increase ATP utilization during sarcomere contraction and thereby increase energy demand of the heart. In the present study we investigated to what extent the economy of contraction differed between sarcomere mutation positive and sarcomere mutation negative FHC patients.

Maximal force generating capacity $\left(\mathrm{F}_{\max }\right)$ and ATP consumption $\left(\mathrm{ATP}_{\max }\right)$ were simultaneously measured in Triton-permeabilized left ventricular (LV) muscle strips of 13 patients with heterozygous mutations in myosin binding protein $\mathrm{C}\left(M Y B P C 3_{\text {mut }}\right), \beta$-myosin heavy chain $\left(M Y H 7_{\text {mut }}\right), \alpha$-tropomyosin $\left(T P M 1_{\text {mut }}\right)$, troponin I $\left(T N N I 3_{\mathrm{mut}}\right)$, and a homozygous mutation in troponin $\mathrm{T}\left(T N N T 2_{\mathrm{mut}}\right)$. $\mathrm{LV}$ strips of $5 \mathrm{FHC}$ sarcomere mutation negative patients $\left(\mathrm{FHC}_{\mathrm{smn}}\right)$ served as a control group. Economy of myofilament contraction is expressed as tension cost (TC), i.e. amount of ATP used during force development $\left(\mathrm{ATP}_{\max } / \mathrm{F}_{\max }\right)$.

TC was slightly higher in the heterozygous gene mutation groups and significantly higher in homozygous $T N N T 2_{\text {mut }}$ compared to $\mathrm{LVH}_{- \text {smn- }}$ (figure). TC was slightly higher in FHC with heterozygous mutations in thin filament proteins $\left(2.04 \pm 0.15 \mu \mathrm{mol} \mathrm{L}^{-1} \mathrm{~s}^{-1} /\right.$ $\left.\mathrm{kNm}^{-2}\right)$ compared to thick filament mutants $(1.84 \pm 0.08$ $\mu \mathrm{mol} \mathrm{L}-1 \mathrm{~s}^{-1} / \mathrm{kNm}^{-2}$ ).

\section{Tension cost in FHC}

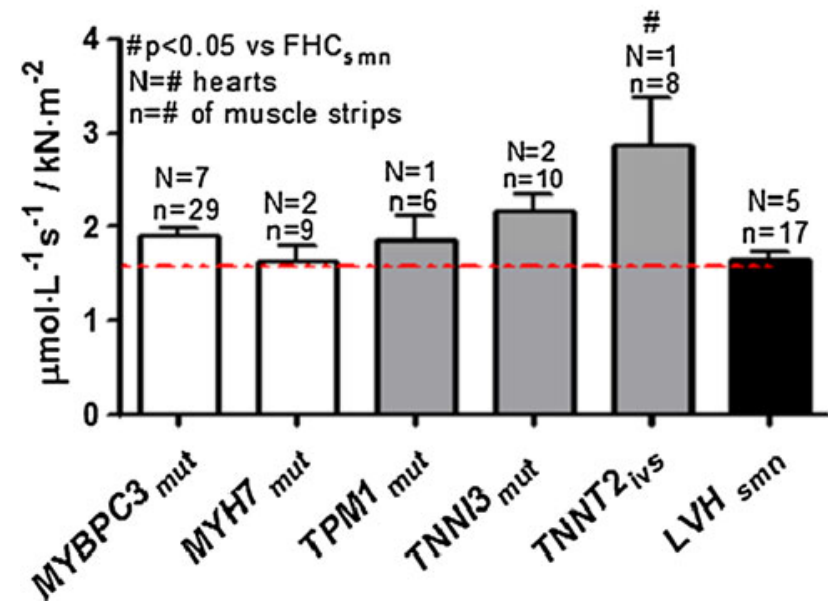

Overall, we observed a slight reduction in economy of contraction in $\mathrm{FHC}$ patients with a sarcomere gene mutation compared to $\mathrm{FHC}_{\mathrm{smn}}$ patients. The high TC value in the homozygous $T N N T_{\text {mut }}$ suggests that the mutant protein affects economy of sarcomere contraction in a dose-dependent manner. 
Paalberends is a candidate for a young investigator award to be judged during the EMC 2012.

\section{2}

\section{Autophagy inhibition induces functional impairment of mice fast} myosin isoform

M. Maffei ${ }^{1}$, S. Carnio ${ }^{2}$, E. Longa ${ }^{1}$, F. Lo Verso ${ }^{2}$, R. Bottinelli ${ }^{1}$, M. Sandri ${ }^{2}$, M. Canepari ${ }^{1}$

${ }^{1}$ University of Pavia, Department of Molecular Medicine and Interuniversity Institute of Myology, Via Forlanini 6, 27100 Pavia, Italy; ${ }^{2}$ Dulbecco Telethon Institute at Venetian Institute of Molecular Medicine, via Orus 2, 35129 Padova, Italy

Autophagy inhibition in knockout mice ( $\operatorname{Atg} 7$ null mice) induces myopathy and skeletal muscles functional impairment. Oxidative damage of contractile proteins could contribute to the functional impairment. The goal of this study was to assess the oxidative damage of actin and myosin from Atg7 null muscles and investigate their function at cellular and molecular level. The effect of an antioxidant drug Trolox was also investigated.

Gastrocnemius muscles of WT (control), KO (Atg7 knockout), TRO (control treated with Trolox for 3 weeks) and KOTRO (Atg7 knockout treated with Trolox for 3 weeks) mice were dissected and used for: 1) single fibers preparation for mechanical measurements 2) actin and myosin extraction for "in vitro motility" (IVMA) experiments and 3) Oxyblot analysis.

The results show a significant reduction of cross sectional area (CSA) specific force (Po/CSA) and unloaded velocity (Vo) in $\mathrm{KO}$ fibers and a significant reduction of sliding velocity $(V f)$ of WT, KO, TRO and KOTRO actins on KO myosin. The results also show significant oxidative modifications of both actin and myosin in $\mathrm{KO}$ muscles but not significant differences among WT, KO, TRO and KOTRO actins prepared for IVMA experiments. Trolox administration did not prevent muscle fibre atrophy, but successfully prevented myosin oxidation and impairment of functional properties.

The data provide experimental evidence that intrinsic myosin dysfunction occurs in $\mathrm{Atg} 7$ null mice and may be related to oxidative damage.

Supported by the EU Framework 7 Project MYOAGE: Understanding and Combating age related muscle weakness Grant Agreement Number: 223576.

\section{7}

\section{Roles of calcium and oxidation in muscle weakness}

G.D. Lamb, J.P. Mollica, T.L. Dutka, R.M. Murphy

La Trobe University, Department of Zoology, Melbourne, VIC 3086, Australia

Skeletal muscle weakness can arise from dysfunction of any of the steps in the excitation-contraction coupling sequence, though it most often appears to occur due to either i) disruption of the coupling between the voltage-sensors/dihydropyridine receptors (DHPRs) in the transverse tubular system and the $\mathrm{Ca}^{2+}$ release channels/ryanodine receptors (RyRs) in the sarcoplasmic reticulum (SR), or ii) alterations in maximum force and/or $\mathrm{Ca}^{2+}$-sensitivity of the contractile apparatus. Raising cytoplasmic $\left[\mathrm{Ca}^{2+}\right]$ above its normal operating range, either by excessive influx of extracellular $\mathrm{Ca}^{2+}$ or excessive release of SR $\mathrm{Ca}^{2+}$, disrupts coupling between the DHPRs and the RyRs by an enzymatic process possibly involving activation of $\mu$-calpain, resulting in proteolysis of a junctional protein and physical alteration of the triad junction. Reactive oxygen and nitrogen species may exacerbate such $\mathrm{Ca}^{2+}$-induced disruption of the coupling by acting on the RyR to increase $\mathrm{Ca}^{2+}$ leakage or on surface membrane proteins to increase
$\mathrm{Ca}^{2+}$ influx. Such reactive species also readily modify the properties of the contractile apparatus. In fast-twitch fibres, mild oxidative stress can result in a reversible increase in $\mathrm{Ca}^{2+}$-sensitivity by S-glutathionylation of troponin $\mathrm{I}\left(\mathrm{TnI}_{\mathrm{f}}\right)$, whereas S-nitrosylation of that same site produces a reversible decrease in $\mathrm{Ca}^{2+}$-sensitivity. Prolonged and excessive oxidation however leads to irreversible decreases in both maximum force and $\mathrm{Ca}^{2+}$-sensitivity, as well as loss of the ability to increase $\mathrm{Ca}^{2+}$-sensitivity by S-glutathionylation of $\mathrm{TnI}_{\mathrm{f}}$.

106

The sarcomeric z-disk protein myopodin: localization, dynamics and interactions in living cells

I.G. Reza-Mazar ${ }^{1}$, F. Dierck ${ }^{2}$, N. Frey ${ }^{2}$, P. Van der Ven ${ }^{1}$, G. Kirfel ${ }^{1}$, D. Fürst ${ }^{1}$

${ }^{1}$ University of Bonn, Department of Molecular Cell Biology, Institute for Cell Biology, Germany; ${ }^{2}$ University of Kiel, Department of Cardiology and Angiology, Germany

The protein myopodin (encoded by the gene SYNPO2; also called genethonin-2, synaptopodin-2 and fesselin) is widely expressed in striated and smooth muscle cells and interacts with filamentous actin, $\alpha$-actinin and filamin. Different isoforms result from alternative splicing and the predicted size of the corresponding proteins varies between $76 \mathrm{kDa}$ and $136 \mathrm{kDa}$. The lower molecular mass myopodin isoforms were shown to be dual compartment proteins that redistribute between the nucleus and the cytoplasm in a differentiation-dependent and stressinduced manner. In addition myopodin displays actin-bundling activity.

Isolation of neonatal rat ventricular cardiomyocytes, Immunofluorescence staining, Fluorescence recovery after photobleaching and analysis of FRAP data and Caffeine and BDM treatment.

We examined the dynamic behaviour of EGFP-fusion proteins of the different myopodin-isoforms in neonatal rat ventricular cardiomyocytes (NRVC) upon adenoviral transduction. Immunofluorescence microscopy was used to confirm Z-disk localization of the proteins and to analyze the effects of their expression on the contractile apparatus. Subsequently, "fluorescence recovery after photobleaching" (FRAP) was applied to compare the dynamics of all protein variants in NRVC. After photobleaching, myopodin recovery time was in the range of $30 \mathrm{~s}$ in NRVC. Recovery times under unloaded conditions upon inhibition of contraction by treatment with the myosin inhibitor 2,3-butanedione monoxime (BDM) were significantly shorter. In contrast, increased contractility upon treatment with caffeine, significantly elevated recovery times.

Our results showed that all myopodin isoforms exhibited unexpectedly high mobile protein fractions and turnover rates. Inhibition, but not stimulation of contraction, induced alterations in myofibrillar morphology that were accompanied by an isoform-specific reduced mobility and increased translocation of myopodin to the nucleus. These data propose correlation of mechanical activity with the dynamic behavior of certain myofibrillar proteins.

Reza Mazar is a candidate for a young investigator award to be judged during the EMC 2012.

\section{0}

Myosin content in single muscle fibers from young and old men following disuse and recovery

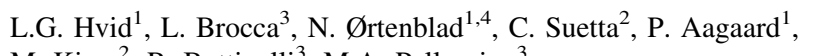
M. Kjaer $^{2}$, R. Bottinelli ${ }^{3}$, M.A. Pellegrino ${ }^{3}$

${ }^{1}$ University of Southern Denmark (SDU), SDU Muscle Research Cluster (SMRC), Institute of Sports Science and Clinical Biomechanics, Denmark; ${ }^{2}$ University of Copenhagen, Bispebjerg 
Hospital, Institute of Sports Medicine \& Center of Healthy Aging, Denmark; ${ }^{3}$ University of Pavia, Department of Molecular Medicine, Italy; ${ }^{4}$ Mid Sweden University, Swedish Winter Sports Research Centre, Department of Health Sciences, Sweden

Biopsies were obtained from vastus lateralis in 6 young ( 24 years) and 6 old (68 years) healthy men before and after 14 days of lower limb disuse (whole-leg casting), and after 28 days of active recovery (resistance training). Myosin heavy chain (MHC) isoform composition and quantification of myosin content $(n=349$ fibers) were determined by gel electrophoresis. In addition, single fiber specific force $\left(\mathrm{SF}=\mathrm{Ca}^{2+}\right.$-activated force per cross-sectional area) was measured before and after disuse ( $n=281$ fibers). Before disuse, MHC I and IIa single fiber myosin content were similar in young and old. After disuse, myosin content decreased in both MHC I (young: $-29 \%$ from $221 \pm 11 \mu \mathrm{M}$; old: $-19 \%$ from $243 \pm 10 \mu \mathrm{M})$ and IIa (young: $-23 \%$ from $204 \pm 12 \mu \mathrm{M}$; old: $-32 \%$ from $211 \pm$ $21 \mu \mathrm{M})$. Correspondingly, SF decreased in both MHC I (young: $-18 \%$ from $75.3 \pm 4.0 \mathrm{kN} / \mathrm{m}^{2}$; old: $-8 \%$ from $77.0 \pm 3.1 \mathrm{kN} / \mathrm{m}^{2}$ ) and IIa (young: $-22 \%$ from $117.3 \pm 6.1 \mathrm{kN} / \mathrm{m}^{2}$, old: $-20 \%$ from $117.4 \pm 7.9 \mathrm{kN} / \mathrm{m}^{2}$ ). After recovery, myosin content returned to baseline levels in MHC I (young: $255 \pm 14 \mu \mathrm{M}$; old: $244 \pm 14 \mu \mathrm{M}$ ). Similar observations occurred for MHC IIa in old $(225 \pm 20 \mu \mathrm{M})$, whereas a higher level was reached in young $(269 \pm 22 \mu \mathrm{M})$. The observed changes in the old were larger for MHC IIa versus I fibers.

In conclusion, 14 days of disuse decreased single fiber myosin content in both young and old (in particular for MHC IIa fibers), which in part explained the observed decreases in SF. Twenty-eight days of active recovery fully re-established myosin content in both young and old.

\section{1}

Effects of anabolic steroid on nuclear number and muscle mass

I.M. Egner ${ }^{1}$, J.C. Bruusgaard ${ }^{1,2}$, E.E.H. Hansen ${ }^{1}$, K. Gundersen ${ }^{1}$

${ }^{1}$ University of Oslo, Department of Molecular Biosciences, Oslo, Norway; ${ }^{2}$ Atlantis Medical College, Oslo, Norway

Anabolic steroids (AS) is a potent drug that increase muscle mass and it is widely misused by athletes because of its performance enhancing effects. In previous studies we have demonstrated that nuclei number after overload does not decrease after periods of inactivity. The aim of this study was to investigate if AS treatment lead to increase in number of nuclei and if previous use of anabolic steroids promotes muscle growth at a later time point.

Pellets containing AS or placebo were implanted subcutaneously in female NMRI mice. Mice were treated for 14 days before pellets were removed. After subsequently 21 days $2 / 3$ of m.tibialis anterior were removed to induce overload on m.EDL for 14 days. For measurements of cross sectional area (CSA) and counting of myonuclei, cryosections were stained with anti-dystrophin and DAPI. Analyzes were also preformed by in vivo intracellular injections.

Treatment with AS for 14 days leads to an increase in the CSA and the number of nuclei. The new nuclei seemed to be retained, even during subsequent removal of AS. The higher number of nuclei also seemed to facilitate muscle hypertrophy during overload.

\section{2}

Tropomyosin flexibility modulates $\mathrm{Ca}^{2+}$ sensitivity of thin filament and affects tension relaxation in skeletal muscle myofibrils after troponin-tropomyosin removal and reconstitution

B. Scellini ${ }^{1}$, C. Ferrara ${ }^{1}$, N. Piroddi ${ }^{1}$, J. Sumida ${ }^{2}$, C. Poggesi ${ }^{1}$, S.S. Lehrer ${ }^{2}$, C. Tesi ${ }^{1}$
${ }^{1}$ Università di Firenze, Dipartimento di Scienze Fisiologiche, Firenze, Italy; ${ }^{2}$ Boston Biomedical Research Institute, Watertown, MA, USA

Tropomyosin $(\mathrm{Tm})$ is a coiled-coil alpha-helix regulating the cooperative activation of muscle contraction by the thin filament. Tm forms strands along actin filaments which azimuthally move between switched-on/off locations under the influence of $\mathrm{Ca}^{2+}$-troponin $(\mathrm{Tn})$ and strong crossbridge formation. The flexibility of Tm strongly influences its movement so that the relaxation-activation mechanism within the thin filament may be critically modulated. We studied the mechanical consequences of the presence of chicken recombinant alpha tropomyosin, D137L, in rabbit skeletal muscle myofibrils, previously characterized in vitro. Endogenous Tm and Tn were replaced into rabbit skeletal muscle myofibrils with recombinant rabbit Tn (WT) and chicken alpha Tm (WT and L137D). Tm-Tn replacement was about $90 \%$ (SDS-PAGE). Force recordings from small bundles of myofibrils show that at saturating $\mathrm{Ca}^{2+}$ (pCa 4.5), maximal tension was not affected by Tm flexibility nor were the rates of force activation $\left(k_{\mathrm{ACT}}\right)$ and force redevelopment $\left(k_{\mathrm{TR}}\right)$. Interestingly, D137L Tm decelerates the rate of the fast phase of myofibril force relaxation. This is likely related to the higher " $\mathrm{Ca}^{2+}$-independent" tension observed in the D137L replaced myofibrils and then to the presence of some myosin heads able to cycle in the absence of $\mathrm{Ca}^{2+}$, resulting into slower relaxation and higher "resting" tension. Moreover, at submaximal $\mathrm{Ca}^{2+}$ (pCa 5.9), the presence of D137L Tm significantly increases (about 15-20\%) both force and kinetics of force generation. Consistently, force-pCa curves obtained from myofibrils replaced with $\mathrm{D} 137 \mathrm{~L}$ Tm showed a 0.15 increase in $\mathrm{pCa}$ compared to WT and an increased apparent cooperativity.

These results suggest that the presence in the sarcomere of D137L Tm could increase fractional occupancy of the thin filament Open State without $\mathrm{Ca}^{2+}$ (stronger affinity for $\mathrm{Ca}^{2+}$ than to the Blocked state) together with an increase in the cooperative unit size expected from a decrease in $\mathrm{Tm}$ flexibility. This result supports the hypothesis that the increased flexibility imparted to the Tm coiled-coiled structure by Asp at 137 avoids excessive turning-on of the system at the high physiological myosin concentrations.

Supported by STREP Project "BIG-HEART", 241577 EEC and NIH HL22461.

\section{3}

Increased intramuscular connective tissue in sarcopenia: influence on muscle specific force

J.S. McPhee ${ }^{1}$, T.M. Maden-Wilkinson ${ }^{1}$, G. De Vito ${ }^{2}$, H. Degens ${ }^{1}$, D.A. Jones ${ }^{1}$, M.V. Narici ${ }^{3}$

Manchester Metropolitan University, Institute for Biomedical Research into Human Movement and Health, Manchester, UK; ${ }^{2}$ University College Dublin, Institute for Sport and Health, Dublin, RoI; ${ }^{3}$ University of Nottingham, Faculty of Medical and Health Science, Derby, UK

The causes of reduced in vivo specific force (SF) in skeletal muscle weakness in old age has not been well characterised in humans. In young $(22 \pm 1$ years $)$ and older $(72 \pm 1$ years $)$ men $(n=36)$ and women $(n=35)$ we measured quadriceps in vivo SF as: (maximal torque/patellar tendon moment arm)/quadriceps physiological cross sectional area (PCSA) * the cosine of the fibre fascicle pennation angle. Data are reported as mean \pm SEM.

Compared with young, the older subjects were $37 \%$ weaker ( $246.5 \pm 11.7$ vs $156.2 \pm 7.1 \mathrm{Nm}$ in young and old, respectively); PCSA was $27 \%$ lower $\left(204.5 \pm 7.3\right.$ vs $149.6 \pm 5.9 \mathrm{~cm}^{2}$ in young and old, respectively), and SF was $17 \%$ lower (Fig. 1 ; all $P<0.0005$ ). The relative reduction in SF was similar in men and women. Muscle biopsy samples showed similar fibre-type composition and type I fibre 


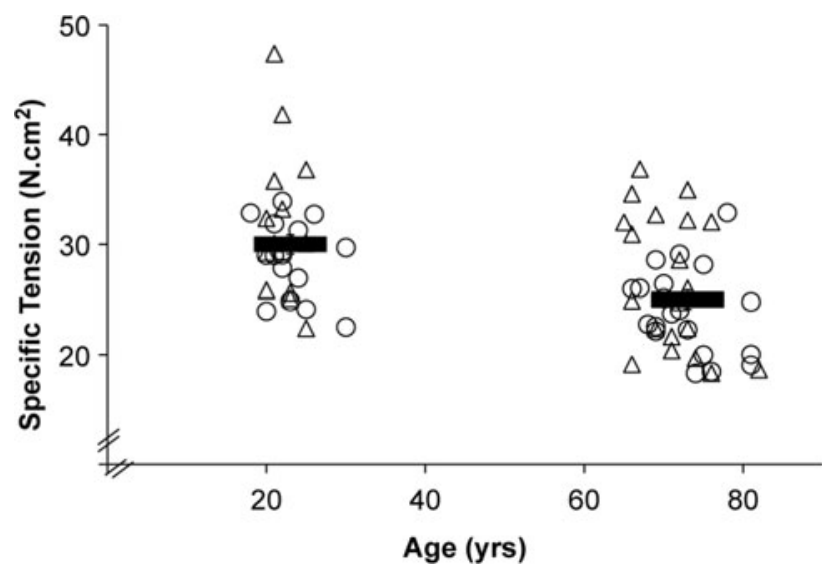

Fig. 1 Quadriceps in vivo specific force. Individual data points are shown for men (circles) and women (triangles). The mean for young and older groups are shown as black bars

cross sectional area in young and old, but $26 \%$ smaller type II fibres in old $(P<0.0005)$. Magnetic resonance imaging pixel intensity and histological collagen stain both indicated around $12 \%$ higher levels of connective tissue in older muscle and this was significantly correlated with SF ( $r=0.376 ; P=0.002)$. Adjusting the PCSA measurements of older people to take account of the increased connective tissue restored SF to within $92 \%$ of that of young.

It is concluded that increased intramuscular connective tissue content accounts for about half of the deficit in quadriceps SF by reducing contractile muscle mass in older people.

Supported by the EU Framework 7 Project MYOAGE: Understanding and Combating age related muscle weakness Grant Agreement Number: 223576.

\section{5}

Study of thin filament complexes by solid state NMR spectroscopy

N. Pace, M. Pfuhl

King's College London, Cardiovascular/Randall Division, London, UK

Actin plays a key role in muscle contraction. Even if the knowledge of the molecular mechanism and regulation of events driven by actin or actin binding is in continuous development, obtaining the atomic structure of the actin filaments and their complexes is a substantial challenge. In fact, only low resolution data has been obtained by electron microscopy and X-ray diffraction because the filamentous state prevents the use of the techniques which can provide data at atomic resolution, such as solution NMR spectroscopy or X-ray crystallography. Indeed, the size of the actin filaments exceeds the limit of detection of NMR and moreover they cannot be crystallized. Even if the low resolution data are enough to obtain general models of actin filaments, they have been unable to produce details for the characterization of the interactions which allow the development of the filaments. However, nowadays solid state NMR spectroscopy has reached a stage where atomic resolution data can be collected for protein complexes. In this study, initially we want to evaluate the potential of this technique to the structural study of F-actin and its complexes.

As a test system we study the complex of the Villain Head Domain (VHD), the $8 \mathrm{kDa}$ domain of ABLIM2 involved in actin interaction, and actin filaments. VHD domains have been are extensively characterized by solution NMR spectroscopy and due to its stability, expression yield and size has been chosen as a model protein for F-actin complexes.

We will show first results of using the VHD/F-actin interaction as a model system for the exploration of this newly emerging methodology.

\section{6}

Novel method of obtaining myofibrils from cardiomyocyte cultures

M. Jeong ${ }^{1}$, C. Ferrara ${ }^{2}$, C. Ferrantini ${ }^{2}$, R. Coppini ${ }^{3}$, B. Scellini ${ }^{2}$, N. Piroddi ${ }^{2}$, C. Tesi ${ }^{2}$, E. Cerbai ${ }^{3}$, C. Poggesi ${ }^{2}$

${ }^{1}$ University of Colorado, School of Medicine, Department of Medicine, Cardiology Section, Aurora, USA; ${ }^{2}$ University of Florence, Department of Physiology, Florence, Italy; ${ }^{3}$ University of Florence, Department of Pharmacology, Florence, Italy

Myofibril based mechanical studies allow targeted evaluation of sarcomeric protein function. Here we describe a novel method of obtaining myofibrils from primary cardiomyocyte culture. Adult rat ventricular myocytes (ARVMs) were obtained by enzymatic digestion and maintained in serum free environment for $72 \mathrm{~h}$. ARVMs were homogenized in relaxing solution (pCa 9.0) with $20 \%$ sucrose and myofibril suspension was made. Myofibrils, mounted in a force recording apparatus, were $\mathrm{Ca}^{2+}$-activated and fully relaxed by fast solution switching at $15{ }^{\circ} \mathrm{C}$ and $2.1 \mu \mathrm{m}$ initial sarcomere length. Results from ARVMs myofibrils were compared to those of myofibrils obtained from skinned ventricular tissue $(0.05 \%$ Triton-X100). At maximal $\mathrm{Ca}^{2+}$-activation (pCa 4.5) myofibril mechanical parameters from ARVMs were $8 \mathrm{mN} / \mathrm{mm} 2$ (resting tension), $156.4 \mathrm{mN} /$ $\mathrm{mm}^{2}$ (maximal active tension, Po), $5.4 \mathrm{~s}^{-1}$ (rate of force activation, $k_{\mathrm{ACT}}$ ) $5.2 \mathrm{~s}^{-1}$ (rate of force redevelopment following releaserestretch, $k_{\mathrm{TR}}$ ), $41.6 \mathrm{~ms}$ (linear relaxation duration), and $13.6 \mathrm{~s}^{-1}$ (exponential relaxation rate). The parameters of the myofibrils isolated from the ventricular tissue were not significantly different. Degree of run-down (decrease in Po and $k_{\mathrm{ACT}}$ between subsequent activation-relaxation cycles) was evaluated. Between first and third activations, we noted the following $\%$ changes in myofibrils from ARVM cultures: $-23.6 \%(\mathrm{Po}),-29.6 \%\left(k_{\mathrm{ACT}}\right)$. The magnitude of run-down was not statistically different between myofibrils from ARVMs versus the traditional Triton skinned samples. Finally, $\mathrm{pCa}_{50}$ from Triton skinned, ARVM culture day 0, and ARVM culture day 3 myofibrils were 5.76, 5.74, and 5.71, respectively.

Myofibrils obtained from ARVMs is a viable method to study myofibril mechanics. This culture based approach to obtaining myofibrils will allow pharmacological and genetic manipulation of the cardiomyocytes to correlate biochemical and biophysical properties.

\section{9}

\section{Structural and functional characterization of Ms1}

\section{Zaleska ${ }^{1}$, C. Fogl ${ }^{2}$, F. Wardle ${ }^{1}$, M. Pfuhl ${ }^{1}$}

${ }^{1}$ King's College London, Cardiovascular/Randall Division, London, UK; ${ }^{2}$ University of Leicester, Department of Biochemistry, Leicester, UK

Myocyte stress 1 (Ms1) also known as STARS (striated muscle activator of Rho signaling) and ABRA (actin binding Rho activator) is a protein discovered during induced left ventricular hypertrophy in rat hearts. It is expressed at low level in heart and skeletal muscle, however upon stress stimuli (e.g. high blood pressure) its expression increases 3 times, suggesting a possible involvement in the development of cardiac hypertrophy. 
In cardiomyocytes Ms1 lies alongside the Z-line of the I band, where it partially overlaps with $\alpha$-actinin and further investigation confirmed actin-binding properties of Ms1. It is proposed that Ms1 is involved in SRF- dependent transcription of muscle specific genes through regulating actin dynamics, however its complete mode of action is not fully understood.

Recently, the domain architecture of Ms1 was characterized in our lab and it revealed the presence of three independent parts: an unfolded $\mathrm{N}$-terminal, an unfolded actin-binding domain 1 (ABD1) and a folded actin- binding domain 2 (ABD2). Furthermore, the NMR structure of ABD2 contains a winged helix-turn-helix domain, which is commonly present in transcription factors and acts as a recognition motif in interaction with DNA. All of these findings suggest an alternative way of action of Ms1, where it could possibly be involved in gene transcription regulation, either through direct binding of regulatory DNA sequence, or by being a part of a transcription machinery complex.

In this work we will characterize interaction properties of Ms1 using gel retardation assay, SELEX and NMR spectroscopy. Additionally, the cellular localization of Ms1 will be studied using overexpression and immunofluorescence.

122

The Gly126Arg mutation of $\alpha$-tropomyosin increases the population of the strong-binding sub-states during the ATPase cycle

N.A. Rysev ${ }^{1}$, I.A. Nevzorov ${ }^{2}$, C.S. Redwood ${ }^{3}$, D.I. Levitsky ${ }^{2}$, Y.S. Borovikov ${ }^{1}$

${ }^{1}$ Institute of Cytology, RAS, St. Petersburg, Russia; ${ }^{2}$ A.N. Bach Institute of Biochemistry, RAS, Russia; ${ }^{3}$ University of Oxford, UK

It was hypothesized early that the well conserved non-canonical residue Gly-126 causes local destabilization of Tm. To test this, we labeled Cys190 of wild-type and Gly126Arg mutant $\alpha$-tropomyosins with the fluorescent probe 5-IAF, and Cys707 of myosin subfragment1 and Cys 374 of actin with 1.5-IAEDANS and F-actin with FITCphalloidin. These proteins were incorporated into ghost muscle fibres and their conformational states were monitored during the ATPase cycle by measuring polarized fluorescence.

Wild-type $\alpha$-tropomyosin increases the amplitude of the SH1 helix, subdomain-1 and actin monomer movements during the ATPase cycle, indicating the enhancement of the efficiency of the work of cross-bridges. The Gly126Arg mutation shifted tropomyosin further towards the open position, showing an increase in the binding of strong cross-bridges to actin during the ATPase cycle.

We propose that the highly conserved Gly-126 destabilizes the middle part of Tm, resulting in the concerted conformational changes of actomyosin. It is possible that the movement of mutant TM strands further towards the center of the thin filament is a reason for the enhancement of the strong-binding states during the ATPase cycle.

Supported by the Russian Fund for Fundamental Research N. 11-04-00244 and the Program 7 of the Presidium of the Russian Academy of Sciences.

\section{3}

The role of the M1592V mutation in the NaV1.4 $\mathrm{Na}^{+}$channels in the mechanism of hyperkalemic periodic paralysis

J.M. Renaud ${ }^{1}$, B. Lucas ${ }^{1}$, S. Khogali ${ }^{1}$, T. Ammar ${ }^{1}$, M. Barbalinardo ${ }^{1}$, L. Hayward ${ }^{2}$

${ }^{1}$ University of Ottawa, Department of Cellular and Molecular Medicine, Ottawa, Canada; ${ }^{2}$ University of Massachusetts Medical School, Worcester, USA
Hyperkalemic periodic paralysis (HyperKPP) is a disease of altered skeletal muscle excitability due to defective $\mathrm{Na}^{+}$channels (NaV1.4). It is characterized by myotonic discharges between and during periods of paralysis associated with elevated plasma $\left[\mathrm{K}^{+}\right]$. However, symptoms start in childhood as young as 1 year old and as late as 10 year old, followed by a worsening of the symptoms until adolescence. Hyper$\mathrm{KPP}$ is also associated with several changes in protein expression. So, the objective of this study was to determine if the late onset of the disease is due to when the cell membrane density of NaV1.4 channels reaches a critical level or if some symptoms are worsened by changes in the content/activity of other membrane components.

The NaV1.4 channel protein content measured by Western Blots reached a maximum by $3 \mathrm{wk}$. of age in both wild type and HyperKPP mice. However, by two weeks of age, HyperKPP EDL had a tetrodotoxin-sensitive $\mathrm{Na}^{+}$influx that was 3.6-times greater than wild type EDL. Furthermore, at the same time HyperKPP EDL had already defective contractile properties: lower tetanic force at normal and high $\left[\mathrm{K}^{+}\right]$e than wild type EDL. Neither the defective $\mathrm{Na}^{+}$influx nor contractility worsened between the ages of 0.5 to 12 months. Finally, the shift in fiber type toward type IIA occurred only by 2 mo. of age.

It is suggested that the HyperKPP symptoms are primarily related to the defective NaV1.4 channels and are not worsened by other changes in membrane components.

\section{4}

Hyperkalemic periodic paralysis symptoms vary between muscles and do not correlate with the content of NaV1.4 channels or $\mathrm{Na}^{+}$ influx

T. Ammar ${ }^{1}$, B. Lucas ${ }^{1}$, S. Khogali ${ }^{1}$, D. Dejong ${ }^{1}$, M. Barbalinardo ${ }^{1}$, J.M. Renaud ${ }^{1}$, L. Hayward ${ }^{2}$

${ }^{1}$ University of Ottawa, Department of Cellular and Molecular Medicine, Ottawa, Canada; ${ }^{2}$ University of Massachusetts Medical School, Worcester, USA

Hyperkalemic periodic paralysis (HyperKPP) is a disease of altered skeletal muscle excitability due to defective $\mathrm{Na}^{+}$channels (NaV1.4). It is characterized by myotonic discharges between and during periods of paralysis associated with elevated plasma $\left[\mathrm{K}^{+}\right]$. Interestingly, HyperKPP patients rarely suffer from respiratory distress despite the fact that the diaphragm expresses NaV1.4 channels. The objective of this study was therefore to compare how symptoms differ among muscles in an attempt to better understand how diaphragm can be asymptomatic. HyperKPP EDL had the highest NaV1.4 channel protein content being slightly lower in diaphragm (73\%), and much less in soleus (32\%) and FDB $(23 \%)$; i.e., in the order of EDL $>$ diaphragm $>$ soleus $\sim$ FDB. Surprisingly, the tetrodotoxin-sensitive $\mathrm{Na}^{+}$influx (i.e., through NaV1.4 channels) did not match the difference in nav14 channel content. It was the highest in diaphragm, slightly less in EDL and soleus and very low in FDB; i.e., in the order of diaphragm $>$ EDL $\sim$ soleus $\gg$ FDB. The contractile defect and changes in fiber type were the highest in EDL, lower in soleus and inexistent in diaphragm and FDB.; i.e., the severity of the contractile defect in HyperKPP skeletal muscles appeared to be in the order of EDL $>$ soleus $\gg$ diaphragm $\sim$ FDB.

The severity of HyperKPP symptoms matched neither the density of NaV1.4 channels in the cell membrane nor the $\mathrm{Na}^{+}$influx. Perhaps, other cell membrane components, especially in the diaphragm, somehow protect that muscle against HyperKPP symptoms.

\section{5}

Structural investigation of titin- $\alpha$-actinin interactions

V. Deineko ${ }^{1}$, L. Fiaschi ${ }^{2}$, E. De Almeida Ribeiro ${ }^{3}$, J. Trinick ${ }^{2}$, K. Djinovic-Carugo ${ }^{3}$, M. Wilmanns ${ }^{1}$ 
${ }^{1}$ European Molecular Biology Laboratory, Notkestraße 85, Build. 25A, 22603 Hamburg, Germany; ${ }^{2}$ University of Leeds, Institute of Molecular and Cellular Biology, Leeds LS2 9JT, UK; ${ }^{3}$ University of Vienna, Max F. Perutz Laboratories, Department for Biomolecular Structural Chemistry, Dr. Bohr-Gasse 9 (VBC 5), 1030 Vienna, Austria

The Z-disk is observed at electron microphotographs as a dense line forming the sarcomere boundaries in myofibrils of striated muscles. It performs the function of passive force transmission and serves as a nodal point of signaling within sarcomere. The key components of $Z$-disk macromolecular scaffold are actin, $\alpha$-actinin and titin. The Z-disk portion of titin contains short, highly similar repeats (Z-repeats or $\mathrm{Zr}$.). The number and type of these repeats vary due to differential splicing. Here, we are addressing the fundamental question of how the interactions of titin and $\alpha$-actinin form a distinct Z-disk pattern, both at the level of specific atomic interactions and at the level of the overall ultrastructural organization within the Z-disk. Our work builds on an initial investigation that was focused on the interaction of repeats 1 and 7 with the $\alpha$-actinin EF hand domains $1-4$ [1,2], which, however, was carried out in the absence of the physiological context of the sarcomeric Z-disk.

To date, we have purified various titin constructs, ranging from those with a single repeat to the longest with a total of six Z-repeats and complementary a-actinin constructs of different length. Our data, obtained so far, confirm established stoichiometries and $\alpha$-actinin preferences amongst the different titin $\mathrm{Zr}$ repeats. Further data of biophysical characterization and electron microscopy will be presented at the meeting. We will also discuss the evolutionary conservation of $\mathrm{Zr}$ repeats in titin of different vertebrates, providing further clues on the importance and level of conservation of these protein/protein networks in sarcomeres.

\section{References}

[1] Atkinson R., Joseph C., Kelly G., Muskett F., Frenkiel T., Nietlispach D., Pastore A. (2001) $\mathrm{Ca}^{2+}$-independent binding of an EF-hand domain to a novel motif in the $\alpha$-actinin-titin complex. Nature Structural Biology,Vol. 8, no. 10.

[2] Joseph C, Stier G, O'Brien R, Politou AS, Atkinson RA, Bianco A, Ladbury JE, Martin SR, Pastore A. (2001) A structural characterization of the interactions between titin Z-repeats and the alphaactinin C-terminal domain. Biochemistry. 40(16):4957-65.

Sponsored by MUSIC.

\section{6}

Structural insights into the dimeric assembly and interaction of myotilin in muscle Z-disc

A. Onipe ${ }^{1}$, N. Pinotsis ${ }^{1}$, I. Grishkovskaya ${ }^{1}$, D. Fürst ${ }^{2}$, K. Djinović-Carugo ${ }^{1}$

${ }^{1}$ University of Vienna, Department of Structural and Computational Biology, Max F. Perutz Laboratories, A-1030 Vienna, Austria; ${ }^{2}$ University of Bonn, Institute for Cell Biology, D-53121 Bonn, Germany

Myotilin is a $55 \mathrm{kDa}$ protein comprising of a 250 amino acid N-terminal domain followed by two immunoglobulin-like (Ig) domains. It is localized in the Z-disc of muscle sarcomere and has been associated with several myofibrillar myopathies. Even though it has been reported to interact with actin, $\alpha$-actinin-2, filamin C, ZASP, and FATZ/myozenin/ calsarcin $^{1,2}$, the structural integration of myotilin permitting multiple interactions still remains unclear. To gain insight into the overall architecture of myotilin and to understand the structural basis of its interactions, structural investigation was undertaken. Small angle X-ray scattering (SAXS) was employed for structural studies and chemical cross-linking/mass spectrometry for mapping the dimer interface residues. SAXS revealed an extended dimeric conformation and chemical cross-linking suggested that only Ig-2 is responsible for the dimerization. Further SAXS analysis provided an antiparallel model.

Our results suggest that myotilin adopts an extended, antiparallel dimeric conformation; an architecture which plausibly permits myotilin $\mathrm{N}$-termini to be anchored to $\alpha$-actinin-2, while the C-termini interact with ZASP PDZ domain, in a manner essential for signalling in the Z-disc.

\section{References}

[1] Salmikangas, P., et al. (2003). Hum Mol Genet 12(2).

[2] von Nandelstadh, P., et al. (2009). Mol Cell Biol 29(3).

Sponsored by: the MUZIC research network and FWF Austrian Science Funds-Doctoral Program W1221 Structure and Interaction of Biological Macromolecules.

\section{8}

Muscle to bone ratio reveals the extent of muscle atrophy and sarcopenia during ageing

J.S. McPhee ${ }^{1}$, T.M. Maden-Wilkinson ${ }^{1}$, D.A. Jones ${ }^{1}$, M.V. Narici ${ }^{2}$, H. Degens ${ }^{1}$

${ }^{1}$ Institute for Biomedical Research into Human Movement and Health, Manchester Metropolitan University, Manchester, UK;

${ }^{2}$ Faculty of Medical and Health Science, University of Nottingham, Derby, UK

In studies of sarcopenia the appendicular muscle mass is often normalised to height $\left(\mathrm{ALM} / \mathrm{h}^{2}\right)$ to compare young with elderly. However, height is reduced with ageing, so normalising muscle mass to height could underestimate muscle lost with ageing. We have validated the use of the femur cross-sectional-area (Fcsa) to normalise quadriceps muscle size and reveal the extent of quadriceps muscle lost with ageing.

In healthy young $(n=39$, mean age $22 \pm 3)$ and elderly men and women $(n=47$, mean age $72 \pm 5)$ DEXA was used to calculate $\mathrm{ALM} / \mathrm{h}^{2}$ and magnetic resonance imaging (MRI) used to measure quadriceps and femur size.

ALM $/ \mathrm{h}^{2}$ revealed that elderly muscle mass was $93 \%$ of young and only $2 \%$ of the elderly population had muscles $>2$ standard deviations lower than the mean of the young. The Fcsa did not differ significantly between young and elderly at any point along the shaft $(P=0.58)$. Normalising quadriceps cross-sectional-area to Fesa at mid-femur showed elderly quadriceps size to be $71 \%$ of younger muscle (Young: $12.4 \pm 1.33$, Elderly: $8.8 \pm 1.45 ; \quad P<0.001$ ). Moreover, $83 \%$ of the elderly had muscles $>2$ standard deviations smaller than the mean of young.

By expressing the muscle cross-sectional-area as a ratio of Fcsa we have shown the large majority $(83 \%)$ of healthy elderly to have quadriceps muscles that were substantially smaller than the muscles of young adults. Conventional DEXA identified only $2 \%$ of this population as having substantially smaller muscles than young.

Supported by the EU Framework 7 Project MYOAGE: Understanding and Combating age related muscle weakness Grant Agreement Number: 223576.

\section{3}

The evaluation of matrix -metalloproteinase -7 and -9 and their tissue inhibitors (TIMP-1 and TIMP-2) in patients with coronary syndromes with/without revascularisation interventions

F. Revnic ${ }^{1}$, C.R. Revnic ${ }^{2}$, A.S. Nica ${ }^{3}$, B. Paltineanu ${ }^{1}$, G. Prada ${ }^{2}$, G. Dinu ${ }^{4}$, C. Pena ${ }^{1}$, S. Prada ${ }^{1}$ 
${ }^{1}$ Biology of Aging, NIGG “Ana Aslan”, Bucharest, Romania;

${ }^{2}$ Ambroise Pare'Hospital, University of Medicine, Paris, France;

${ }^{3}$ UMF "Carol Davila", Bucharest, Romania; ${ }^{4}$ Floreasca Emergency Hospital, Bucharest Romania

Cardiac remodeling accounts for changes occuring at cellular and extracellular compartments, causing alterations of myocardium and chamber geometry. The aim of the study was to identify and characterize the enzymatic profile of matrix proteinases (MMP)-7 and -9 and their tissue inhibitors (TIMP-1 and TIMP-2), in patients with coronary artery disease (CAD), correlated with the patient's clinical status, cardiac function and revascularization interventions.

Total plasma MMP7, active fraction of MMP9, TIMP1 and TIMP2 were determined by ELISA method in 68 patients with confirmed CAD (Group A, $56.6 \pm 9$, LVEF $56.4 \pm 11 \%$ ) and compared with a control group of 23 patients free of cardiac pathology with normal coronary arteries (Group B, $58.1 \pm 10$, LVEF $58.7 \pm 5 \%$ ). LVEF and wall motion index (WMI) were computed. Diastolic function parameters were evaluated: mitral E/A ratio, E/E'septal ratio. We calculated global longitudinal (L), circumferential (C) and radial (R) strain (S) and strain rate (SR) values as the average of segmental values, by $2 \mathrm{D}$ strain analysis.

There were no significant differences in plasma MMP9, MMP9/ TIMP1 and MMP/TIMP2 ratio between normal and SA group, but only between normal and ACS group ( $P=0.02$ for MMP9). In group A, only MMP7, TIMP1/MMP7 and TIMP2/MMP7 ratio correlated with markers of systolic function: LVEF, WMI and global LS. Only active form of MMP9 and its ratio with TIMP- differed significantly in ACS. Total MMP7 and its ratio with TIMP correlated with parameters of LV systolic function even in controls with normal LVEF.

\section{4}

\section{Role of Fragile $X$ proteins in the heart}

S.M. Novak ${ }^{1}$, C.C. Gregorio ${ }^{1}$, D.C. Zarnescu ${ }^{2}$

${ }^{1}$ University of Arizona, Cellular and Molecular Medicine and Sarver Heart Center, Tucson, AZ, USA; ${ }^{2}$ University of Arizona, Molecular and Cellular Biology, Tucson, AZ, USA

RNA regulation provides a critical mechanism for controlling gene expression in the heart during normal development and disease. The RNA-binding Fragile X (FraX) protein, FXR1, has recently been linked to proper muscle structure and cardiac hypertrophy in mouse and zebrafish.

To decipher the direct role and mechanisms of FraX protein in the heart in vivo, we turned to the genetic capabilities of the Drosophila model, which expresses only one, functionally conserved FraX protein (dFmr1). To gain further insights into the role of dFMR1, we sought to identify a functional heart defect in Drosophila using video microscopy and a structural defect using immunofluorescence confocal microscopy. Using these approaches, we discovered that loss of dFMR1 causes a statistically significant decrease in heart rate and perturbs sarcomere structure. Expression of human FXR1 partially rescues the functional heart defect loss of function mutants, supporting functional conservation between species. Additionally, using Western blot analysis, we have discovered that loss of dFmr1 alters protein levels of known FXR1 targets: the costameric protein talin and the intercalated disc protein $\mathrm{ZO}-1$.

Taken together, these data suggest that dFmr1 has an important role in proper heart function, possibly by the translational regulation of its mRNA targets that encode integral cytoskeleton and cytoskeleton-associated proteins.

\section{5}

F-actin interaction with the C-terminus of cardiac troponin-I W.W.A. Mitchell ${ }^{1}$, V.B. Patchell ${ }^{1}$, B.A. Levine ${ }^{1,2}$

${ }^{1}$ University of Birmingham, College of Medical and Dental Sciences, Edgbaston, Birmingham, UK; ${ }^{2}$ University of Birmingham, School of Biosciences, Edgbaston, Birmingham, UK

Cardiac contractility is regulated by the interplay between $\mathrm{Ca}^{2+}$ and phosphorylation mediated via the troponin complex located on the F-actin/tropomyosin assembly. Actomyosin crossbridge formation is promoted by the calcium- dependent movement of the actomyosin ATPase inhibitory region of the troponin-I subunit (cTnI) away from actin while the $\mathrm{C}$ terminal region of $\mathrm{cTnI}$ is believed to stabilise the calcium-activated conformational changes in troponin- $\mathrm{T}$ and tropomyosin. The mechanism by which the C-terminus of $\mathrm{cTnI}$ influences the calcium-dependent position of tropomyosin (Tm) is still unresolved.

We here report on the F-actin and F-actin/Tm binding properties of the C-terminus of human cTnI using defined peptides spanning overlapping regions of the sequence (residues 147-209). The extreme C-terminal region of hcTnI (residues 198-209) bound to F-actin at a location distinct from the sites of F-actin interaction with the hcTnI inhibitory region and hcTnI residues $147-180$ that include the sonamed second $\mathrm{F}$-actin/Tm binding site. The $\mathrm{C}$-terminal extremity can therefore be considered as anchoring $\mathrm{cTnI}$ to F-actin, binding that was observed to localise the preceding segment (residues $\sim 179-195$ ) to the surface of F-actin without involving their direct interaction. The overall affinity of hcTnI residues 179-209 for F-actin-Tm was greater than for F-actin alone.

These observations suggest that hcTnI residues $\sim 179-195$ may contribute to stabilisation of the F-actin-tropomyosin complex via their association with tropomyosin, and that this relatively exposed protrusion from the surface of F-actin separates distinct F-actin binding patches.

\section{6}

\section{Stretch activation kinetics in hybrid fibre types of human skeletal muscle}

D. Neunhäuserer, S. Galler

University of Salzburg, Department of Cell Biology, Salzburg, Austria

Human skeletal muscles consist of different fibre types: slow fibres (slow twitch or type I) containing the myosin heavy chain isoform (MHC)-I and fast fibres (fast twitch or type II) containing MHC-IIa (type IIA) or MHCIId (type IID). The following order of decreasing kinetics is known: type IID>type IIA $\gg$ type I. This order is especially based on the kinetics of stretch activation, which is the most discriminative property among fibre types.

In this study we tested if hybrid fibres containing two MHC isoforms provide a transition in kinetics between flanking pure fibre types containing only one MHC isoform. We investigated stretch activation kinetics of single skinned fibres of various human muscles. After theses measurements, fibres were analyzed by high resolution SDS-PAGE for detecting their content of MHC isoforms.

Our data suggest that type $\mathrm{C}$ fibres, with different ratios of MHCIIa and MHC-I, do not provide a continuous transition between type IIA and type I fibres. Instead, the predominating isoform seems to determine the kinetics. In contrast, hybrid fibres, containing MHC-IId and MHC-IIa at different ratios, provide a somewhat continuous transition between type IID and type IIA fibres. However, also in 
these hybrid fibres, the predominating MHC isoform has a disproportionately high influence on stretch activation kinetics.

\section{7}

\section{Thin filament mutations in hypertrophic cardiomyopathy:} the role of allostery in a complex disorder

\section{J.C. Tardiff}

University of Arizona, Departments of Medicine and Cellular and Molecular Medicine, Tucson, Arizona, USA

Mutations in thin filament components of the cardiac sarcomere account for $\sim 20 \%$ of HCM. In order to develop more robust genotype-phenotype linkages, it is crucial to examine the most proximal effects of independent thin filament mutations at both the molecular and whole heart levels. In the current study we utilized well-characterized transgenic mouse models to elucidate the mechanism whereby mutations in cTnT result in blunting of the betaadrenergic response in vivo, a common clinical observation.

We utilized methods that addressed this complex physiologic response at multiple levels of experimental resolution including whole-heart physiology, $\mathrm{Ca}^{2+}$ kinetics performed on isolated adult ventricular myocytes and molecular dynamics simulations using our recently described all-atom model of the cardiac thin filament. Western analysis performed on the cTnT transgenics before and after isoproterenol infusion revealed that the incorporation of only the cTnT Arg92Leu mutation (not Del160Glu) in the thin filament altered the phosphorylation potential of cTnI at Ser 22/23. We hypothesized that the effect of this mutation (at a considerable physical distance from cTnI Ser 22/23) was mediated by allosteric changes in thin filament dynamics. Double-transgenics revealed the phosphomimetic cTnI only rescued relaxation in the Arg92Glu mice. Finally, MD simulations showed the orientation of cTnI and cTnC was altered such that accessibility of Ser22/23 was decreased in cTnT Arg92Glu complexes.

The blunted beta-adrenergic response in a subset of cTnT mutations is caused by a precise and allosterically mediated change in thin filament dynamics that alters PKA-mediated phosphorylation potential, a novel disease mechanism.

\section{8}

Phosphorylation of tropomodulin1 contributes to the regulation of actin filament architecture in cardiac muscle

K.T. Bliss ${ }^{1}$, T. Tsukada ${ }^{1}$, M.V. Dorovkov ${ }^{2}$, L. Kotlyanskaya ${ }^{3}$, S. Shah ${ }^{3}$, C.U. Nworu ${ }^{1}$, A.S. Kostyukova ${ }^{3}$, C.C. Gregorio ${ }^{1}$

${ }^{1}$ University of Arizona, Department of Cellular and Molecular Medicine, Tucson AZ, USA; ${ }^{2}$ Robert Wood Johnson Medical School, Department of Pharmacology, Piscataway NJ, USA; ${ }^{3}$ Washington State University, Department of Chemical Engineering and Bioengineering, Pullman WA, USA

The proper regulation of actin filament organization and cytoskeletal dynamics is extremely important for muscle function. Tropomodulin 1 (Tmod1) is an actin filament capping protein that is involved in pointed end thin filament regulation in striated muscle. Recently, Ser2 and Thr54 within Tmod1 were identified as phosphorylation targets by the kinase TRPM7 (transient receptor potential cation channel, subfamily M7) in vitro.

In this study, we identified a third phosphorylation site, Ser163, for the TRPM7 kinase in vitro. The significance of these newly discovered phosphorylation sites was assessed in rat cardiomyocytes using phosphomimic and phospho-deficient Tmod1 mutants. Triple phospho-mimic proteins [S2D T54E S163D] as well as triple phospho-off proteins [S2A T54A S163A] displayed faint and inconsistent pointed end assembly.
These proteins were also unable to restrict (reduce) thin filament lengths in the manner that wild type Tmodl does. However, these mutations do not alter the tropomyosin binding properties of Tmod1. Single mutant constructs either phospho-on or phospho-off ([S2A or $\mathrm{S} 2 \mathrm{D}]$ or [T54E or T54A] or [S163D or S163A]) displayed intermediate phenotypes. These mutants assembled more consistently and restricted thin filament lengths more efficiently than the triple phospho-on or phospho-off mutants, but not to the same degree as wild type Tmod1. FRAP analysis revealed that Tmod phospho-off [S2A T54A S163A] displayed altered association with thin filament pointed end.

Taken together, our data suggest that pointed end assembly of Tmod1 depends on the phosphorylation states of Tmod1 protein and that one of the mechanisms for thin filament length regulation can be linked to the dynamic phosphorylation of Tmod1.

\section{2}

Structure-function relationship of $\mathrm{Na}^{+}$-dependent activity of muscle-specific calpain

Y. Ono, E. Takaya, N. Doi, F. Kitamura, H Sorimachi.

Tokyo Metropolitan Institute of Medical Science, Department of Advanced Science for Biomolecules, Calpain project Tokyo, Japan

Calpain is an intracellular $\mathrm{Ca}^{2+}$-requiring cysteine protease and the human genome contains 15 different calpain genes. Among them, CAPN3 (previously called p94 or calpain-3) is specifically expressed in skeletal muscle. Since genetic defects in CAPN3 activity causes limb-girdle muscular dystrophy type 2A (LGMD2A), physiological importance of CAPN3 has been well accepted. Several different LGMD2A mouse models, i.e., knock-out $\left(\mathrm{Capn}^{-1-}\right)$ and knock-in $\left(\mathrm{Capn} 3^{\mathrm{CS} / \mathrm{CS}}\right)$ mice have been generated and shown to be valuable for studying mechanisms underlying LGMD2A pathology. On the other hand, CAPN3 has been a difficult subject for an enzymatic characterization due to its propensity to spontaneously autolyze when expressed in cultured cells other than skeletal muscle cells. Therefore, identification of factors other than $\mathrm{Ca}^{2+}$ which trigger the autolytic activation of CAPN3 and machineries specific to skeletal muscle cells attenuating autolysis of CAPN3 has been aimed.

One of the missense mutants of CAPN3 with attenuated autolytic activity, CAPN3:N358D, was expressed by the baculovirus system, purified, and used for enzyme characterization studies. For the identification of potenial CAPN3 substrates, quantitatve proteomics was performed using skeletal muscle tissues from wild type and knock-in mice. As a novel activating factor for CAPN3, we have found that $\mathrm{Na}^{+}$causes autolysis of CAPN3 in the absence of $\mathrm{Ca}^{2+}$. Furthermore, it was shown that complementarily activated CAPN3 at physiological concentrations. Mutagenesis studies showed that a conserved $\mathrm{Ca}^{2+}$ binding sequence in the protease acted as a $\mathrm{Na}^{+}$sensor and that much of the CAPN 3 molecule contributed to its $\mathrm{Na}^{+} / \mathrm{Ca}^{2+}$-dependent autolysis. Finally, proteomic analysis revealed that $\mathrm{Na}^{+}$and $\mathrm{Ca}^{2+}$ direct CAPN3 to proteolyze different substrates.

Since CAPN3 is the first example of intracellular $\mathrm{Na}^{+}$-activating protease, the in vivo mechanism how CAPN3 senses the increase of intracellular $\mathrm{Na}^{+}$is an important issue. One of the significance of the present results is that the imbalance in sensitivity of CAPN3 to $\mathrm{Na}^{+}$ and $\mathrm{Ca}^{2+}$ as well as the complete loss of its protease activity is potentially causative for LGMD2A.

\section{3}

The myomesin domain tandem My8-My9 confines helix-mediated elasticity to the C-terminal domain array My9-My13

S.D. Chatziefthimiou ${ }^{1}$, N. Pinotsis ${ }^{1}$, M. Rief ${ }^{2}$, G. Zoldak ${ }^{2}$, F. Beuron ${ }^{3}$, P. Konarev ${ }^{1}$, D. Svergun ${ }^{1}$, E. Morris ${ }^{3}$, M. Wilmanns ${ }^{1}$ 
${ }^{1}$ European Molecular Biology Laboratory Hamburg, Hamburg, Germany; ${ }^{2}$ Technical University of Munich, Institute for Biophysics and Munich Center for Integrated Protein Science, Germany; ${ }^{3}$ The Institute of Cancer Research, Chester Beatty Laboratories, Section of Structural Biology, London, UK

In striated vertebrate muscle, M-band ensures the packing regularity of the thick filaments and the uniform distribution of the tension across the myosin filaments during contraction. Myomesin isoforms, prominent components of the M-band, are filament connecting proteins considered to act as strain absorbers that keep thick filaments in register during muscle contraction. To unravel the mechanism of molecular elasticity in myomesin, we previously determined the overall architecture of its C-terminal part, including Ig domains My9, My10, My11, My12 and My13. Our data revealed a dimeric tail-totail filament structure that is folded into an irregular superhelical coil arrangement of almost identical domain modules, separated by exposed $\alpha$-helical linkers. Unfolding of these linkers, when tension is applied, can stretch this myomesin segment to about 2.5 times, returning to its original state when the tension is removed.

To gain further insight into the myomesin molecular architecture and identify the borders of its elasticity, we solved an additional crystal structure of the domains My8-My11. In contrast to the helical linkers connecting adjacent Ig domains in My9-My13, the Fn-III domain My8 is connected to the Ig domain My9 via a short unstructured linker, creating thus a tight interface between My8 and My9. This finding indicates that the established mechanism of myomesin filament elasticity is strictly confined to the C-terminal domain array My9-My13. The solution of the My8-My11 structure allowed us to generate an extended dimeric model for My8-My13, with an overall length of about $38 \mathrm{~nm}$, approaching thus further the M4-M4' distance of $44 \mathrm{~nm}$. Our new data present an important step to dissect different functions of myomesin along its highly repetitive domain structure.

\section{4}

A role for Grb10 as a regulator of muscle growth and glucose metabolism

N. Mokbel ${ }^{1}$, N. Turner ${ }^{1}$, R. Daly ${ }^{2}$, G.J. Cooney ${ }^{1}$, L.J. Holt ${ }^{1}$

${ }^{1}$ The Garvan Institute of Medical Research, Diabetes and Obesity Research Program; ${ }^{2}$ Cancer Research Program, Darlinghurst, Sydney, NSW, Australia

Grb10 is an intracellular adaptor protein which binds directly to several growth factor receptors and negatively regulates their action. Grb10-ablated mice exhibit enhanced glucose metabolism and increased muscle mass associated with an increase in myofibre number. To investigate the molecular mechanisms underlying the increase in muscle mass, we examined differences in proliferation of pure satellite cell populations isolated from wildtype (WT) and Grb10 knockout $(\mathrm{KO})$ muscle. We also investigated functional differences by examining insulin and IGF1 stimulated glucose uptake in differentiated myotubes. Mouse satellite cells purified with a CD56 marker were highly myogenic as shown by the expression of $\operatorname{pax} 7^{+} / \mathrm{MyoD}^{+}$ and muscle specific proteins after differentiation. We found no significant difference in mRNA expression of myogenic regulatory factors in Grb10 KO satellite cells compared to WT. Examination of the proliferation capacity of WT and KO satellite cells showed a twofold increase in mean population doubling of Grb10 KO cells compared to WT ( $n=4 \mathrm{WT}, 3 \mathrm{KO})$ after 3 days in culture. Glucose uptake was 1.8 fold higher $(P<0.05)$ in Grb10 KO primary myotubes compared to WT, independent of insulin or IGF1 action. The mRNA and protein expression of the main glucose transporters Glut 1 and Glut4 were unaltered in Grb10 KO primary myotubes.
These results support a role for Grb10 in regulating muscle glucose metabolism and suggest a novel role for Grb10 in influencing satellite cell proliferation which may have implications in treatment of muscle wasting conditions.

147

Comparison of power output of skinned skeletal muscle fibres from the cheetah and rabbit

C. Toepfer ${ }^{2}$, T. West ${ }^{1}$, R. Woledge ${ }^{1}$, N. Curtin ${ }^{2}$, P. Hudson ${ }^{1}$, J. Myatt ${ }^{1}$, M. Ferenczi ${ }^{2}$, A. Wilson ${ }^{1}$

${ }^{1}$ Royal Veterinary College London, Structure \& Motion Laboratory, North Mymms, UK; ${ }^{2}$ Imperial College London, National Heart and Lung Institute, Molecular Medicine Section, London, UK

We have compared the power output of muscle from cheetah and rabbit. We used muscle fibres skinned with glycerol and treated with Triton-X100. Single muscle fibres (1.5 mm long) were dissected from permeabilized muscle bundles and mounted between T-shaped clips. Sarcomere length was set to $2.4 \mu \mathrm{m}$. Fibre cross- sectional area was calculated from measurements of fibre width and thickness at 3 positions. Fibres were put into activating solution at $0{ }^{\circ} \mathrm{C}$ for $2 \mathrm{~s}$ followed by a rapid jump to $20{ }^{\circ} \mathrm{C}$ at which force develops. After isometric force had developed a step and ramp shortening was imposed at a range of velocities up to 7 fibre lengths $\mathrm{s}^{-1}$. The force and length changes of the contractile component were used to evaluate power. Fibres were kept for identification of fibre type. The force and power $v s$. velocity relationships were very similar for cheetah $(n=15,3$ outliers were rejected) and rabbit psoas $(n=7)$. The maximum power values were similar to those reported for intact, fast fibres from rat at $20{ }^{\circ} \mathrm{C}(1)$.

At $20{ }^{\circ} \mathrm{C}$ the maximum power that we measured was 4.3 times greater than that produced at $12{ }^{\circ} \mathrm{C}$ by skinned fibres from rabbit [2] and rat [3]. This reflects the strong temperature dependence of power output in the temperature range 10 to $20{ }^{\circ} \mathrm{C}$ reported by Ranatunga [1].

\section{References}

[1] Ranatunga, Experimental Physiology, 1998, 83, 371-376.

[2] He et al., Journal of Physiology, 1999, 517, 839-854.

[3] Bottinelli et al., Journal of Physiology, 1991, 437, 655-672.

\section{8}

Electron tomography of isolated honeybee Z-disks

\section{M.C. Rusu, J. Trinick}

University of Leeds, Institute of Molecular and Cellular Biology, Leeds, UK

Z-disks form the junctions between sarcomeres and transmit the force of contraction. In addition to its mechanical functions, the Z-disk has important roles in signaling pathways involving muscle growth and degradation. In order to understand all these roles in detail, the molecular organization of the Z-disk needs to be determined. Z-disks have been the object of several structural studies, usually visualized in plastic sections of muscle embedded in resin. However, the resolution achieved was $5-10 \mathrm{~nm}$, which is well below what is required to recognise protein shapes and also makes docking of component crystal structures difficult.

Isolated Z-disks were prepared by treating washed honeybee myofibrils with lactic acid or potassium iodide. Negative stain electron microscopy and electron tomography were carried out using FEI CM10 and Tecnai T12 microscopes. The preservation of the Z-disk lattice is assessed from the Fourier transforms of images, which 
showed clear reflections in a hexagonal pattern. Preliminary data indicate that isolated Z-disks are suitable specimens for cryo-electron tomography. Resolution achievable in raw tomograms is normally 40-50 A, but sub-tomogram averaging may improve this.

Sponsored by the participating research network MUZIC.

\section{0}

Structural details of muscle alpha-actinin complexed with its Z-disk binding partners

L. Fiaschi ${ }^{1}$, V. Deineko ${ }^{2}$, R.E. De Almeida ${ }^{3}$, M. Wilmanns ${ }^{2}$, K. Djinovic-Carugo ${ }^{3}$, J Trinick ${ }^{1}$

${ }^{1}$ University of Leeds, IMCB, Leeds, UK; ${ }^{2}$ European Molecular Biology Laboratory, Hamburg, Germany; ${ }^{3}$ University of Vienna, Department for Biomolecular Structural Chemistry, Max F. Perutz Laboratories, Vienna, Austria

The Z-disk is a specialized structure connecting adjacent sarcomeres in muscle cells. The Z-disc largely consists of $\alpha$-actinin providing a backbone for the insertions of actin based thin filaments as well as titin and other muscle proteins. $\alpha$-actinin is not simply a rigid spacer between actin filaments, but rather a flexible cross-linking, scaffolding, and anchoring protein that may contribute to tension sensing. The Z-disk has been the subject of many structural studies for many years, but the resolution of these has always been worse than $4 \mathrm{~nm}$ and so little is known about its detailed layout. A number of different models have been proposed but these do not deliver a satisfactory answer to the pressing problems regarding the intermolecular organization and interactions in the Z-disk. Flexible macromolecules, like alpha-actinin complexes, pose special difficulties for structure determination by crystallography or NMR. Progress can be made by electron microscopy and Single Particle reconstruction methodologies.

Here, we describe how we have used negative staining and singleparticle image processing techniques to explore the structure and flexibility of single molecules of $\alpha$-actinin complexed with Z-repeats of titin, attaining $\sim 2 \mathrm{~nm}$ resolution.

\section{1}

\section{Mechanics of sarcomeres and myofibrils isolated from striated muscles}

\section{D.E. Rassier}

McGill University, Departments of Kinesiology, Physiology and Physics, Montreal, Canada

Muscle contraction is driven by the interaction between myosin and actin filaments, and has characteristic features including a historydependence of force production after stretching or shortening, and the relations between force, sarcomere length and velocity of contraction. The mechanisms responsible for these features manifest differently according to the experimental preparation, which may lead to controversial interpretations.

We developed preparations to investigate the mechanics of (i) myofibrils containing a pre-determined number of sarcomeres inseries, (ii) individual sarcomeres isolated from myofibrils, and (iii) synthetic and natural myosin-actin filaments attached to microfabricated cantilevers. While these preparations provide data that are highly consistent and complementary, the results from mechanical experiments are instigating and do not always provide the same conclusions.

Our data confirm that the force produced during muscle stretching and shortening follows a force-velocity relation that is closely associated with the kinetics of myosin ADP release. However, it suggests that the long-lasting effect of stretching and shortening is associated with half-sarcomere length non-uniformities, and a sarcomeric component linked to a $\mathrm{Ca}^{2+}$-induced increase in the stiffness of titin molecules.

\section{3}

Using Xenopus to elucidate the roles of thin filament regulatory proteins: leiomodin $3(\operatorname{Lmod} 3)$ and tropomodulin $4(\operatorname{Tmod} 4)$ are critical for skeletal muscle thin filament assembly

\section{C.U. Nworu, D.C. Schnurr, C.C. Gregorio, P.A. Krieg}

The University of Arizona, Department of Cellular and Molecular Medicine and the Sarver Heart Molecular Cardiovascular Research Program, College of Medicine Tucson, Arizona 85724, USA

To generate optimal force for efficient contraction, actin-thin filament lengths must be closely regulated. Capping proteins contribute to thin filament length uniformity by regulating both elongation and depolymerization of the filaments at their ends. The Leiomodins (Lmod13) are structurally related to Tropomodulin (Tmod) proteins and are reported to localize to the pointed end of actin filaments and to contribute to thin filament length regulation. This study aims to determine the role of Tmod4 and Lmod3 during de novo skeletal myofibrillogenesis in Xenopus embryos. Developmental in situ hybridization studies show that only Lmod3 and Tmod4 transcripts are expressed at high levels in skeletal muscle from the earliest stages of development, suggesting that both molecules contribute to myofibril assembly. Reducing the protein levels of Tmod4 or Lmod3 via morpholino technology compromised sarcomere development, thin filament assembly and the embryo's ability to swim. Co-injecting either exogenous Lmod3 or Tmod4 mRNA together with their specific morpholino rescues the Lmod3 or Tmod4 morpholino induced perturbations, respectively. Myofibril structure is restored and embryos are able to regain $\geq 50 \%$ of their swimming ability.

Our results not only indicate that both $\operatorname{Lmod} 3$ and Tmod4 are critical for myofibrillogenesis in developing Хеnopus skeletal muscle, but also establish the Xenopus model system as a powerful platform to decipher mechanisms contributing to thin filament regulation.

\section{4}

From precursor muscle cells to fast fiber-type: a defined fiber-type specific mouse model

\section{Guerrero, J.J. Arredondo, M. Cervera}

Universidad Autónoma de Madrid. Instituto de investigaciones Biomédicas Alberto Sols C.S.I.C. Facultad de Medicina. Madrid, Spain

The absence of experimental and genetic tools to track precursor muscle cells, fiber-type conversions and muscle dynamics in vivo have impeded to better understand myogenesis in development, aging and in response to external or pathological stimuli. We have developed an excellent tool for rapid tracking of precursor muscle cells from somites to limbs or body wall and to examine directly primary and secondary myogenesis.

We have created transgenic mouse lines, carrying FURE/FIRE enhancer elements (Guerrero et al, 2010) from the mouse $f T n I$ gene upstream of the GFP reporter gene, to drive the expression of fluorescent markers to precursor muscle cells and fast-fibers. This will enable the use of non-invasive fluorescence imaging technology in live anesthetized embryos (Fig. 1) and animals to track precursor muscle cells and assess changes in fast-fibers within a muscle in the same animal in response to different stimuli. Here, we present fibertype characterization of the embryo and mouse transgenic lines. Our studies revealed that GFP expression correlates very well with the 


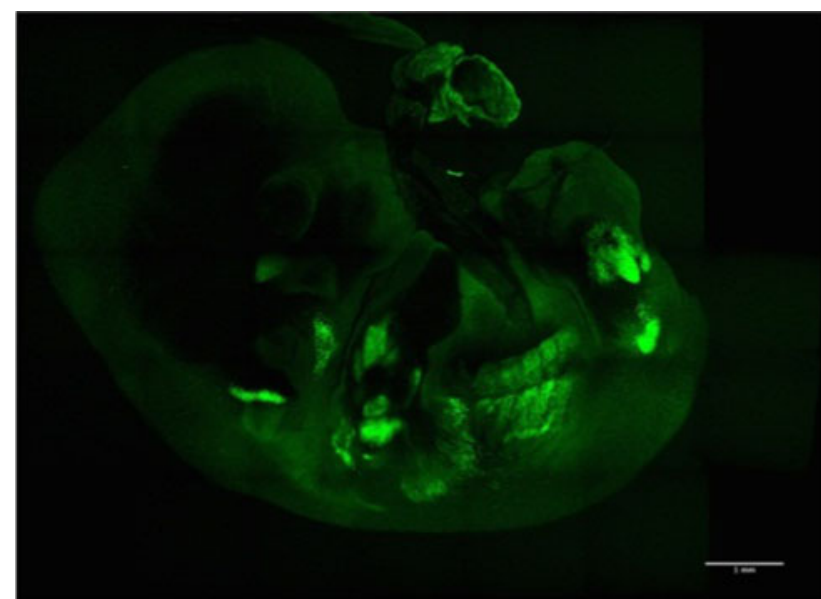

Fig. 1 GFP precursor muscle cells and primary myotubes are visualized directly migrating to the limbs and to the wall body in mouse whole embryo of $12.5 \mathrm{dpc}$

endogenous fTnI expression. GFP-expressing fibers are exclusively fast-twitch fibers. Type IIx fibers express higher GFP quantities. We also will present hindlimb muscles and diaphragm maturation in P1, P7 \& P14 mice. GFP gradation mirrors that of adulthood. Fast myosin appears while slow myosin expression decreased over time. This switch from slow MHC to fast MHC occurred earlier in diaphragm than in the rest of skeletal muscles. The FUFI-EGFP transgenic mice are an excellent tool for rapid tracking of precursor muscle cells and fast-twitch fibers.

\section{6}

\section{Structural insights into the conformational changes of human} Z-disk alpha-actinin-2

E.A. Ribeiro $\mathrm{Jr}^{1}$, K. Pirker $^{2}$, A. Salmazo ${ }^{1}$, N. Pinotsis ${ }^{1}$, C. Schreiner ${ }^{1}$, A. Ghisleni ${ }^{3}$, P. Konarev ${ }^{4}$, D.I. Svergun ${ }^{4}$; M. Gautel ${ }^{3}$,

K. Djinović-Carugo ${ }^{1}$

${ }^{1}$ University of Vienna, Department of Structural and Computational Biology, Max F. Perutz Laboratories, Campus Vienna Biocenter 5, A-1030 Vienna, Austria; ${ }^{2}$ Austrian Institute of Technology, Department of Health and Environment, Environmental Resources and Technologies, GmbH, A-3430 Tulln, Austria; ${ }^{3}$ King's College London, New Hunt's House Guy's Hospital, London SE1 1UL, UK; ${ }^{4}$ EMBL-Hamburg c/o DESY, Notkestrasse 85, D-22603, Hamburg, Germany

The Z-disk forms the lateral boundaries between adjacent sarcomeres in striated muscle. Apart from its major components alpha-actinin and F-actin, the Z-disk comprises a number of proteins and acts as a platform for a complex and dynamic web of interactions. Alpha-actinin cross-links anti-parallel actin filaments from adjacent sarcomeres. Muscle isoforms of alpha-actinin (ACTN2 and 3) are regulated by phosphoinositides, triggering a conformational change that renders alpha-actinin capable of binding to titin and actin (and some other Z-disk partners). This mechanism, essential for the organization of protein-protein network in the mature Z-disk, still remains elusive at molecular level. Here, the combination of structural and molecular biology, and biophysical techniques is being used to understand the molecular mechanism of how alpha-actinin-2 responds to phospholipids (e.g. phosphatidylinositol-4, 5- bisphosphate, $\mathrm{PIP}_{2}$ ). Fluorescence polarization experiments show that alpha-actinin- 2 and
PIP2 form a complex in vitro. The dissociation constant of this interaction is in the micromolar range, which is similar to the affinity values reported for other actin binding proteins and phospholipid complexes. Site-directed mutagenesis studies on alpha-actinin-2 are underway to map the key amino acid residues involved in this interaction. These results together with $\mathrm{X}$-ray crystallography, small angle scattering and electron paramagnetic resonance (EPR) spectroscopy experiments provide new insights into the molecular mechanism of conformational changes induced upon alpha-actinin-2 binding to $\mathrm{PIP}_{2}$ and other selected Z-disk proteins.

\section{8}

Structural characterization and interaction studies of myopodin isoform $\mathrm{A}$ with $\mathrm{Z}$-disc binding partners

E.H.S. Bezerra ${ }^{1}$, I. Grishkovskaya ${ }^{1}$, N. Pinotsis ${ }^{1}$, P.F. van der Ven ${ }^{2}$, D.O. Fürst ${ }^{2}$, K. Djinović-Carugo ${ }^{1}$

${ }^{1}$ University of Vienna, Department of Structural and Computational Biology, Max F. Perutz Laboratories, Vienna, Austria; ${ }^{2}$ University of Bonn, Department of Molecular Cell Biology, Bonn, Germany

Z-discs delineate the lateral borders of sarcomeres which are the smallest functional units in striated muscle cells. Myopodin is a 136 $\mathrm{KDa} Z$-disc protein that colocalizes with filamin and $\alpha$-actinin during all stages of muscle development. Although its interactions with several Z-disc proteins (Linnemann, et al. 2010). We set for a biochemical and structural characterization of myopodin and its interactions with Z-partners, employing an integrative approach encompassing different biophysical, biochemical, and structural methods such as isothermal titration calorimetry (ITC), differential scanning fluorimetry (DSF), X-ray crystallography and lower resolution structural techniques. PDZ domain and to central region constructs of myopodin have been successfully expressed and purified. Crystal structure of PDZ domain in complex with the C-terminal peptide of synemin has been solved at $2.0 \AA$ resolution. The structure of PDZ domain-synemin peptide complex revealed the classical binding in $\beta$-sheet augmentation mode. The peptide in the complex is bound in an anti-parallel fashion to the $\beta$-strand, with the C-terminal residue occupying a hydrophobic pocket on the PDZ domain classified as type 3. Bezerra is a candidate for a young investigator award to be judged during the EMC 2012.

\section{References}

Linnemann, A., P. F. van der Ven, et al. (2010). Eur J Cell Biol 89(9): 681-692.

\section{9}

Forces produced by activated muscle fibres during and after imposed length changes-effects of $\mathrm{Ca}^{2+}$ concentrations and MgADP

F.C. Minozzo ${ }^{1}$, D.E. Rassier ${ }^{1,2}$

${ }^{1}$ McGill University, Department of Kinesiology and Physical Education, Montreal, Canada; ${ }^{2}$ McGill University, Department of Physiology, Department of Physics, Meakins-Christie Laboratories, Montreal, Canada

We compared the effects of $\mathrm{Ca}^{2+}$ and MgADP activation on the force produced during and after length changes in skeletal muscles. Two sets of experiments were performed, where single fibres isolated from psoas were either (i) activated at $\mathrm{pCa}^{2+}$ of 4.5 and 6.0 , or (ii) 
activated at $\mathrm{pCa}^{2+} 4.5$ before and after administration of $10 \mathrm{mM}$ $\mathrm{MgADP}$. Fibres were activated isometrically at sarcomere lengths (SL) of 2.7, 2.8 and $3.0 \mu \mathrm{m}$. Fibres activated at 2.7 and $3.0 \mu \mathrm{m}$ were subsequently stretched or shortened ( $5 \% \mathrm{SL}$ at $1.0 \mathrm{SL} \mathrm{s}^{-1}$ ) to reach a SL of $2.8 \mu \mathrm{m}$. The kinetics of force during stretch was not altered by $\mathrm{Ca}^{2+}$ concentrations or MgADP, but the fast change in the slope of force development (P1) observed during shortening and the corresponding $\mathrm{SL}$ extension (L1) were higher at $\mathrm{pCa}^{2+} 6.0(\mathrm{P} 1=0.22 \pm 0.02 \mathrm{Po}$; $\left.\mathrm{L} 1=5.26 \pm 0.24 \mathrm{~nm} . \mathrm{HS}^{1}\right)^{1}$ than at $\mathrm{pCa}^{2+} 4.5(\mathrm{P} 1=0.15 \pm 0.01 \mathrm{Po}$; $\left.\mathrm{L} 1=4.48 \pm 0.25 \mathrm{~nm} \cdot \mathrm{HS}^{1}{ }^{1}\right) . \quad \mathrm{MgADP}$ increased L1 $\quad$ (5.52 \pm $\left.0.20 \mathrm{~nm} . \mathrm{HS}^{.1}\right)$, without altering P1. Forces after stretch and shortening were higher and lower, respectively, than the isometric forces at the corresponding SL. Force enhancement was lower at $\mathrm{pCa}^{2+} 4.5$ $(14.9 \pm 5.4 \%)$ than at $\mathrm{pCa}^{2+} 6.0(38.8 \pm 7.5 \%)$. Force depression after shortening was similar in both $\mathrm{Ca}^{2+}$ concentrations. Force enhancement and force depression were not affected by MgADP. These results suggest that $\mathrm{Ca}^{2+}$ concentration modulates the force enhancement independently from crossbridges, and that the number of attached crossbridges and the amount of strong-binding crossbridges influence force during shortening.

\section{0}

Mechanisms of myofibril and sarcomere formation in Drosophila adult muscles

\section{A. Kaya-Copur, F. Schnorrer}

Max Planck Institute of Biochemistry, Muscle Dynamics, 82152 Munich-Martinsried, Germany

Adult Drosophila possesses various different muscles such as cardiac muscles, striated body muscles, and specialized indirect flight muscles. Similarly to vertebrates, adult Drosophila muscles can be formed by multiple myofibers with many myofibrils. Interestingly, most structural components important for formation or function of vertebrate sarcomeres are conserved in Drosophila suggesting that the molecular mechanisms of their formation are similar.

Muscle movements are generated by their smallest building blocks, the sarcomeres. I aim to understand how myofibrils and sarcomeres of adult Drosophila are built. To identify key regulators, with bioinformatics approach I preselected genes containing cytoskeleton and sarcomere related domains, and combined these with systematic genome-wide RNAi data for muscle function [1]. This led to 195 candidates whose role in muscle formation is being tested with muscle-specific RNAi. To analyze sarcomere morphology, I image mature muscles with fluorescently tagged sarcomeric markers in vivo comparing fibrillar flight muscles to morphologically distinct tubular leg and abdominal muscles.

I identified genes specifically required for formation of either tubular or fibrillar muscles. Muscle-specific knockdown of an EF-hand domain containing uncharacterized gene results in dramatic reduction of abdominal and leg muscles with irregular sarcomeres, whereas flight muscles are not affected. However, another uncharacterized gene with predicted PDZ, SH3 and kinase domains affects only the flight muscles. The number of flight muscles is reduced; the myofibrils of the remaining fibres align with each other resembling the organization of tubular muscles.

Further characterization of these genes will help us to understand the mechanisms of sarcomere and myofibril formation.

\section{References}

[1] Schnorrer F, Schonbauer C, Langer CCH, Dietzl G, Novatchkova M, et al. (2010) Systematic genetic analysis of muscle morphogenesis and function in Drosophila. Nature 464, 287-291.

\section{2}

Cellular validation of the pseudoligand regulation model of the alpha-actinin titin interaction

A. Ghisleni ${ }^{1}$, R.E. de Almeida ${ }^{2}$, N. Pinotsis ${ }^{2}$, K. Djinović-Carugo ${ }^{2}$, M. Gautel ${ }^{1}$

${ }^{1}$ King's College London BHF of Research Excellence Centre, Randall Division for Cell and Molecular Biophysics and Cardiovascular Division, New Hunt's House, Guy's Campus, London SE1 1UL, United Kingdom; ${ }^{2}$ Max F. Perutz Laboratories-University of Vienna, Department of Structural and Computational Biology, Biocenter 5, A-1030 Vienna, Austria

$\alpha$-actinin 2 is a fundamental component of the striated muscle sarcomere. It localizes in the Z-disk and acts as an actin cross-linking protein, but also interacts with several partners. One of these interactions is with the repetitive motifs of the giant ruler protein titin, called Z-repeats, which appears to be the key interaction for the correct targeting of $\alpha$-actinin 2 to the Z-disk. Several lines of evidence show that the $\alpha$-actinin 2 homodimer is not able to bind titin Z-repeats, except in the presence of phosphatidylinositol-bisphosphate $\left(\mathrm{PIP}_{2}\right)$. In the model proposed by Young and Gautel (2001), the binding of $\mathrm{PIP}_{2}$ to the $\mathrm{N}$-terminal actin-binding domain induces the transition from a closed to open conformation, in which the C-terminal domain is free to interact with the Z-repeats. Based on the solved X-ray structure of the $\alpha$-actinin dimer, we designed mutations to disrupt the hydrophobic contacts that direct the EF-hands 3 and 4 to the linker region between the $\mathrm{ABD}$ and the rod region. This constitutively open $\alpha$-actinin2 variant is able to interact with titin-ZR7 in vitro, bypassing the $\mathrm{PIP}_{2}$ regulatory effect. The transfection of the same variant into neonatal rat cardiomyocytes induces a strong dominant-negative effect on the nascent Z-disk and the overall organization of the sarcomere is impaired, including the organization of actin.

These findings suggest a further regulatory role of PIP2, not limited only to the regulation of the $\alpha$-actinin $2 /$ titin interaction, and corroborate the pseudoligand regulation mechanism for $\alpha$-actinin 2 .

\section{References}

Young P, Gautel M (2000) The interaction of titin and alpha-actinin is controlled by a phospholipid-regulated intramolecular pseudoligand mechanism. Embo J. 19:6331-6340.

\section{4}

Plin2 expression in human skeletal muscle is associated with inactivity and aging

M. Conte ${ }^{1}$, E. Bellavista ${ }^{1}$, G. Trisolino ${ }^{2}$, E. Martucci ${ }^{2}$, E. Vasuri ${ }^{3}$, A. Degiovanni ${ }^{3}$, A. D’Errico-Grigioni ${ }^{3}$, R. Clima $^{1}$, A. Santoro ${ }^{1}$, A.B. Maier ${ }^{4}$, S. Salvioli ${ }^{1}$, C. Franceschi ${ }^{1}$

${ }^{1}$ University of Bologna, Dept. of Experimental Pathology and CIG-Interdepartmental Center "L. Galvani", Bologna, Italy; ${ }^{2}$ Reconstructive Hip and Knee Joint Surgery, Istituto Ortopedico Rizzoli, Bologna, Italy.; ${ }^{3}$ University of Bologna, Pathology Unit of the "F. Addarii" Institute of Oncology, S.Orsola-Malpighi Hospital, Bologna, Italy; ${ }^{4}$ Leiden University Medical Center, Dept. of Gerontology and Geriatrics, Leiden, Netherlands

Fat infiltration in skeletal muscle is associated with aging and physical inactivity. High levels of inter-muscular adipose tissue contribute to muscle quality decline, predict sarcopenia and increase risk of mobility impairment. Much less is known about lipid deposition within muscle fibres and muscle function. Intracellular lipid droplets are associated with a number of proteins, among which Perilipins (Plins) play a critical role in regulating intracellular lipid storage and 
mobilization. In particular, Plin2 is expressed in human skeletal muscle. This study aimed at investigating the different expression level of Plin2 in skeletal muscle from subjects of different age, either healthy or affected by mobility-limitating pathologies, such as osteoarthrosis or hip displasia. In the framework of the EU 7th Program Project MYOAGE, we collected and analysed biological samples from active and sedentary individuals and from hip-surgery patients of different age ( 20 to over 80 years).

Plin2 expression and distribution in muscle Vastus lateralis were investigated with Real-Time PCR, WB and IHC. We found that Plin2 expression is highly associated with low mobility independently from BMI. This phenomenon is even more evident in old people, where active individuals have low levels of Plin2 (only slightly higher than young people), while sedentary subjects or patients with low mobility display a dramatic increase of this protein. Furthermore, among inactive subjects, an inverse association is present between muscle strength and Plin2 expression.

These results suggest that a maintained physical activity can preserve skeletal muscle from intra-fibre lipid deposition even in old age.

Supported by the EU Framework 7 Project MYOAGE: Understanding and Combating age related muscle weakness Grant Agreement Number: 223576.

\section{5}

Leiomodin2 is an antagonist of tropomodulin1 at the pointed end of the thin filaments in cardiac myocytes

C.T. Pappas ${ }^{1}$, T. Tsukada ${ }^{1}$, N. Moroz ${ }^{2}$, P.B. Antin ${ }^{1}$, A.S. Kostyukova ${ }^{2}$, C.C. Gregorio ${ }^{1}$

${ }^{1}$ University of Arizona, Department of Cellular and Molecular Medicine, Sarver Heart Center, Tucson, AZ, USA; ${ }^{2}$ Washington State University, School of Chemical Engineering and Bioengineering, Pullman, WA, USA

Regulation of actin filament assembly is essential for efficient contractile activity in striated muscle. Leiomodin (Lmod) is an actinbinding protein and homolog of the actin filament pointed end capping protein, tropomodulin (Tmod). These proteins are structurally similar, sharing a common domain organization that includes two actin-binding sites. Lmod also contains a unique C-terminal extension that has a third actin-binding $\mathrm{WH} 2$ domain. The striated muscle specific isoform of $\operatorname{Lmod}(\operatorname{Lmod} 2)$ was reported to be an actin nucleator in cardiomyocytes.

Here we have identified a novel function of $\operatorname{Lmod} 2$ in the regulation of thin filament lengths. We show that Lmod2 localizes to the pointed ends of thin filaments where it competes for binding with Tmod1. Overexpression of Lmod2 results in loss of Tmod1 assembly and elongation of the thin filaments from their pointed ends. Interestingly, overexpression of Tmod1 has the opposite effect resulting in thin filament shortening (as published by others previously). We also discovered that Lmod2's WH2 domain is required for thin filament lengthening since its removal results in a molecule that caps the pointed ends similar to Tmod1. Furthermore, Lmod2 transcripts are first detected in the heart after it has begun to beat, suggesting that Lmod2's primary function is to maintain thin filament lengths in the mature heart.

Together, our data reveal that $\operatorname{Lmod} 2$ antagonizes the function of Tmod 1 and together these molecules appear to fine-tune thin filament lengths.

\section{6}

The role of tropomyosin heterodimers in the regulation of muscle contraction in cardiomyopathies

M. Janco, A. Kalyva, M.A. Geeves

School of Biosciences, University of Kent, UK
Hypertrophic cardiomyopathy (HCM) is the leading cause of sudden cardiac death in young adults. HCM can result from missense mutations in the gene encoding skeletal $\alpha$-tropomyosin (Tm). To understand how specific mutation leads to HCM, the recombinant $\alpha \mathrm{Tm}$ homodimers carrying HCM mutations have been intensively studied. However due to the heterozygous character of myocytes both wild type and mutant $\mathrm{Tm}$ are expressed in the affected individual. Therefore there is a potential formation of heterodimers.

We examined extent of in vitro heterodimers formation of a D175 N and E180G HCM mutations in only one chain of $\alpha \mathrm{Tm}$ dimer and compared heterodimer properties with WT and mutant homodimers.

Both D175N and E180G Tm HCM mutations do not show any preference in heterodimer formation with WT $\alpha \mathrm{Tm}$. The thermal stability of D175 $\mathrm{N}$ homo and heterodimer is indistinguishable from the WT homodimer. The affinities of D175 $\mathrm{N}$ heterodimer and WT for actin are identical whereas the D175 $\mathrm{N}$ homodimer shows 2 fold weaker affinity. Thermal stability of E180G Tm homodimer is significantly reduced while the heterodimer shows an intermediate reduction of stability in comparison to WT. Similarly the E180G homodimer shows 2 fold lower affinity for actin and the heterodimer affinity is intermediate. The in vitro kinetic assays show a $0.1-0.14$ pCa higher calcium sensitivity for the D175 $\mathrm{N}$ heterodimers and the D175 N and E180G homodimers than the WT. E180G Tm homodimer calcium sensitivity is the same as WT.

The results reveal distinct properties between homo and heterodimers. These differ depending on the mutation.

Janco is a candidate for a young investigator award to be judged during the EMC 2012.

169

Quantitative non linear optical microscopy to study the increase in fibrosis during cardiac disease progression

V. Caorsi ${ }^{1}$, C. Toepfer ${ }^{1}$, M. Sikkel ${ }^{2}$, A.R. Lyon $^{2}$, K. MacLeod ${ }^{2}$, M.A. Ferenczi ${ }^{1}$

${ }^{1}$ Imperial College London, Molecular Medicine NHLI, London, UK; ${ }^{2}$ Myocardial Function, NHLI, London, UK

Non-linear optical microscopy has been shown to be a powerful tool for non-invasive imaging of thick specimen. In particular two-photon excitation microscopy (TPE) and second harmonic signal generation (SHG) allow high-resolution imaging at increased penetration depths with minimal out-of-focus excitation light, and hence intrinsic optical sectioning (Chen X. et al. NatProtoc 2012). Furthermore no sample staining is needed, thus removing potential sources of artefact, because numerous endogenous fluorophores can be excited by a two-photon mechanism. Second harmonic signals are generated in non-centrosymmetric structures such as collagen (Williams R. et al. BiophysJ.2005). The combination of these two techniques provides a new tool for diagnosis of many diseases (Han M. et al. OptExpress 2005; Sun W. et al. J BiomedOpt 2008; Lacomb, R. et al. Biophys J. 2008). Many cardiac diseases have been associated with increased fibrosis and changes in the organization of fibrillar collagen (Schenke-Layland K. et al. EurHeartJ 2009; Tsai M. et al. JBiomedOpt 2010). We combine the use TPE (to visualize endogenous fluorophores like elastin and myocyte proteins) with the simultaneous collection of SHG from collagen fibrils. We quantitatively analyse TPE and SHG signals by intensity-based and volumetric methods to evaluate the increase of fibrosis in infarcted (rat model of myocardial infarction through proximal coronary ligation, Lyon A.R. et al. P NATL ACAD SCI 2009) and healthy cardiac trabeculae. An $80 \%$ increase in collagen fibrosis is measured 20 weeks after infarction as well as a different spatial distribution within the volume providing insight on the morphological effect of disease progression. 


\section{1}

Lysine methyltransferase Smyd2 controls sarcomere organization and function by recruiting Hsp90 to titin

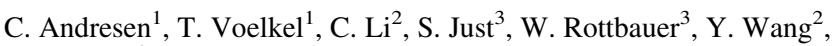
S.T. Smale ${ }^{2}$, W.A. Linke ${ }^{1}$

${ }^{1}$ Ruhr University Bochum, Department of Cardiovascular Physiology, Germany; ${ }^{2}$ University of California, Los Angeles, USA; ${ }^{3}$ Department of Internal Medicine III, Ulm University, Germany

Lysine methylation of (histone) proteins controls gene expression and DNA repair in the nucleus; however, this type of posttranslational modification also occurs in the cytoplasm, but is little understood in terms of its functional role. The lysine methyltransferase Smyd2 was recently shown to methylate the cytoplasmic chaperone Hsp90 in striated muscle cells (Donlin et al., Genes Dev, 2012; DOI: 10.1101/gad.177758.111***). This modification promotes the interaction of a Smyd2-Hsp90 complex with the N2A-domain of the giant sarcomere protein titin.

Aiming at elucidating the role of Smyd2 in myocardium, we demonstrated Smyd 2 expression in the cytoplasm of human, rodent, and chicken cardiomyocytes. Overexpressed Smyd2-GFP or endogenous Smyd2 associated with the sarcomeric I-band region in cultured cardiomyocytes and co-localized with the titin N2A-domain. Binding between human N2A-titin domain and Smyd2 was confirmed in yeast-two-hybrid and GST-pulldown assays. Within the N2A-domain the binding site was mapped to a unique sequence encoded by human titin-exon 104. Within Smyd2, short segments near the N-terminus and at the extreme $\mathrm{C}$-terminus were necessary for the interaction. Smyd2-knockdown in zebrafish using an antisense nucleotide morpholino approach caused a severe muscle and heart phenotype, the latter evident from a $\sim 50 \%$ reduction in fractional shortening at 72 h-post-fertilization. Despite the known interaction of Smyd2 with p53 and a small increase in p53-transcript levels in Smyd2-morpholino zebrafish, no alteration of apoptosis was detected in fish heart by TUNEL staining. Global knockout of Smyd2 in mice resulted in a significantly elevated heart/body weight ratio in males and a trend towards an increased ratio in females, at 6 months of age. Smyd3, 4 and 5, but not Smyd1, were slightly increased at the transcript level. Transverse aortic clipping did not aggravate the cardiac hypertrophic response, nor did it alter the ejection fraction.

These results suggest a role for Smyd2 in cardiac performance in both fish and rodents and also imply compensatory mechanisms for the Smyd2 gene in the hearts of higher vertebrates. Since Smyd2 helps recruit methyl-Hsp90 to the titin N2A-domain, where the chaperone exerts a protective effect, we conclude that, as in skeletal muscle, the formation of a protein complex between methyl-Hsp90, Smyd2 and the titin I-band domain is important for maintaining myofilament organization and function in myocardium.

\section{2}

\section{Small heat shock proteins associate with the titin springs and protect them from aggregation}

\section{S. Kötter ${ }^{1}$, L. Nagel-Steger ${ }^{2}$, P. Lang ${ }^{1}$, W.A. Linke ${ }^{1}$}

${ }^{1}$ Ruhr University Bochum, Department of Cardiovascular Physiology, Germany; ${ }^{2}$ Heinrich Heine University, Department of Physical Biology, Düsseldorf, Germany

The I-band portion of the giant muscle protein titin is elastic, but detailed knowledge of titin arrangement and interactions is lacking. As the titin springs are constantly exposed to mechanical stress, it can be expected that protein quality control mechanisms are active on titin to protect its mechanical function. We hypothesized that the small heat shock proteins (sHSPs), alpha-B-crystallin and HSP27, have protective effects on the elastic titin region.

We first determined whether or not select domains from the elastic titin region can associate with one another, as observed for titin's "distal" immunoglobulin domain-like (Ig-)region. Using sedimentation-velocity centrifugation and size-exclusion chromatography of purified human titin fragments, we detected a monomeric state of the titin N2B-domain or the N2B-unique sequence contained therein. The constitutively expressed PEVKdomain and the Ig-only fragment I9-I12 also behaved as monomers in vitro, whereas the N2A-segment showed both dimeric and monomeric behavior. Yeast-2-hybrid and GST-pulldown interaction tests demonstrated that both the N2B and the N2A domain of titin, but not the PEVK-fragment, bind to both alphaB-crystallin and HSP27. These interactions were confirmed on isolated human cardiac or rabbit psoas myofibrils incubated ex vivo with either sHSP type. Stretching myofibrils to promote titin-domain unfolding increased the propensity of HSP27 to bind I-band titin. Chemically unfolded (using $8 \mathrm{M}$ urea) titin domains were probed for their propensity to aggregate, and the N2A-segment but not the N2Bdomain indeed showed aggregation under acidic conditions $(\mathrm{pH}$ 6.7), whereas at $\mathrm{pH} 7.2$ no fragment aggregated. Importantly, alphaB-crystallin prevented the aggregation of the $\mathrm{N} 2 \mathrm{~A}$-segment at $\mathrm{pH}$ 6.7 partially (molar ratio of N2A:alphaB-crystallin, 1:5) or fully (ratio, 1:10); HSP27 did not have this protective effect. In cultured embryonic rat cardiomyocytes, the two sHSPs translocated from the cytosol to the sarcomeric Z-disk/I-band region on inhibition of the proteasome. In adult rat cardiomyocytes both sHSPs associated with the I-band titin already under normal culture conditions.

We conclude that (1) titin filaments may run through the elastic segment mainly as monomers; (2) sHSPs associate with elastic titin domains under various stress conditions; and (3) alphaB-crystallin is able to protect I-band titin regions from aggregation under adverse intracellular circumstances.

173

Deranged myofilament phosphorylation and function in experimental heart failure with preserved ejection fraction

N. Hamdani ${ }^{1}$, K.G. Bishu ${ }^{2}$, M.M. Redfield ${ }^{2}$, W.A. Linke ${ }^{1}$

Ruhr University Bochum, Department of Cardiovascular Physiology, Germany; ${ }^{2}$ Mayo Clinic and Foundation, Rochester MN, USA

Heart failure with preserved ejection fraction (HFpEF) is a major cause of morbidity and mortality. Key pathophysiological alterations in HFpEF include increased left ventricular (LV) stiffness and abnormal relaxation, resulting in impaired LV filling. We hypothesized that changes in phosphorylation and function of myofilament proteins occur in experimental HFpEF vs. normal myocardium studied under two different conditions of tissue procurement, beatingheart biopsy and postmortem tissue sampling.

LV tissue samples were procured (beating-heart biopsy, $n=7$ / group; postmortem tissue, $n=7-9$ /group) from young or old normal dogs (CTRL) and old dogs made hypertensive by renal wrapping (OHT/HFpEF). We assessed expression and phosphorylation of myofilament proteins, expression/activity of major protein kinases (PK) and phosphatases (PP), myofilament calcium sensitivity $\left(\mathrm{pCa}_{50}\right)$ and passive tension $\left(\mathrm{F}_{\text {passive }}\right)$ of isolated permeabilized cardiomyocytes. Alterations in OHT vs. CTRL included decreases in the N2BA:N2B titin-isoform ratio, PKG activity and phosphorylation of cMyBPC, cTnT, all-cTnI, cTnI (S23/S24), all-titin and titin-N2Bus (S4010; S4099), but increases in PKC $\alpha$ activity, PP1 and PP2a expression, $\mathrm{F}_{\text {passive }}$, and phospho-PEVK (S11878) titin. $\mathrm{pCa}_{50}$ was increased in OHT-myocytes from beating-heart biopsies but lowered 
in those from postmortem tissue, concomitant with reduced cMLC-2 phosphorylation only in OHT biopsies.

This patient-mimicking HFpEF model shows titin stiffening owing to isoform shift and altered phosphorylation, all contributing to elevated LV stiffness as a common feature of HFpEF. Many regulatory myofilament proteins are hypophosphorylated and myofilament calcium sensitivity is increased, suggesting contractile dysregulation is an early step in HFpEF. Not all remodeling events seen in postmortem tissue were genuinely disease-related.

\section{4}

\section{Structural and functional characterisation of the interactome} of nebulin and nebulette $\mathrm{SH3}$ domains

M.C. Vidal-Pelissier ${ }^{1}$, Z. Peijian ${ }^{2}$, P. Kursula ${ }^{3}$, F. Lehman ${ }^{4}$, M. Wilmanns ${ }^{1}$, I. Kursula ${ }^{3}$

${ }^{1}$ EMBL, Hamburg, Germany; ${ }^{2}$ Institute of Structural Biology-HZ, Munich, Germany; ${ }^{3}$ CSSB-HZI and University of Hamburg, Hamburg, Germany; ${ }^{4}$ ETH, Zurich, Switzerland

Nebulin (Neb) is a very large filamentous protein $(600-900 \mathrm{kDa})$ tightly associated to the thin filament of the muscle sarcomere throughout its length. Nebulin is mostly found within the sarcomeres of skeletal muscles but was also identified at a low level in cardiac muscle cells. However, the sarcomeres of cardiac muscles predominantly contain a nebulin-like protein called nebulette (Net) which is a "short version" of nebulin (100 kDa), highly similar to its C-terminal part. Nebulin and nebulette both comprise a very C-terminal SH3 domain which is located in the Z-disk of the sarcomere, and both share the property of binding the proline-rich region of some Z-disk/I-band proteins. The interactome of the Neb/ Net SH3 domains is thought to play a key role for the proper function of these proteins by anchoring them within the Z-disk and linking them to signalling pathways involved in the sarcomere formation and maintenance. We have undertaken the structural and functional characterisation of this interactome in order to shed light on the specific properties of these complexes and decipher their role in the context of the sarcomere.

We present here the first X-ray structures of human nebulin and nebulette in an apo form, as well as complexes with peptides derived from their Z-disk binding partners.

176

The working stroke of muscle myosin studied in skinned fibres by recording the isotonic velocity transient and the effect of changes in concentration of inorganic phosphate

\section{Caremani, L. Melli, M. Dolfi, V. Lombardi, M. Linari}

University of Florence, Laboratory of Physiology DBE, Sesto Fiorentino, Italy

The coupling between chemical and mechanical steps of actomyosin ATPase cycle was studied in situ by using fast mechanical protocols in $\mathrm{Ca}^{2+-}$ activated demembranated fibres from rabbit psoas under sarcomere length control (sarcomere length $2.4 \mu \mathrm{m}$, temperature $12{ }^{\circ} \mathrm{C}$ ). Under these conditions it is possible to record the isotonic velocity transient following a stepwise drop in force from the isometric plateau force (Piazzesi et al. J Physiol 545:145, 2002) and investigate the effects of the concentration of inorganic phosphate (Pi) on both the early rapid shortening (phase 2), that is due to the synchronized working stroke in the actin attached motors, and the following reduction or pause in shortening velocity (phase 3 ), due to motor detachment preceding the steady state (phase 4) detachmentattachment (characteristic of the $T-V$ relation and power output).

With respect to control (no added $\mathrm{Pi}$ ), the increase of [Pi] to $10 \mathrm{mM}$ did not change the rate of early rapid shortening following the drop to the same fraction of isometric force, while reduced its size and made the subsequent pause of shortening briefer and the final shortening velocity higher. Steady state and transient mechanical responses were simulated with a kinetic-mechanical model of the actomyosin ATPase cycle that incorporates Huxley and Simmons mechanism of force generation. The data could be predicted only if myosin motors at an intermediate stage of both the working stroke and product release can slip to the next Z-ward actin monomer.

Supported by MIUR, Ministero della Salute and Ente Cassa di Risparmio di Firenze (Italy).

\section{7}

The source of non-linearity in the elasticity of the muscle half-sarcomere

L. Fusi, E. Brunello, M. Reconditi, G. Piazzesi, V. Lombardi

University of Florence, Laboratory of Physiology DBE, Sesto Fiorentino, Italy

Recent claims that the mechanical estimate of the myosin motor stiffness in a contracting muscle fiber is influenced by the contribution of weakly bound myosin heads (Colombini et al. Biophys $J$ 98:2582, 2010 ) or by non-linearity of filament compliance (Mansson Biophys $J$ $99: 1869,2010$ ) have brought into question the high stiffness of the myosin motor $\left(\sim 3 \mathrm{pN} \mathrm{nm}^{-1}\right)$, determined using single fibre sarcomere-level mechanics and X-ray diffraction (Decostre et al. Proc Natl Acad Sci USA 102:13927, 2005; Piazzesi et al. Cell 131:784, 2007).

We applied $4 \mathrm{kHz}$ length oscillations of $\sim 1 \mathrm{~nm}$ per half-sarcomere to single fibres isolated from the tibialis anterior muscle of Rana esculenta $\left(4{ }^{\circ} \mathrm{C}\right.$, sarcomere length $\left.2.15 \mu \mathrm{m}\right)$ to determine the sarcomere strain-force relation during the development of isometric force $\left(T_{0}\right)$ and during the force redevelopment from zero following the end of $2.5 \%$ unloaded shortening. The results confirm both the $\sim 3 \mathrm{pN} \mathrm{nm}^{-1}$ motor stiffness and the linearity of the filament compliance in the whole range of forces and show that the non-linearity in the sarcomere strain-force relation at forces $<0.3 T_{0}$ is due to a transient additional elastic component in parallel with the force-generating myosin motors. At the start of force development the stiffness of this component is $\sim 5 \%$ of that of the motor array at $T_{0}$, while at the start of force redevelopment following unloaded shortening the corresponding value is $\sim 25 \%$. The nature of this elasticity in parallel with forcegenerating myosin motors is discussed.

Supported by MIUR, Ente Cassa di Risparmio di Firenze and IIT-SEED (Italy).

\section{8}

A new theory on $k_{\text {tr }}$ and $k_{\text {act }}$ with series elastic elements

\section{Kawai}

Department of Anatomy and Cell Biology, University of Iowa, Iowa City, IA 52242, USA

It has been known for some time that when the length of active muscle fiber is released suddenly by $10-20 \%$ and restretched to the original length, the tension falls close to 0 with the length release, then tension redevelops exponentially with the rate constant $k_{\mathrm{tr}}(1)$. Similarly, when the bathing solution is switched from relaxing solution to activatng solution in myofibrils (9), a similar tension time course is observed with the rate constant $k_{\text {act, }}$ where $k_{\text {tr }} \sim k_{\text {act }}$. The rise of tension has been interpreted to represent the force generation step (1), i.e., a partial cross-bridge cycle. Here I propose a model, in which cross-bridges cycle many times in this period by stretching 
series elastic elements. One assumption needed is that either the step size $(\eta)$ or the stepping rate $(v)$ decreases linearly with tension $(F)$ :

$\eta=\eta_{0}\left(1-F / F_{0}\right)$

or

$v=v_{0}\left(1-F / F_{0}\right)$

where $F_{0}$ is isometric tension, and $F=F(t)$ is tension transient $\left(F \leq F_{0}\right) ; \eta_{0}$ is step size and $v_{0}$ is the number of steps in $1 \mathrm{~s}$ in the absence of load. There is experimental evidence for Eq. 1 (8), and Eq. 2 represents the Fenn effect. Distance traveled by cross-bridges in time $d t$ is $\eta v d t$, which stretches series elastic. Thus, the increase in tension $(d F)$ is the product of stiffness $(\sigma)$ of the series elastic and the distance traveled: $d F=\sigma v \eta d t$. By using Eq. 1 or 2 (not both), we get:

$d F / d t+k F=k F_{0}$

where $k \equiv \sigma \eta_{0} v_{0} / F_{0}$, hence

$F_{0} / \sigma=\eta_{0} v_{0} / k$

By solving Eq. 3 with the initial condition $F(0)=0$,

$F(t)=F_{0}[1-\exp (-k t)]$

results. Thus, this system has one exponential process with the rate constant $k$. Eq. 5 is a good approximation for describing the available time course data $(1,9-11)$ which experimentally measured $k_{\text {tr }}$ and/or $k_{\text {act. }}$.

$1 / v_{0}$ is the average time a cross-bridge spends for one cycle, and is related to the elementary steps by:

$\frac{1}{v_{0}}=\frac{1}{\lambda_{2}}+\frac{1}{\lambda_{3}}+\frac{1}{k_{6}+k_{-6}} \approx \frac{1}{k_{6}}$

In Eq. 6, each term in the middle corresponds to a time constant: $1 / \lambda_{2}$ is that of phase 2 , which is the average time a cross-bridge spends to complete detachment of the myosin head from actin; $1 / \lambda_{3}$ is the average time the cross-bridge spends to complete force generation step (time constant of phase 3 ), and $1 /\left(k_{6}+k_{-6}\right)$ is the average time the cross-bridge spends to complete ADP isomerization step that lead to ADP release (known as the "ADP release" step). The approximation of Eq. 6 is based on $k_{6} \ll k_{6} \ll v_{3}<v_{2}$ (5), hence $v_{0} \approx k_{6}$ is the $1^{\text {st }}$ approximation. We reported that $k_{6} \cong 16-18 \mathrm{~s}^{-1}$ in rabbit psoas fibers at $15-20{ }^{\circ} \mathrm{C}(13)$; The step size of $\eta_{0}=5.3 \mathrm{~nm}$ was reported (6) (see also $(3,7)$ ), and $k_{\mathrm{tr}}=8.0 \mathrm{~s}^{-1}$ and $k_{\mathrm{act}}=7.9 \mathrm{~s}^{-1}$ were reported (9). Thus, the right side of Eq. 4 is:

$$
\eta_{0} v_{0} / k=5.3 \mathrm{~nm} \times 17 \mathrm{~s}^{-1} / 7.95 \mathrm{~s}^{-1}=11 \mathrm{~nm} .
$$

The left side of Eq. $4\left(F_{0} \rho\right)$ is the same as the (force):(stiffness) ratio, or the instantaneous length release needed to abolish full tension. We reported (Tension):(elastic modulus) $=1.14 \%$ in rabbit psoas fibers at $15{ }^{\circ} \mathrm{C}$ (13). Therefore,

$($ Force $) /($ stiffness $)=1.25 \mu m \times .0114=14 \mathrm{~nm}$

where $1.25 \mu \mathrm{m}$ is the half sarcomere length. The value given in Eq. 7 can be somewhat less than this, because $1.14 \%$ was based on an extrapolation with a truncation of high frequency data. Thus, an approximate agreement can be reached for both sides of Eq. 4, which justifies this equation and the theory behind it. The above analy-sis provides support for the hypothesis that $k_{\mathrm{tr}}$ is limited by the slowest step in the cross-bridge cycle (2). The fact that $k_{\text {tr }}$ is affected by Pi $(10,12)$ may be related to the fact that $\lambda_{3}$ is sensitive to the phosphate concentration $(4,5)$ as the 2 nd approximation.

\section{References}

[1] Brenner B, Eisenberg E (1986) Proc Natl Acad Sci USA. 83:3542-3546.
[2] Gordon AM, Homsher E, Regnier M (2000) Physiol. Rev. 80:853-924.

[3] Guilford WH, Dupuis DE, Kennedy G, Wu J, Patlak JB, Warshaw DM (1997) Biophys J. 72:1006-1021.

[4] Kawai M (1986) J Muscle Res Cell Motil. 7:421-434.

[5] Kawai M, Halvorson HR (2007) J Muscle Res Cell Motil. 28:371-395.

[6] Kitamura K, Tokunaga M, Iwane AH, Yanagida T (1999) Nature. 397:129-134.

[7] Molloy JE, Burns JE, Kendrick-Jones J, Tregear RT, White DC (1995) Nature. 378:209-212.

[8] Piazzesi G, Reconditi M, Linari M, Lucii L, Bianco P, Brunello E, Decostre V, et al. (2007) Cell. 131:784-795.

[9] Piroddi N, Tesi C, Pellegrino MA, Tobacman LS, Homsher E, Poggesi C (2003) J Physiol. 552:917-931.

[10] Regnier M, Morris C, Homsher E (1995) Am J Physiol. 269:C1532-1539.

[11] Stehle R, Kruger M, Scherer P, Brixius K, Schwinger RH, Pfitzer G (2002) Basic Res Cardiol 97.1:I127-135.

[12] Tesi C, Colomo F, Nencini S, Pirodi N, Poggesi C (2000) Biophy J. 78:3081-3092.

[13] Zhao Y, Kawai M (1994) Biophys J. 67:1655-1668.

\section{0}

\section{Uncoupling of stress from myosin light chain (RLC) phosphorylation during cyclic nucleotide induced relaxation of smooth muscle revisited}

G. Pfitzer ${ }^{1}$, S. Puetz ${ }^{1}$, M.S. Hunger ${ }^{1,2}$, L.T. Lubomirov ${ }^{1}$, H. Piechura ${ }^{3}$, L. Reimann ${ }^{3}$, B. Warscheid ${ }^{3}$, M.M. Schroeter ${ }^{1}$

${ }^{1}$ University of Cologne, Institute of Vegetative Physiology, Germany; ${ }^{2}$ University of Cologne, Clinics for Anaesthesiology and Surgical Intensive Care, Germany; ${ }^{3}$ University of Freiburg, Faculty of Biology and BIOSS Centre for Biological Signalling Studies, 79104 Freiburg, Germany

According to the phosphorylation theory of smooth muscle (sm) contraction, relaxation of sm should be preceded by dephosphorylation of RLC at Ser19, the site considered to be responsible for regulation of contraction. However, cAMP/cGMP induced relaxation was frequently uncoupled from RLC dephosphorylation giving rise to the notion that cyclic nucleotide mediated relaxation involves mechanisms independent of RLC dephosphorylation. Yet it is unclear whether this involves pSer19 or one of the other phosphorylation sites of RLC including Thr8 and Thr18. Therefore, we monitored pSer19 with Western blots in mouse tail arteries and gastric fundus during respectively urocortin/cAMP and NO induced relaxation.

Urocortin induced relaxation of arterial sm was associated with dephosphorylation of pSer19 as was relaxation of alpha-toxin permeabilized arteries ( $\mathrm{pCa}$ 6.1) induced by selective activators of the cAMP targets, PKA and exchange proteins activated by cAMP (EPACs). In a similar manner, NO mediated relaxation of intact gastric fundus was associated with dephosphorylation of pSer19. In contrast, phosphorylation of pThr18 was elevated during sustained relaxation gastric fundus as indicated by LC/MS and Western blots analysis. We have no evidence for phosphorylation of the N-terminal PKC phosphorylation sites (Ser1/2 and Thr8) nor for Thr18/Ser19 diphosphorylation.

In conclusion, our results indicate that the phosphorylation rebound during sustained relaxation of gastric fundus is attributable to monophosphorylation of pThr18 whereby the functional relevance is currently not known. Based on our results we propose that cyclic nucleotide induced relaxation is mediated by dephosphorylation pSer19 in keeping with the phosphorylation theory of smooth muscle contraction. 


\section{1}

Interplay between Ankrd2, Akt/Gsk3b and NF-kB pathways during myogenic differentiation

N.K. Verma ${ }^{1}$, D. Yamamoto $^{2}$, M.L. Bang ${ }^{2,3,4}$, G. Lanfranchi ${ }^{1}$, C. Bean ${ }^{1}$

${ }^{1}$ University of Padova, Department of Biology, Padova, Italy; ${ }^{2}$ Dulbecco Telethon Institute, Italy; ${ }^{3}$ Institute of Genetic and Biomedical Research, Milan Unit, National Research Council (IRGBCNR), Milan, Italy; ${ }^{4}$ Humanitas Hospital, Rozzano (Milan), Italy

The overexpression of Ankrd2 in skeletal muscle in vivo suggests that this factor may significantly contribute to physiological and pathological muscle remodeling, moving from the titin-telethonin-MLP stretch sensor complex towards the nucleus. In addition, we have demonstrated that the overexpression of Ankrd2 in proliferating myoblasts severely compromises the differentiation program by affecting cell death pathways. To achieve its functions, Ankrd2 is involved in multiple interactions with both structural and regulatory proteins, displaying dynamic distributions within cells. Thus, we explored the functional role of Ankrd2, especially in regard to its interplay with other pathways.

A genomic approach based on microarray technology was used to identify the gene networks that are disturbed in Ankrd2 knockout or overexpressing primary cells during differentiation. Our data strengthen the fact that Ankrd2 is a potent regulator in muscle cells, affecting a multitude of pathways and processes. An interesting result reveals that Ankrd2, NF-kB and Akt/Gsk3b signaling pathways can converge in regulating muscle differentiation and the functional dissection of these signaling cascades shows that Ankrd2 overexpression inhibits both Gsk3b and NF-kB activity. We have new evidence showing that Ankrd2 associates with the transcriptionally inactive NF-kB member $\mathrm{p} 50$ in the nucleus, thereby interfering with NF-kB-induced transcriptional regulation. In particular, Ankrd2 represses the transcription of Gsk3b by formation of a p50/Ankrd2 complex that binds to the specific $\mathrm{kB}$ site of the Gsk3b promoter. Finally we show that NF-kB represses Ankrd2 transcription by a negative feedback loop. Thus, the interplay between these pathways reveals novel molecular mechanisms regulating muscle development.

Sponsored by the participating research network MUZIC.

182

Rapid changes in free mitochondrial calcium concentration in fast skeletal muscle fibers from wild type and calsequestrin null mice

M. Scorzeto ${ }^{1}$, M. Giacomello ${ }^{2}$, L. Toniolo ${ }^{1}$, M. Canato ${ }^{1}$, C. Paolini ${ }^{3,4}$, F. Protasi ${ }^{3,4}$, C. Reggiani ${ }^{1,4}$, G.J.M. Stienen ${ }^{5}$

${ }^{1}$ Department of Human Anatomy and Physiology, University of Padova, Padua, Italy; ${ }^{2}$ Department of Experimental Veterinary Sciences, University of Padova, Padua, Italy; ${ }^{3} \mathrm{CeSI}-$ Centro Scienze dell'Invecchiamento, Dept. of Neuroscience and Imaging, University G. d'Annunzio, Chieti, Italy; ${ }^{4}$ IIM Interuniversity Institute of Myology, Italy; ${ }^{5}$ VU University Medical Center, Institute for Cardiovascular Research, Laboratory for Physiology, Amsterdam, The Netherlands

Mitochondrial $\mathrm{Ca}^{2+}$-uptake is important for the regulation of aerobic ATP production and is involved in apoptosis. Muscle fibers lacking calsequestrin (CASQ) present alterations in sarcoplasmic reticular calcium content and mitochondrial morphology, which may affect cellular calcium homeostasis and mitochondrial metabolic function.

Experiments were performed in enzymatically dissociated FDB muscle fibers from wild type (WT) and CASQ-null mice.
Mitochondrial $\mathrm{Ca}^{2+}$-handling was measured using a ratiometric FRET-based indicator ( $\mathrm{mtD} 3 \mathrm{cpv}$ Cameleon) targeted to the mitochondrial matrix. Experiments were conducted in the presence $(1 \mathrm{mM})$ and absence of extracellular calcium. Mitochondrial membrane potential was determined using TMRM. Mitochondrial density and morphology was studied by electron microscopy.

Small but significant differences in free mitochondrial $\mathrm{Ca}^{2+}$-concentration $\left(\left[\mathrm{Ca}^{2+}\right]_{\text {mito }}\right)$ were observed between quiescent WT and CASQ-null fibers. The free $\left[\mathrm{Ca}^{2+}\right]_{\text {mito }}$ during steady state electrical stimulation at $1 \mathrm{~Hz}$ showed a rapid increase with a $10-90 \%$ rise time of $18.4 \pm 0.4 \mathrm{~ms}$. The decline in $\left[\mathrm{Ca}^{2+}\right]_{\text {mito }}$ during and after stimulation trains was governed by 3 temporally distinct processes with rate constants of approximately $40 \mathrm{~s}^{-1}, 1.6 \mathrm{~s}^{-1}$ and $0.2 \mathrm{~s}^{-1}$ (at $26^{\circ} \mathrm{C}$ ). During sustained contractions in WT fibers, frequency-dependent increases in free $\left[\mathrm{Ca}^{2+}\right]_{\text {mito }}$ occurred, which were smaller in the absence than in the presence of external $\mathrm{Ca}^{2+}$. In CASQ-null fibers the increases in free $\left[\mathrm{Ca}^{2+}\right]_{\text {mito }}$ was less pronounced.

These results provide direct evidence for rapid $\mathrm{Ca}^{2+}$ uptake by the mitochondria and suggest that the mitochondrial $\mathrm{Ca}^{2+}$ uptake is sensitive to the amount of $\mathrm{Ca}^{2+}$ available in intracellular (notably the sarcoplasmic reticulum) and extracellular stores.

\section{4}

Interactions between sarcoplasmic reticulum $\mathrm{Ca}^{2+}$ handling and mitochondria in skeletal muscle

\section{H. Westerblad}

Karolinska Institutet, Department of Physiology \& Pharmacology, Stockholm, Sweden

Mitochondria are of fundamental importance in skeletal muscle because they determine the oxidative capacity and hence the fatigue resistance of muscle fibers. Mitochondrial signaling also has general effects on muscle function, e.g. by altering gene transcription and by inducing apoptotic signaling. Recent studies from our laboratory show that $\mathrm{Ca}^{2+}$ plays an essential role in the interaction between mitochondria and general muscle function. In this context the concentration and location of $\mathrm{Ca}^{2+}$ is critical for the physiological effects.

We have shown that a minor increase in the $\mathrm{Ca}^{2+}$ leak from the sarcoplasmic reticulum (SR) is accompanied by increased mitochondrial biogenesis and increased fatigue resistance. On the other hand, a larger SR $\mathrm{Ca}^{2+}$ leak results in muscle weakness. Moreover, muscle fibers of mice with mitochondrial myopathy show that increased $\mathrm{Ca}^{2+}$ concentration in the mitochondria is accompanied by decreased SR $\mathrm{Ca}^{2+}$ content and muscle weakness; pharmacological treatment to reduce this mitochondrial $\mathrm{Ca}^{2+}$ load leads to improved muscle function. These complex $\mathrm{Ca}^{2+}$-dependent interactions will be discussed.

\section{6}

\section{Sarcopenia and inflamm-aging}

C. Franceschi

Dept. of Experimental Pathology and CIG-Interdepartmental Center "L. Galvani”, University of Bologna, Bologna, Italy

Aging is a very complex phenomenon that is still not completely understood. In the last years, it has been observed that aging is characterised by an imbalance between inflammatory and antiinflammatory networks, which results in a chronic low-grade inflammatory status that we proposed to call Inflamm-aging. 
Inflamm-aging is a driving force for several age-related diseases as well as changes in body composition. These changes are characterized by a relative decline of muscle mass and an associated increase of fat mass. This condition is termed sarcopenia and represents an important risk factor for disability, loss of autonomy, morbidity and mortality in the elderly. Many factors are involved in the etiology of sarcopenia, among which muscle disuse, malnutrition, low production of hormones and an unbalanced rate of protein degradation/synthesis. Other factors likely affecting sarcopenia are apoptosis, ROS production and impaired energy metabolism. Many studies indicate that several molecular signalling pathways are involved in the development of sarcopenia, among which those of p53, NF-kappaB and Grp75, all of them affected by inflammation. Contrasting data on the role of inflammatory mediators such as IL-6 in muscle metabolism are present. An increased level of IL-6 is associated with low mobility and muscle force, but at the same time IL-6 appears to be a trophic factor for muscles. Within this puzzling scenario, the relationships between inflamm-aging and the molecular mechanisms of sarcopenia remain unclear. Available data and evidence on this topic will be discussed and hypotheses will be critically reviewed.

Supported by the EU Framework 7 Project MYOAGE: Understanding and Combating age related muscle weakness Grant Agreement Number: 223576.

\section{7}

Short-term expression profiling of electrically stimulated muscle reveals the initial points of fast to slow transition

G. Busolin ${ }^{1}$, F. Chemello ${ }^{1}$, C. Bean ${ }^{1}$, B. Blaauw ${ }^{2}$, P. Cancellara ${ }^{2}$, P. Laveder ${ }^{1}$, C. Reggiani ${ }^{2}$, G. Lanfranchi ${ }^{1}$

${ }^{1}$ University of Padua, Department of Biology, Padua, Italy; ${ }^{2}$ University of Padua, Department of Biomedical Science, Padua, Italy

Skeletal muscle fibers have a remarkable capacity to adjust their molecular, functional, and metabolic properties in response to developmental and environmental stimuli. An understanding of the cross talk between different signaling pathways that control muscle fiber plasticity has a significant impact on the study of muscle physiology and pathology. Chronic low-frequency stimulation (CLFS), mimicking the impulse pattern normally delivered to slowtwitch muscle, changes the metabolic and contractile properties of fast-twitch muscles. So far, studies have focused on the effects produced after days or weeks of stimulation.

We present the first microarray study that identifies genetic programs activated at the earliest stages of this process. In order to reduce biological noise caused by different cell types, we applied here microgenomic analyses at the level of single, isolated fibers. The fast EDL muscle was subjected to CLFS for 6 and $12 \mathrm{~h}$. At each time point, the expression profiles from 10 stimulated fibers were compared with those obtained from 10 unstimulated fibers. Several putative master switch genes were identified. In particular, more than a thousand genes result differentially expressed after $12 \mathrm{~h}$ of stimulation, and most of them are involved in regulation of transcription. Furthermore, pathway analysis reveals that several differentially expressed genes are involved in metabolic and signal transduction pathways, in particular insulin, mTOR and MAPK signaling.

The challenge now is to understand how early transcriptional changes can modify those pathways and how they interact each other, in order to adapt metabolic and contractile properties to the new physiological demand.

Busolin is a candidate for a young investigator award to be judged during the EMC 2012.
188

How much do myhc isoform $m R N A$ levels correlate with myhc protein levels and fiber type composition?

T. Soukup ${ }^{1}$, J. Žurmanová ${ }^{1,2}$

${ }^{1}$ Institute of Physiology, (v.v.i.), AS CR, Czech Republic; ${ }^{2}$ Faculty of Science, Charles University, Prague, Czech Republic

It is generally accepted that skeletal muscle fiber types are defined by MyHC isoform composition at protein level, which is dependent on the specific $m R N A$ content. Our goal was to demonstrate these relations in the slow soleus and fast extensor digitorum longus (EDL) muscles of adult euthyroid, hyperthyroid and hypothyroid inbred Lewis strain rats.

MyHC $m R N A$ expression was analyzed by quantitative real time RT-PCR, MyHC protein isoforms by SDS-PAGE and muscle composition using the fiber type immunohistochemical analysis.

Comparison of the results from different measurements showed for both muscles equivalent composition in percent of $\mathrm{MyHC}$ in the case of gene transcripts $(m R N A-1,-2 \mathrm{a},-2 \mathrm{x} / \mathrm{d},-2 \mathrm{~b})$, protein isoforms (MyHC-1, -2a, -2x/d, -2b) and fiber types (type 1, 2A, 2X/D, 2B). All parameters changed accordingly with the altered thyroid status.

We present quantitative evidence of direct correlation for previously presumed relationship between $m R N A$ level, protein content and fiber type composition and we suggest that real time RT-PCR can be used as a routine method wherever analysis of muscle composition changes is needed. If the same correlation will be proven in humans, it could be successfully used for muscle biopsies, as real time RT-PCR requires much smaller samples compared to other two methods.

Supported by GAČR 304/08/0256, MSM0021620858 grants and the Research project AV0Z 50110509.

\section{9}

Drosophila $\alpha B$-crystallin orthologue lethal (2) essential for life is required for muscle shaping and for proper sarcomere organisation

I. Wójtowicz ${ }^{2,1}$, M. Daczewska ${ }^{2}$, K. Jagla ${ }^{1}$, T. Jagla ${ }^{1}$

${ }^{1}$ GReD, INSERM U931, University of Clermont Ferrand, 28, Place Henri Dunant, 63000 Clermont-Ferrand, France; ${ }^{2}$ Department of Animal Developmental Biology, Zoological Institute, University of Wroclaw, Sienkiewicza 21, 50-335 Wrocław, Poland

The gene lethal (2) essential for life (lefl), the Drosophiila orthologue of $\alpha B$-crystallin involved in Desminopathies in human, codes for small heat shock protein with chaperon activity. left is expressed during Drosophila development in embryonic, larval and adult somatic and heart muscles. We investigated the role of lefl during embryonic myogenesis and during sarcomeres assembly in larva by analyzing muscle-specific gain and loss of its function using GAL-4/ UAS system. Lefl protein displays muscle-specific expression in late embryos and accumulates in a dotty pattern close to the muscle cell membranes. Its loss and gain of function phenotypes indicate it is involved in regulating embryonic muscle shapes by interacting with F-actin network. To our knowledge lefl is the first gene acting as shape regulator in developing muscles. In muscles of 3rd instar larvae Lefl protein is mainly located on Z-disc and $\mathrm{M}$ line extensions linking sarcomeres with muscle membrane. It co-localizes with Vimentin-like and Lamin C proteins, which like Desmin in vertebrates, are intermediate filament proteins. Under attenuation of lefl expression a number of perturbations of muscle pattern occur, like splitting or incorrect muscle attachment. Moreover, it has been noticed that muscles of lefl-deficient larvae are shorter and narrower with smaller number o nuclei and sarcomeres. Ultrastuctural analyses confirmed altered sarcomeres organization showing mitochondria with barely 
visible mitochondrial crests and increased amount of glycogen between myofilaments. We also demonstrated that muscle-specific lefl knockdown causes altered arrangement of Vimentin-like protein indicating affected intermediate filament structure. We observed a meshwork-like organization of Vimentin-labeled filaments on the surface of myofibrils indicating that LEFL and Vimentin-like proteins interact. Thus, the mis-arrangement of intermediate filaments observed in muscles with attenuated lefl expression appears reminiscent of defects in human Desminopathies caused by mutations in $\alpha B$-crystallin gene. We have expressed in Drosophila muscle cells Lefl protein with mutation R120G, present in $\alpha B$-crystallin gene causing Desmin Related Myopathy. Observed phenotypes were characterized by dislocation of nuclei, disorganization of Vimentinelike meshwork and formation of aggregates composed of Lefl and Vimentin-like proteins suggesting loss of proper interlink between those proteins. Altogether our data provide evidence that Lefl is an important element of myogenic pathway acting to shape embryonic muscles and to ensure proper sarcomeric organization in differentiated muscle fibers. Our data also reveal that intermediate filaments are part of sarcomeric structures in Drosophila muscles and support a view that fruit fly can be used as a model to study the genetic determinants of intermediate filaments linked myopathies.

\section{1}

\section{Structural studies of the Z-disc complex FATZ: alpha-actinin-2}

C.A. Rodriguez ${ }^{1}$, A Lehner ${ }^{1}$, J. Neuhold ${ }^{1}$, D. Mullerova ${ }^{2}$, N. Pinotsis ${ }^{1}$, E. De Almeida Ribeiro ${ }^{1}$, K. Djinović-Carugo ${ }^{1}$

${ }^{1}$ Max F. Perutz Laboratories, University of Vienna, Department of Structural and Computational Biology, Campus Vienna Biocenter 5, A-1030 Vienna, Austria; ${ }^{2}$ Evitra AB. Strandvägen 5A, S-11451 Stockholm, Sweden

The Z-disc defines the boundaries of the sarcomere which is the basic contractile unit of the striated muscle. The major Z-disc function is the cross-linking of thin filaments; besides, it hosts a sophisticated network of protein-protein interactions that supports stability and homeostasis in the myofibril. How this protein-protein interaction network is structurally organized to support the Z-disc architecture and function is unknown at molecular level. FATZ/calsarcin/myozenin, one of the nodes of this network, is a protein family of three members with several binding partners in the Z-disc. Among those is $\alpha$-actinin-2, which is the major component of Z-disc, cross-links actin filaments and interacts with a number of partners. The complex FATZ: $\alpha$-actinin- 2 appears in the early stages of myofibrilogenesis and it is suggested that FATZ acts as an adaptor protein in recruiting other proteins to the Z-disc.

Here we present a structural and biochemical characterization of the complex FATZ: $\alpha$-actinin-2, with the aim of providing a 3D model of the complex and to inform on the stability of this interaction. Our preliminary data suggest that FATZ-1 lacks defined tertiary structure. Nevertheless, it has the capacity of binding to the dimeric $\alpha$-actinin- 2 (1:2 molar ratio). Currently, we are determining the binding constants of this association and studying the structure and dynamics of FATZ1 upon binding to $\alpha$-actinin- 2 by means of nuclear magnetic resonance, small angle X-ray scattering and chemical cross-linking.

\section{3}

miRNAs mediate estrogen's impact on skeletal muscle-a study with postmenopausal monozygotic co-twins

V. Kovanen ${ }^{1,2}$, F. Olivieri ${ }^{3,4}$, M. Ahtiainen ${ }^{1,2}$, R. Lazzarini ${ }^{3}$, M. Capri ${ }^{5}$, M. Lorenzi ${ }^{6}$ E. Pöllänen ${ }^{1,2}$, C. Albertini $^{7}$, S. Salvioli $^{5}$, M. Alen ${ }^{8}$, U. Kujala ${ }^{2}$, G. Borghetti ${ }^{3}$, L. Babini ${ }^{3}$, J. Kaprio ${ }^{9}$, C. Franceschi ${ }^{5}$, S. Sipilä ${ }^{1,2}$, A.D. Procopio ${ }^{3,4}$
${ }^{1}$ University of Jyväskylä, Gerontology Research Centre, Jyväskylä, Finland; ${ }^{2}$ University of Jyväskylä, Dept of Health Sciences, Jyväskylä, Finland; ${ }^{3}$ Università Politecnica delle Marche, Division of Pathology, Dept Clinical and Molecular Sciences, Ancona, Italy; ${ }^{4}$ Advanced Technology Center forAging Research, Dept Clinical Pathology and Innovative Therapy, Scientific Technological Area INRCA-IRCCS, Ancona, Italy; ${ }^{5}$ University of Bologna, Dept Experimental Pathology, Bologna, Italy; ${ }^{6}$ Università Politecnica delle Marche, School of Medicine, Division of Neuroscience and Cell Biology, Dept Experimental and Clinical Medicine, Ancona, Italy; ${ }^{7}$ Università degli Studi di Urbino "Carlo Bo", Dipartimento di Scienze Biomolecolari, Sezione di Biochimica e Biologia molecolare, Urbino, Italy; ${ }^{8}$ University of Oulu, Dept Medical Rehabilitation, Oulu University Hospital and Institute of Health Sciences, Oulu, Finland;

${ }^{9}$ University of Helsinki, Dept Public Health and Institute for Molecular Medicine, and National Institute for Health and Welfare, Helsinki, Finland

MiRNAs are essential for development, function and aging of skeletal muscle, but their hormonal regulation has not been studied in muscle. We aimed to identify estrogen-regulated miRNAs and their mRNA/ protein targets in skeletal muscle of healthy, postmenopausal women.

We used monozygotic co-twin control design including nine twin pairs discordant for long-term estrogen-based Hormone Replacement Therapy (HRT). MiRNA expression profiles were determined from vastus lateralis muscle biopsies. Observed differences were confirmed by qRT-PCR. SID1.0 and DIANA microT3 were used for predicting common putative target genes and deregulated miRNA pathways. Predicted miRNA-mRNA binding and protein expression modulation, together with estrogen responsiveness, were confirmed in MCF-7 cell line. MiR-182, miR-223 and miR-142-5p expression levels were found to be significantly lower in muscles of HRT users in comparison to their non-user co-twins. MiR-182 and miR-223 target IGF-1R, FOXO1A and FOXO3A transcripts and thus putatively involve IGF-1/ insulin pathway control. Interestingly, we found significantly higher $I G F-1 R$ and FOXO3A transcript levels in HRT-users than non-users. In vitro experiments confirmed miR-182 and -223 binding with $I G F$ $1 R, F O X O 1 A$ and FOXO3A mRNAs, while estradiol treated MCF-7 cells showed dose-dependent miR-182 and -223 down-regulation concomitant with up-regulation of FOXO3A and IGF-1R mRNA and protein levels. FOXO1A showed a weaker modulation.

We identified for the first time estrogen-regulated miRNAs, such as miR-182 and miR-223, in human skeletal muscle. As they are involved in IGF-1 pathway regulation, a novel miRNA-based mechanism for estrogen to impact positively on protein balance and insulin sensitivity in postmenopausal women could be suggested.

Supported by the EU Framework 7 Project MYOAGE: Understanding and Combating age related muscle weakness Grant Agreement Number: 223576.

\section{4}

A novel approach for transcriptional fiber typing in mouse hind limb muscles

C. Bean ${ }^{1}$, P. Cancellara ${ }^{2}$, F. Chemello ${ }^{1}$, G. Busolin ${ }^{1}$, P. Laveder ${ }^{1}$, C. Reggiani ${ }^{2}$, G. Lanfranchi ${ }^{1}$

${ }^{1}$ University of Padua, Department of Biology, Padua, Italy; ${ }^{2}$ University of Padua, Department of Biomedical Sciences, Padua, Italy

Skeletal muscle fibers are heterogeneous in their metabolic, electrical, and contractile properties. The analysis of fiber type provides useful information not only about muscle function but also about its development, plasticity, and innervation. Genome-wide transcriptome analysis at the single fiber level offers a chance to profile the complete spectrum of muscle fibers. We applied this novel approach in a 
representative population of individual fibers isolated from murine EDL and soleus muscles, classified according to myosin heavy chain (MyHC) isoforms. Microarray experiments identified a thousand of fiber-specific genes. According to these signatures, fibers appear to cluster in 3 major classes, that only partially fit with the traditional MyHC classification. The analysis of the fiber-specific genes allowed the identification of the best transcriptional markers for fiber type characterization. q-PCR experiments suggest that a reliable classification is obtained with only 6 of these marker transcripts and results can be extended to fibers belonging to other hind limb muscles like tibialis anterior and gastrocnemius.

Thus, our approach offers a new tool that should facilitate analysis of samples with limiting starting material. In fact, immuno-histochemical techniques or gel electrophoresis to characterize MyHC isoforms are very time-consuming and generally restricted to sections of muscle fibers. In some instances, MyHC content can change in different region of a single fiber. Moreover, since MyHC proteins have a slow turnover, we anticipate that these transcriptional markers should be used in dynamic models of muscle plasticity to study the transition from the fast-glycolitic to the slow- oxidative phenotypes and vice versa.

195

Kinetic analysis of skeletal and cardiac human myosin isoforms M.J. Bloemink ${ }^{1}$, J.C. Deacon ${ }^{2}$, L.A. Leinwand ${ }^{2}$, M.A. Geeves ${ }^{1}$

${ }^{1}$ University of Kent, School of Biosciences, Canterbury, UK; ${ }^{2}$ University of Colorado, Cellular \& Developmental Biology, Department of Molecular, Boulder, Co, USA

Striated muscle fibres can be classified as either slow/type I or fast/type II. Within each fibre group there is a range of contractile velocities, correlating with ATPase activity and the type of myosin heavy chain isoform (MyHC) predominantly expressed. Fast skeletal fibres express one or more of the six type II skeletal muscle myosins, including the developmental MyHC (embryonic and perinatal), the adult-fast MyHC (IIa,IIb and IId), and the specialized MyHC-extraocular which all share $85-93 \%$ of their sequence. Slow/type I fibers express $\beta$ myosin which is also expressed in the heart. $\alpha$-myosin is another cardiac myosin isoform which shares $93 \%$ of its sequence with $\beta$ cardiac.

Recently a mammalian expression system for the motor domains of human muscle myosin IIs was reported and showed that the ATPase and motility properties of the adult fast skeletal myosin motors are diverse [1]. Using stopped-flow measurements we now present kinetic data for the recombinant motor domains of four skeletal myosin S1 isoforms (IIa, IIb, IId, and extraocular (exoc)) and of the two cardiac myosin $\mathrm{S} 1$ isoforms $\alpha$ and $\beta$ [2]. The most striking difference between these 6 isoforms is their affinity for ADP in the presence of actin $\left(\mathrm{K}_{\mathrm{AD}}\right)$. They also show variation in the maximum rate of ATP-induced dissociation from actin $\left(\mathrm{k}^{\prime}+2\right)$ and apparent ATP-affinity $\left(1 / \mathrm{K}_{1}{ }^{\prime}\right)$. These constants are thought to limit the shortening velocity of muscle fibres and confirm that the isoforms have distinct properties allowing each to play a unique role in human muscle physiology.

\section{References}

[1] Resnicow D.I. et.al. PNAS, (2010), 107, pp 1053-1058.

[2] Deacon J. C. et al. Cell. Mol. Life Sci, (2012), Feb 16 (online).

\section{9}

Modeling calcium diffusion, binding and uptake in a spatially realistic 3-dimentional sarcomere model

\section{R.J. Holash, B.R. MacIntosh}

Faculty of Kinesiology; University of Calgary, Calgary, AB, $\mathrm{T} 2 \mathrm{~N}-1 \mathrm{~N} 4$
The purpose of this study was to examine the release, binding and uptake of calcium ions $\left(\mathrm{Ca}^{2+}\right)$ in a spatially realistic 3-dimensional model of the sarcomere. The release of $\mathrm{Ca}^{2+}$ into a sarcomere is the event that triggers muscle contraction, and the force that a muscle produces is related to binding of $\mathrm{Ca}^{2+}$ to troponin-c. As $\mathrm{Ca}^{2+}$ is removed from the myoplasm and pumped back into the SR, contraction force falls and returns to a resting state. The details of almost every step of this process, from $\mathrm{Ca}^{2+}$ release to binding then uptake have been explored and described in tremendous detail. However, the effect of the physical structure of the cell, on diffusion, binding and uptake of $\mathrm{Ca}^{2+}$ has not been explored.

We constructed a $1 / 2$ sarcomere with realistic micro architecture using crystallographic protein reconstructions from the protein database, lattice geometry from x-ray diffraction, and triad and SR structures from electron microscopy. The sarcomere was modeled at a length of $2.2 \mu \mathrm{m}$ and at a diameter of $1.0 \mu \mathrm{m}$. The resulting mesh model was exported into Mcell3 $3^{\mathrm{TM}}$, where $\mathrm{Ca}^{2+}$ release from SR, diffusion through the filament lattice, binding to troponin-c, and subsequent uptake by calcium-ATPase, were examined. The model was evaluated at 4 locations length-wise, and within each of these, at three locations radially. Differences in diffusion, binding and subsequent uptake in each section of the model were examined and compared for a single release of calcium that approximated a single twitch. Differences in diffusion in lateral versus radial directions were noted.

200

\section{Myosin VI in myoblasts: its possible involvement in muscle} differentiation

\section{M.J. Rędowicz, K. Justyna, M. Łukasz}

Nencki Institute of Experimental Biology, Department of Biochemistry, Laboratory of Molecular Basis of Cell Motility, Warsaw, Poland

Myosin VI (MVI) is the only known myosin walking towards the minus end of actin filaments and is involved in both endocytic and exocytic membrane-trafficking, in cell spreading and migration, and possibly in gene expression. Mohiddin et al. (2004) reported that in humans a point mutation (His246Arg) within the MVI gene was associated not only with sensorineural deafness but also with mild symptoms of hypertrophic cardiomyopathy. Our previous studies on rat skeletal hindlimb muscles revealed that MVI was associated with sarcoplasmic reticulum, postsynaptic region of neuromuscular junction and muscle nuclei, and its distribution was dependent on muscle innervation.

To study whether MVI could be involved in skeletal muscle myogenesis, the widely accepted mice $\mathrm{C} 2 \mathrm{C} 12$ myoblast model was used. RT-PCR revealed no significant changes in MVI expression during myoblast differentiation stimulated by addition of horse seum. However, we observed significant differences in its cellular distribution during differentiation. In undifferentiated oval-shaped myoblasts (0-3 days) MVI was uniformly distributed within the cytoplasm but was not present in the nuclei. In 3-5-day myoblasts, which started to elongate and align MVI was also present in the distal parts of cell protrusions, and in 7-10-day myotubes it localized to myotube distal parts and was concentrated in perinuclear region, as well its presence in the nuclei was observed.

Inhibition of MVI expression by means of siRNA technique caused significant changes in myoblast morphology and proliferation, indicating its possible role in muscle differentiation.

203

\section{Remobilization through stretching improves muscle recovery} in the rat

L.O. Cação-Benedini ${ }^{1}$, P.G. Ribeiro ${ }^{1}$, A.R.S. Gomes ${ }^{2}$, J.L. Ywazaki ${ }^{2}$, A.C. Mattiello-Sverzut ${ }^{1}$ 
${ }^{1}$ University of São Paulo, Ribeirão Preto School of Medicine, Department of Biomechanics, Medicine, and Rehabilitation of the Locomotor Apparatus, Ribeirão Preto, Brazil; ${ }^{2}$ Federal University of Paraná, Department of Physiotherapy and Masters and Doctorate Programs in Physical Education, Matinhos, Brazil

Muscle performance is affected by muscle disuse. The joint restrictions and muscle shortening could regress with therapeutic protocol as muscular passive stretching. The remobilization of previously immobilized rats by free movement alone or associated with intermittent passive manual stretching (IPMS) was assessed by analysis of dorsiflexion range of motion (ROM), addition of serial sarcomeres within the fibers of the soleus muscle, intracellular morphology and expression of extracellular matrix proteins, which mechanically limit the ROM.

Sixty-four adult female Wistar rats were divided into 8 groups: immobilized (I), immobilized control $\left(\mathrm{C}_{(\mathrm{Immob})}\right)$, immobilized/stretched for 1,3 or 10 days (IS $\left.(1), \mathrm{IS}_{(3)}, \mathrm{IS}_{(10)}\right)$, immobilized/free for 1,3 or 10 days $\left(\mathrm{IF}_{(1)}, \mathrm{IF}_{(3)}, \mathrm{IF}_{(10)}\right)$. The right hind limbs were immobilized for 10 days in the plantar-flexion-shortened position. After the immobilization, the animals were submitted to IPMS. ROM, serial sarcomere numbers, expression levels of fibronectin and types I and III collagen were analyzed.

I group presented alterations in size and shape of the fibers, reduced the ROM, increased the intracellular fibronectin/total number of fibers ratio (FIF/TNFr), and the expression of the collagen types I and III. The groups $\mathrm{IS}_{(3)}$ and $\mathrm{IF}_{(3)}$ presented morphological changes exacerbated: intense cellularity, nuclear centralization, inclusion bodies, necrosis. Furthermore, ROM restriction, increased of the FIF/ TNFr and of immunoreactivity of the collagen type III been found. The abnormalities related to ROM and the morphological changes induced by immobilization were reduced in the $\mathrm{IS}_{(10)}$ group. Stretching increased the effectiveness of remobilization recovering the abnormalities observed in the muscle.

\section{5}

Partner mapping of azidoblebbistatin, the novel photo-inducible myosin inhibitor

M. Képiró, B.H. Várkuti, G. Hegyi, M. Kovács,

A. Málnási-Csizmadia

Eötvös Lóránd University, Department of Biochemistry, Budapest, Hungary

Photoreactive molecules like aryl azides play important role in life sciences, as practical tools to achieve precisely timed covalent crosslinks between ligands and targets. Using a robust technique we have synthesized azidoblebbistatin, which is a new derivative of blebbistatin, the most widely used myosin inhibitor.

In the absence of UV irradiation azidoblebbistatin and blebbistatin exhibits identical inhibitory properties. Using UV light azidoblebbistatin can be covalently crosslinked to myosin in the presence of different nucleotides, resembling different states of the myosin enzyme cycle, whereas the unbound inhibitor molecules become inactive. Interestingly, peptide analysis revealed that the binding site of azidoblebbistatin differs within the different conformational states of myosin. Applying crosslinking cycles even the low affinity partners can be covalently saturated which eliminates limitations associated with the relatively low myosin affinity and water solubility of blebbistatin. Importantly, the wavelength used for photo-crosslinking is not toxic for cells and tissues, which confers a great advantage in in vivo tests. Using Dictyostelium discoideum cell lysate we performed interactomic investigation to identify the previously unknown targets of blebbistatin utilizing the self- fluorescence of azidoblebbistatin. Since the crosslinking was performed applying increasing concentration of azidoblebbistatin, densitometry of the fluorescent bands revealed the apparent binding constant of azidoblebbistatin. With this technique the strongest interactant was found to be myosin II $\left(\mathrm{EC}_{50}=5 \mu \mathrm{M}\right)$ while eight weak partners $\left(\mathrm{EC}_{50}>30 \mu \mathrm{M}\right)$ were also detected including vacuolar $\mathrm{H}^{+}$-ATPase $\left(\mathrm{EC}_{50}=50 \pm 31 \mu \mathrm{M}\right)$, malate dehydrogenase $\left(\mathrm{EC}_{50}=55 \pm 17 \mu \mathrm{M}\right)$ and elongation factor $1 \alpha\left(\mathrm{EC}_{50}>100 \mu \mathrm{M}\right)$.

\section{6}

\section{An actomyosin model of the initial state of the powerstroke}

\author{
B.H. Várkuti, Z. Yang, A.Á. Rauscher, M. Képiró,
} A. Málnási-Csizmadia

Eötvös Loránd University, Department of Biochemistry, Budapest, Hungary

The mechanism of myosin's actin activation is not known, mainly due to the absence of actomyosin crystal structures. Here we present a novel in silico model of actomyosin at the start of the powerstroke, representing the major changes that are induced by actin binding to myosin, and experimental evidence of these changes. An actin trimer was docked and relaxed with myosin in its weak actin binding, up lever state (open actin-binding cleft and closed switch II loop). Interestingly, in the nanosecond timescale actin binding induced a further up movement of the lever accompanied with a further closure of the switch II loop. This state may represent a'cocked' state of myosin that precedes the powerstroke. Additionally, when the interaction between activation loop and actin $\mathrm{N}$-terminus was interrupted in the docked and relaxed actomyosin by applying a mutation, myosin relaxed back to its actin detached up lever conformation.

In order to test this in silico finding we experimentally examined the lever movement upon actin binding. GFP and a biarsenical fluorophore were introduced into the C- and N-terminal of Dictyostelium myosin motor domain in order to follow the lever position by FRET. We conclude that in silico and experimental results reveal a new state of actomyosin at the beginning of the powerstroke, indicating the initial mechanism of actin activation of myosin.

\section{7}

Passive stretching increases sarcolemmal injury and reduces collagen Type $I$ in rat soleus muscle immediately after immobilization

\section{L.O. Cação-Benedini ${ }^{1}$, P.G. Ribeiro ${ }^{1}$, C.M. Prado $^{2}$,} A.C. Mattiello-Sverzut ${ }^{1}$

University of São Paulo, Ribeirão Preto School of Medicine, Department of Biomechanics, Medicine, and Rehabilitation of the Locomotor Apparatus, Ribeirão Preto, Brazil; ${ }^{2}$ University of São Paulo, Department of Pathology, Ribeirão Preto School of Medicine, Ribeirão Preto, Brazil

Costamere proteins and extracellular matrix compounds are susceptible to injury during the early phase of exercise. Using longitudinal sections of the soleus skeletal muscle, this study investigated the changes induced by passive stretch training with the free movement after immobilization of the hind limb of rats.

Sixty-four adult female Wistar rats were divided into 8 groups: immobilized (I), immobilized control $\left(\mathrm{C}_{(\mathrm{Immob})}\right)$, immobilized/stretched for 1,3 or 10 days $\left(\mathrm{IS}_{(1)}, \mathrm{IS}_{(3)}, \mathrm{IS}_{(10)}\right)$, and immobilized/free for 1,3 or 10 days $\left(\mathrm{IF}_{(1)}, \mathrm{IF}_{(3)}, \mathrm{IF}_{(10)}\right)$. The right hind limbs were immobilized for 10 days in the plantar-flexed (short) position. Immediately after removal of the device, the animals were submitted to passive stretch. The soleus muscle was processed for immunofluorescence (laminin, dystrophin, macrophages) and Western blotting (dystrophin, laminin, types I and III collagen).

Hypokinesia increased the expression of dystrophin and laminin, but reduced the expression of type I collagen. $\mathrm{IS}_{(3)}$ increased the 
expression of dystrophin and led to an increase in the number of macrophages. During remobilization by both programs (stretched and free movement), analysis of the ratio of type I to type III collagen showed a higher amount of type III collagen.

The reduced expression of collagen type I after immobilization suggests increased susceptibility of the muscle fibers to injury. Remobilization using longitudinal stress combined or not with passive stretching increased sarcolemmal injury immediately after removal of the device.

\section{8}

Heart specific interference of Surf1 and Scox in Drosophila Melanogaster cause dilated cardiomyopathy

L. Martinez-Morentin ${ }^{1}$, B. Molina ${ }^{1}$, K. Ocoor ${ }^{2}$, R. Bodmer ${ }^{2}$, M. Cervera ${ }^{1}$, J.J. Arredondo ${ }^{1}$

${ }^{1}$ Universidad Autonoma de Madrid, Facultad de Medicina, Ins. Inv. Biomedicas-Dpt. de Bioquimica, Madrid, Spain; ${ }^{2}$ Sanford Burnham Medical Research Institute, San Diego USA

Mitochondria are organelles of vital importance in eukaryotes. Its main function is the production of energy by a process called oxidative phosphorylation. Mitochondrial disorders most often cause a defective OXPHOS, being Complex IV malfunction, due to mutations in assembly factors Sco2 or Surf1, one of the most common.

In the recent years, Drosophila has emerged as a powerful model system for heart human disease, which, in some cases, has allowed the identification of new genes involved in human cardiomyopathies. Using Drosophila melanogaster as model system, we have set up the heart specific interference of ScoX and Surf1 to examine their in vivo role in cardiac physiology. Hearts from interfered flies display a reduced fractional shortening, due to a bigger systolic and diastolic diameter, an increased incidence of arrhythmias, and prolonged diastolic and systolic intervals. Heart structural analysis showed a strong increase in conical chamber size (Fig. 1) in interfered flies. We also observed evident abnormalities in the cellular architecture of cardiomyocytes, as severely misaligned myofibrils, or the displacement of ostia cells.

We conclude that heart specific interference of Surf1 and ScoX compromises heart function reminiscent of Dilated Cardiomyopathy as evidenced by the observed reduction in heart rate and cardiac contractility together with an increase in the arrhythmia index and an increase in conical chamber size.
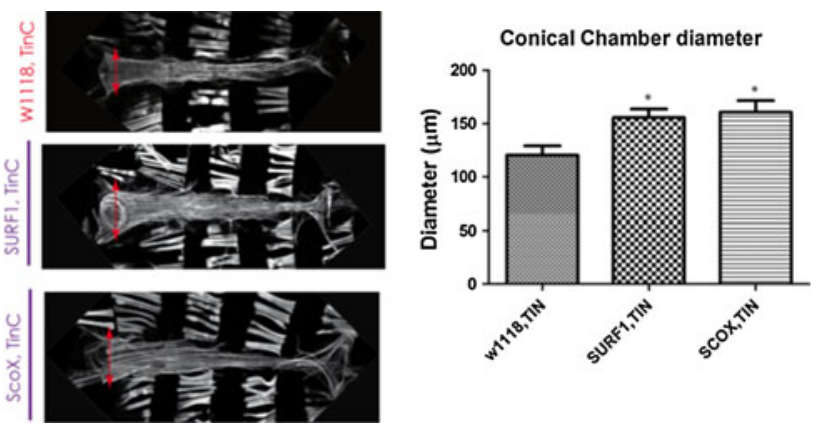

Fig. 1 ScoX and Surf1 interfered flies display a larger conical chamber diameter. Phalloidin stained hearts (left) and Conical Chamber diameter (right, $* P<0,01$ ) from control and ScoX or Surf1 interfered animals

\section{9}

Atomic-level visualization of smooth muscle activation by myosin RLC phosphorylation

D.D. Thomas, B.A. Colson, M.A. Mauseth, D.J. Kast

University of Minnesota, Dept. of Biochemistry, Molecular Biology and Biophysics, Minneapolis, MN, USA

We have engineered site-directed probe pairs in the regulatory light chain (RLC) for exchange into smooth muscle heavy meromyosin (HMM) and subfragment-1 (S1), in order to examine the phosphorylation-induced changes in RLC structural states using time-resolved FRET (TR-FRET) and EPR. Phosphorylation of the RLC is required for activation of contraction in smooth muscle and modulates force in striated muscle. The auto-inhibited state of smooth muscle myosin involves interactions between its two catalytic domains, and force development is triggered when this inhibitory conformation of two-headed myosin is alleviated by phosphorylation of the RLC's $\mathrm{N}$-terminal phosphorylation domain (PD) at S19. Since crystal structures of the RLC include its core lobes, but not the PD, the mechanism by which RLC phosphorylation allosterically triggers disruption of HMM auto-inhibition to regulate contraction remained unresolved.

Spectroscopic studies of smooth muscle regulation using sitedirected spin and fluorescent probes resolved intramolecular atomic distance measurements between the RLC's core lobes and PD, revealing both ordered and disordered structural states, and MD simulations extended these observations to yield an atomic-resolution structural trajectory of the phosphorylation-dependent activation mechanism.

Based on intramolecular FRET, we found that smooth muscle RLC always assumes two structural states in both biochemical states of activation: phosphorylated RLC shifts the equilibrium of these two structural states from favoring the compact closed state to the extended open state of the PD, relative to the RLC core. Both states are resolved in both single-headed S1 and double-headed HMM, but the open state in unphosphorylated HMM is more compact, presumably due to headhead interactions. All-atom MD simulations, carried out for $70 \mathrm{~ns}$ each, corroborate the molar distributions and atomic distances of our TR-FRET studies. Furthermore, MD simulations atomic-resolution insight into the mechanism of smooth muscle activation, revealing a specific interdomain (PD and C-lobe) salt-bridge that stabilizes the closed state and a specific intradomain salt-bridge in the PD (involving phosphoserine) that stabilizes the open state. These hypotheses are being tested experimentally by site-directed mutagenesis to manipulate charge, followed by labeling, TR-FRET, and EPR.

By combining site-directed labeling, FRET, EPR, and MD simulations, we have found that the N-terminal phosphorylation domain of smooth muscle myosin RLC undergoes a disorder-to-order transition upon phosphorylation, mediated by changes in interdomain and intradomain salt bridges, and by changes in head-head interactions. This study provides the first atomic-resolution insight into the structural dynamics of RLC phosphorylation, and a similar approach should be applicable to striated muscle.

\section{0}

Conformation of the troponin core domain during relaxation and active contraction of striated muscle

\section{A.C. Knowles, M. Irving, Y.B. Sun}

Randall Division of Cell and Molecular Biophysics and British Heart Foundation Centre of Research Excellence, King's College London, London SE1 1UL, UK

Contraction of striated muscles is regulated by $\mathrm{Ca}^{2+}$ ions binding to troponin in the actin-containing thin filament, leading to a movement of 
tropomyosin around the filament that allows myosin heads to bind to actin and generate force. However the molecular structural basis of this troponin-mediated signalling pathway has so far remained obscure.

Here we use polarized fluorescence from bifunctional rhodamine to determine the orientation of the major component of troponin core domain- the 'IT arm'- on the thin filaments of skeletal muscle. We show that the coiled-coil between the troponin I and $\mathrm{T}$ chains that forms the backbone of the IT arm makes an angle of $\sim 55^{\circ}$ with the filament axis in relaxed muscle. This angle decreases slightly on activation, but this is a secondary effect of myosin heads binding to actin rather than an essential step in the $\mathrm{Ca}^{2+}$ signalling pathway. By combining the new in situ orientation data with published in vitro measurements of intermolecular distances, we derive a model for the in situ structure of the thin filament. In this model the IT arm holds the $\mathrm{N}$-terminal lobe of troponin $\mathrm{C}(\mathrm{NTnC})$ close to the actin filament surface, with the binding site for the TnI switch peptide facing away from the filament surface.

This conformation suggests a regulatory mechanism in which $\mathrm{Ca}^{2+}$-dependent binding of the switch peptide to NTnC disrupts the binding of the flanking TnI inhibitory regions to actin and tropomyosin, in turn allowing tropomyosin to move azimuthally to uncover the myosin binding sites on actin.

\section{1}

A novel mouse model of nebulin-based nemaline myopathy

C. Ottenheijm ${ }^{1,2}$, D. Buck ${ }^{2}$, J. de Winter ${ }^{1}$, M. Lawlor ${ }^{3}$, G. Stienen ${ }^{1}$, A. Beggs ${ }^{3}$, S. Labeit ${ }^{4}$, H. Granzier ${ }^{2}$

${ }^{1}$ VU University Medical Center, Department of Physiology, Amsterdam, the Netherlands; ${ }^{2}$ University of Arizona, Department of Physiology, Tucson, AZ, USA; ${ }^{3}$ Harvard Medical School, Division of Genetics and Program in Genomics, The Manton Center for Orphan Disease Research, Children's Hospital Boston, Boston, MA, USA;

${ }^{4}$ University of Heidelberg, Department for Integrative

Pathophysiology, Medical Faculty Mannheim, Germany

Nebulin-a giant sarcomeric protein-plays a pivotal role in skeletal muscle contractility by regulating thin filament length and function. Although mutations in the gene encoding nebulin $(N E B)$ are a frequent cause of nemaline myopathy (NM), the most common nondystrophic congenital myopathy, the mechanisms by which mutations in $N E B$ cause muscle weakness remain largely unknown. To better understand these mechanisms, we have generated a mouse model in which $N E B$ exon 55 is deleted (NEB $\triangle$ ex55), a mutation known to frequently occur in NM patients.

NEB $\Delta$ ex 55 mice are born close to Mendelian ratio's, but show growth retardation after birth. Electronmicroscopy shows nemaline rods-a hallmark feature of NM-in muscle fibers from NEB $\Delta$ ex 55 mice. Western blotting studies with nebulin-specific antibodies reveal much reduced nebulin levels in muscle from NEB $\Delta$ ex55 mice. Immunofluorescence confocal microscopy studies with tropomodulin antibodies and phalloidin reveal that thin filament length is reduced in muscle fibers from NEB $\Delta$ ex 55 mice. In line with reduced thin filament length, the maximal force generating capacity of skinned muscle fibers is reduced in NEB $\Delta$ ex 55 mice with a more pronounced reduction at longer sarcomere lengths. Finally, in NEB $\Delta$ ex 55 mice the regulation of contraction is impaired, as evidenced by marked changes in cross bridge cycling kinetics and by a reduction of the calcium sensitivity of force generation. This reduced calcium sensitivity was observed only at short sarcomere lengths, suggesting that nebulin might play a role in the length dependence of activation.

In conclusion, we have generated the first nebulin-based NM model. Our data indicate that the phenotype of NEB $\Delta$ ex55 mice closely recapitulates that observed previously by us in patients harboring this particular mutation.

\section{4}

MURF2 targets serum response factor (SRF) for ubiquitin-mediated degradation

\section{S. Perera, M. Gautel}

King's College London, Randall Division of Cell and Molecular Biophysics, Cardiovascular Division and BHF Centre of Research Excellence, London, UK

MURF2 belongs to the muscle-specific ring finger family of E3 ubiquitin ligases, which contain the RING-finger, B-box and coiledcoil tripartite motif (Spencer et al. 2000; Centner et al. 2001). The three MURF genes identified to date (MURFs 1, 2 and 3) encode highly homologous proteins (Centner et al. 2001). MURF1 null mice show resistance to steroid- and disuse-induced muscle atrophy (Bodine et al. 2001). Both MURFs 1 and 3 participate in the degradation of sarcomeric proteins (Fielitz et al. 2007).

We recently showed that MURF2 is the first MURF isogene to be embryonically expressed in both cardiac and skeletal muscle whilst MURFs 1 and 3 are upregulated postnatally (Perera et al. 2011, Perera et al. 2012). siRNA experiments confirmed that MURF2 plays a vital role in myofibrillar assembly and turnover (Perera et al. 2011). However, the E3 ubiquitin ligase activity of MURF2 still remained obscure. SRF - a known target of MURF2 (Lange et al. 2005) is also expressed embryonically but is rapidly downregulated in parallel with increasing levels of MURF2 (Perera et al. 2011, Perera et al. 2012). Given the established E3 ligase activity for MURFs 1 and 3, we predicted that MURF2 may also function in a similar capacity. Our data show for the first time that MURF2 cooperates with the E2 ubiquitin-conjugating enzymes UbcH5a, b and c to ubiquitinate SRF in vitro and in vivo, targeting it for degradation. These findings uniquely implicate MURF2 in the regulation of nuclear transcription factors and associated downstream signalling pathways.

\section{5}

Fatigue effects on ATP-induced actomyosin dissociation kinetics at $35^{\circ} \mathrm{C}$

\section{Karatzaferi ${ }^{1}$, M.A. Geeves ${ }^{2}$}

${ }^{1}$ University of Thessaly, DPESS, Muscle Physiology \& Mechanics Group, Trikala, GR; ${ }^{2}$ University of Kent, Biosciences, Molecular Motors \& the Cytoskeleton Group, Canterbury, UK

The functional characteristics of the various types of skeletal muscles are largely dictated by the expression of class II myosin heavy chains (MyHC) isoforms. For example, higher levels of a fast MyHC isoform lead to faster contraction velocity, while more slow MyHC lead to a slower velocity.

In slow myosin isoforms ADP binding to A.M is tighter than with fast isoforms. Most kinetics characterisations in the past have used 'standard' conditions e.g. $20^{\circ} \mathrm{C}, \mathrm{pH} 7$ and no added phosphate. However, in vivo muscle temperature ranges from 34 to $>40{ }^{\circ} \mathrm{C}$ while in extreme fatigue, $\mathrm{pH}$ drops and inorganic phosphate (Pi) accumulates. Recent data by $\mathrm{CK}$ highlighted the importance of possible synergisms between temperature and such 'fatigue' factors; effects which may differ in the different fibre types.

We examined the fast kinetics of ATP induced dissociation of A.M. ( \pm ADP) of rabbit fast and slow myosin for a range of temperatures using stopped flow; experiments were repeated in 'fatigue' conditions (pH6.2, $30 \mathrm{mM} \mathrm{Pi}$, singly or in combination).Neither temperature nor $\mathrm{pH}$ had an evident effect on ADP affinity for the fast myosin. For slow myosins, ADP affinity weakened at $\mathrm{pH}$ 6.2, at both $20 \& 35^{\circ} \mathrm{C}$. Addition of Pi affected the two myosins differently, Pi competing with the ADP inhibition in fast but almost abolishing ADP inhibition in slow myosins. 
Our results provide further evidence of distinct mechanochemical coupling between the myosin types.

Supported in part by the European Union Social Fund, Operational Programme Education and Lifelong learning, NSRF 2007-2013 Programme for Development, Project Thalis Muscle-Fun (MIS: 377260).

\section{8}

\section{Insulin as a modulator of cardiac titin}

A.E. Müller ${ }^{1,3}$, K. Babicz ${ }^{3}$, S. Bongardt ${ }^{1}$, W. Röll ${ }^{2}$, W.A. Linke ${ }^{3}$, M. Krüger ${ }^{1,3}$

${ }^{1}$ Heinrich Heine University Düsseldorf,Dept. of Cardiovascular Physiology, D-40225 Düsseldorf, Germany; ${ }^{2}$ University of Bonn, Dept. of Cardiac Surgery, Germany; ${ }^{3}$ Ruhr University Bochum, Dept. of Cardiovascular Physiology, D-44801 Bochum, Germany

The passive mechanical properties of cardiac titin depend on the expression ratio of the isoforms $\mathrm{N} 2 \mathrm{BA}$ and $\mathrm{N} 2 \mathrm{~B}$, and can be dynamically modified by phosphorylation through PKA, PKG and PKC.

Here we studied the influence of insulin on titin isoform composition and phosphorylation using cultured cardiomyocytes and diabetic human heart tissue. Titin isoform composition and phosphorylation was analyzed using $1.8 \%$ SDS PAGE stained with either Coomassie, or the phosphoprotein stain ProQ-Diamond in combination with SYPRO-Ruby.

In embryonic rat cardiomyocytes (CMs) insulin treatment for 7 days significantly increased the relative expression of stiff N2B-titin by $>12 \%$ compared to untreated cells. Moreover, in embryonic and adult rat CMs insulin caused a major increase in the relative titin phosphorylation within 15 min of treatment. This effect was blocked by inhibition of the NO/cGMP- pathway. We further investigated the influence of altered insulin homeostasis on titin isoform ratio and titin phosphorylation using samples from right atria of diabetic and non diabetic patients that underwent cardiac surgery due to coronary artery disease. Diabetic heart samples showed a major increase in titin N2BA expression that was most pronounced in patients with a high HbA1c $(>7.0 \%)$. First analyses also demonstrate a hypophosphorylation of total titin from diabetic hearts, probably caused by reduced phosphorylation of the PEVK element of titin, as indicated by Western blot analyses using phospho-specific antibodies.

We conclude that insulin is a strong modulator of cardiac titin, and may therefore play an important role in modifying myocardial stiffness in human hearts.

\section{0}

\section{Cardiac myosin binding protein-C restricts intrafilament torsional dynamics of actin in a phosphorylation- dependent manner}

B.A. Colson ${ }^{1}$, I.N. Rybakova ${ }^{2}$, E. Prochniewicz ${ }^{1}$, R.L. Moss ${ }^{2}$, D.D. Thomas

${ }^{1}$ University of Minnesota, Dept. of Biochemistry, Molecular Biology and Biophysics, Minneapolis, MN, USA; ${ }^{2}$ University of Wisconsin School of Medicine and Public Health, Department of Cell and Regenerative Biology, Madison, WI, USA

We have determined the effects of myosin binding protein-C (MyBPC) and its domains on the microsecond time-scale rotational dynamics of actin, using time-resolved phosphorescence anisotropy (TPA). MyBP-C is a multi-domain thick filament-associated modulator of striated muscle contraction, interacting with myosin, titin, and possibly actin. Cardiac and slow skeletal MyBP-C are known substrates for Protein kinase-A (PKA), and phosphorylation of the cardiac isoform alters contractile properties and myofilament structure.

To determine the effects of MyBP-C on actin's microsecond structural dynamics, we labeled actin at C374 with erythrosine iodoacetamide and performed TPA experiments.

The interaction of all three MyBP-C isoforms with actin increased the final anisotropy $(\mathrm{r} \infty)$ of the TPA decay in a concentrationdependent manner, indicating restriction of the amplitude of actin torsional flexibility by $15-20^{\circ}$ at saturation of the TPA effect. PKA phosphorylation of slow skeletal and cardiac MyBP-C relieved the restrictive effect on actin torsional flexibility, and at the same time decreased the rate of intrafilament motion. In the case of fast skeletal MyBP-C, its effect on actin dynamics was unchanged by phosphorylation. Effects of truncated cardiac MyBP-C on actin anisotropy revealed that the $\mathrm{C}$-terminal half $\mathrm{C} 5-\mathrm{C} 10$ has partial effects of fulllength cMyBP-C on amplitude and rate, and binds actin more tightly than the N-terminal half $\mathrm{C} 0-\mathrm{C} 4$, which does not affect amplitude but increases the rate of actin intrafilament motion.

These MyBP-C-induced changes in actin dynamics may play a role in the known effects of MyBP-C on the functional actin-myosin interaction.

Colson is a candidate for a young investigator award to be judged during the EMC 2012.

\section{1}

\section{Impairment of arginyltransferase enzyme (ATE1) in the heart} reduces myofibril contractile forces in adult mice

A.S. Cornachione ${ }^{1}$, A. Kalganov ${ }^{1}$, P.A. Ribeiro ${ }^{3}$, F. Minozzo ${ }^{1}$, N.A. Leu ${ }^{2}$, S. Kurosaka ${ }^{2}$; A. Kashina ${ }^{2}$, D.E. Rassier ${ }^{1}$

${ }^{1}$ Department of Kinesiology and Physical Education, Physics and Physiology, McGill University, Montreal, Canada; ${ }^{2}$ Department of Animal Biology School of Veterinary Medicine, University of Pennsylvania, USA; ${ }^{3}$ Universidade Federal do Rio grande do Sul, Porto Alegre, Brazil

The enzyme arginyltransferase (ATE1) is responsible for transferring arginine (Arg) from arginyl-tRNA onto different proteins. This arginylation process is essential for heart formation and angiogenesis. Impairment in ATE1 can cause defects in heart development and myofibril disorganization. In this study, we compared contractile properties of single myofibrils isolated from the cardiac muscle of the young and adult knockout (Ate1 KO). Small bundles of myofibril isolated from ventricles in mice with different ages (range: 5-12 months) were attached to atomic force cantilevers. Active force, passive force and the rates of force development $\left(\mathrm{K}_{\mathrm{act}}\right)$, redevelopment $\left(\mathrm{K}_{\mathrm{tr}}\right)$ and relaxation $\left(\mathrm{K}_{\mathrm{rel}}\right)$ were measured in sarcomeres lengths (SL) ranging between 1.8 and $2.6 \mu \mathrm{m}$. Myofibrils from older Ate1 $\mathrm{KO}$ mice showed lower isometric forces $(102.2 \pm 11.0 \mathrm{nN} / \mu \mathrm{m} 2)$ than controls $(151.3 \pm 11.7 \mathrm{nN} / \mu \mathrm{m} 2)$, while no significant differences were observed in young mice. The passive forces were decreased in older $\mathrm{KO}$ mice in the range of SL investigated. $\mathrm{K}_{\mathrm{act}}$ was similar between groups (Ate1 KO: $3.1 \pm 0.4 \mathrm{~s}^{-1}$, control: $3.3 \pm 0.5 \mathrm{~s}^{-1}$ ) of older mice. $\mathrm{K}_{\mathrm{tr}}$ was also similar between groups (Ate1 KO: $4.7 \pm 2.2 \mathrm{~s}^{-1}$, control: $6.6 \pm 0.8 \mathrm{~s}^{-1}$ ).

The results of our experiments imply a close relation between arginylation and cardiac muscle contractile properties, which advances with age. Since $\mathrm{K}_{\mathrm{act}}$ and $\mathrm{K}_{\mathrm{tr}}$ did not change in $\mathrm{KO}$ mice, force may be impaired by mechanisms other than kinetics of cross-bridges transitions from weakly-bound to strongly-bound states.

Cornachione is a candidate for a young investigator award to be judged during the EMC 2012. 


\section{2}

Effect of myosin regulatory light chain (RLC) phosphorylation on the contractile properties of permeabilised cardiac trabeculae from the rat

C. Toepfer ${ }^{1}$, V. Caorsi ${ }^{2}$, T. West ${ }^{1}$, C. Mansfield ${ }^{1}$, M.C. Leung ${ }^{1}$, S. Marston ${ }^{1}$, M.A. Ferenczi ${ }^{1}$

${ }^{1}$ Imperial College London, NHLI, UK; ${ }^{2}$ Royal Veterinary College London, Structure \& Motion Laboratory, North Mymms, UK

We explore the role of RLC phosphorylation in modulating the physiological performance of cardiac muscle. Mutations in myosin RLC sometimes cause hypertrophic cardiomyopathies (HCM's), whilst altered RLC phosphorylation is implicated in progression of heart failure.

Cardiac performance was determined by measuring the Force velocity $(\mathrm{FV})$ relationship from which the power velocity $(\mathrm{PV})$ relationship was calculated. Permeabilized left ventricular trabeculae from rat hearts underwent an exchange procedure to replace endogenous RLC with differentially phosphorylated recombinant RLC. Trabeculae were activated at $20^{\circ} \mathrm{C}$ by T-jump in $32 \mu \mathrm{mol} / \mathrm{L}$ calcium. Increasing phosphorylation of the RLC increased peak force of contraction by a factor of 2.5 in comparison with de-phosphorylated RLC exchange. Notably maximal unloaded shortening velocity $\left(\mathrm{V}_{\max }\right)$ increased to $\sim 130 \%$ and peak power increased five-fold. Increasing RLC phosphorylation above native levels as observed in un-exchanged trabeculae showed a gain in function where power was increased two-fold. Decreasing RLC phosphorylation showed a two-fold decrease in power and $V_{\max }$ is reduced two-fold.

We conclude that RLC phosphorylation has a modulatory impact on cardiac muscle contraction independently of calcium sensitivity, and a role for RLC phosphorylation in the progression of many types of heart failures not directly linked to RLC mutation may be important.

\section{6}

Axial distribution of myosin binding protein-C is unaffected by mutations in human cardiac and skeletal muscle

\section{A. Vydyanath ${ }^{1}$, C.A. Gurnett ${ }^{2}$, S. Marston ${ }^{3}$, P.K. Luther ${ }^{1}$}

${ }^{1}$ Imperial College London, National Heart and Lung Institute, Molecular Medicine Section, London SW7 2AZ, UK; ${ }^{2}$ Washington University School of Medicine, Division Paediatric Neurology, Department of Neurology, St Louis, MO 63110, USA; ${ }^{3}$ Imperial College London, National Heart and Lung Institute, Myocardial Function, London SW7 2AZ, UK

Myosin binding protein-C (MyBP-C), a major thick filament associated sarcomeric protein, plays an important functional and structural role in regulating sarcomere assembly and crossbridge formation. Missing or aberrant MyBP-C proteins (both cardiac and skeletal) have been shown to cause both cardiac and skeletal myopathies, thereby emphasising its importance for the normal functioning of the sarcomere. Mutations in cardiac MyBP-C are a major cause of hypertrophic cardiomyopathy (HCM), while mutations in skeletal MyBP-C have been implicated in a disease of skeletal muscle - distal arthrogryposis type 1 (DA-1). Here we report the first detailed electron microscopy studies on human cardiac and skeletal tissues carrying MyBP-C gene mutations, using samples obtained from $\mathrm{HCM}$ and DA-1 patients. We have used established image averaging methods to identify and study the axial distribution of MyBP-C on the thick filament by averaging profile plots of the A-band of the sarcomere from electron micrographs of human cardiac and skeletal myopathy specimens. Due to the difficulty of obtaining normal human tissue, we compared the distribution to the A-band structure in normal frog skeletal, rat cardiac muscle and in cardiac muscle of MyBP-Cdeficient mice. Very similar overall profile averages were obtained from the C-zones in cardiac HCM samples and skeletal DA-1 samples with MyBP-C gene mutations, suggesting that mutations in MyBP-C do not alter its mean axial distribution along the thick filament.

This research was funded by the EC Grant Agreement n. 241577 (BIG-Heart) FP7 Seventh Framework Programme Health and the British Heart Foundation (RG/11/21/29335).

\section{7}

Understanding interaction of alpha-actinin and actin in nemaline myopathy Z-bands

\author{
E.P. Morris ${ }^{1}$, P.K. Luther ${ }^{2}$, J.M. Squire ${ }^{3}$
}

${ }^{1}$ Institute of Cancer Research, Chester Beatty Laboratories, London SW3 6JB, UK; ${ }^{2}$ Imperial College London, National Heart and Lung Institute, Molecular Medicine Section, London SW7 2AZ, UK;

${ }^{3}$ University of Bristol, Department of Physiology and Pharmacology, Muscle Contraction Group, Bristol, BS8 1TD, UK

Actin and $\alpha$-actinin are ubiquitous proteins in all cells of the animal kingdom and their interaction is of great importance in cellular architecture and cell motility. Natural crystalline arrays of the two proteins occur in nemaline rods in patients with nemaline myopathy, a disease characterised by abnormal enlargement of the Z-disc and isolated enlarged Z-discs. It is caused by mutations in thin filament proteins, mainly actin and nebulin. We have examined the fine structure in nemaline rods by electron microscopy of negatively stained cryosections. Such cryosections are renowned for delivering high resolution structural detail compared with conventionally prepared plastic sections. Using such images we have calculated the three-dimensional structure of the repeating unit of the nemaline Z-disc. This reveals two sets of oppositely oriented actin filaments connected by a series of links. Within the filaments actin subunit detail allows the accurate docking of an atomic F-actin model. Links between actin filaments can be modelled with $\alpha$-actinin thereby explaining the way in which the z-disc is held together and characterising the geometry of interaction between $\alpha$-actinin and F-actin in this system.

This research was funded by the British Heart Foundation (RG/11/ 21/29335).

228

Muscle structural and functional changes with ageing, disuse and exercise

M.V. Narici ${ }^{1,2}$, E.L. Campbell ${ }^{2}$, J. McPhee ${ }^{2}$, G. Trisolino ${ }^{3}$, O.R. Seynnes ${ }^{2}$, M. Conte ${ }^{4}$, C. Lanzarini ${ }^{4}$, L. Bucci ${ }^{4}$, M. Capri ${ }^{4}$, S. Salvioli ${ }^{4}$, C. Franceschi ${ }^{4}$, D.A. Jones ${ }^{2}$

${ }^{1}$ University of Nottingham, School of Graduate Entry to Medicine and Health, Derby, UK; ${ }^{2}$ Manchester Metropolitan University, Institute for Biomedical Research into Human Movement, Manchester, UK; ${ }^{3}$ Rizzoli Orthopedic Institute, Department of Reconstructive Hip \& Knee Joint Surgery, Bologna, Italy; ${ }^{4}$ Alma Mater Studiorum, Department of Experimental Pathology, Bologna, Italy

Sarcopenia affects $50 \%$ of males and $68 \%$ of females aged 70-79 years. This age-related loss of muscle mass not only involves atrophy of muscle fibres but also marked remodelling of muscle structure. The aims of this investigation were thus to, 1) evaluate the changes in muscle structure (architecture) and function associated with sarcopenia, and, 2) assess whether muscle atrophy caused by 
inactivity could be ameliorated by amino acid supplementation and by exercise post-inactivity.

In the MYOAGE study we investigated: 1) the architectural features (fascicle length, Lf, and muscle thickness, Tm) measured by ultrasound (in $\mathrm{m}$. vastus lateralis), and maximum isometric voluntary contraction (MVC) force of the knee extensor muscles (KE) of 24 young adults (YA) (18-35 years) and 27 active older (AO) (67-82 years), 32 frail inactive older (FO) individuals (aged 65-94 years), and 24 track and field master athletes (MA) aged 65-96 years, and 2) the architecture and MVC of the KE in 8 young men (YM) aged 18-35 years during 3 week-unilateral lower limb unloading (ULLS) followed by 3 week resistive training with either Leucine or Alanine supplementation.

1) Compared to the YA, KE force was $72 \%$ lower in FO, $34 \%$ lower in AO and $22 \%$ lower in MA. Differences were smaller but still persisted after normalisation of force to Tm, for $\mathrm{F} / \mathrm{Tm}$ was $391.0 \mathrm{~N} / \mathrm{cm}$ in YA, $315.0 \mathrm{~N} / \mathrm{cm}$ in MA, $302.5 \mathrm{~N} / \mathrm{cm}$ in $\mathrm{AO}, 93.9 \mathrm{~N} / \mathrm{cm}$ in $\mathrm{FO}$, indicating a marked deterioration in muscle quality in the $\mathrm{FO}$ and $\mathrm{AO}$, but less in MA. Tm and Lf were lower in the older individuals, particularly so in the FO. However, with increasing age and frailty Tm decreased more than Lf, with a consequential increase in the $\mathrm{Lf} / \mathrm{Tm}$ ratio. This phenomenon was absent in MA, providing additional evidence that the $\mathrm{LF} / \mathrm{t}$ ratio is a useful marker of sarcopenia. Lf/t significantly correlated with KE force and appendicular muscle mass evaluated with DXA. 2) In response to the 3 wk ULLS in YM, KE volume decreased by $10 \%$, muscle force by $26 \%$, Lf and Tm both by $9 \%$. After 3-week active recovery, all values returned to baseline levels and no effects were found with Leucine supplementation.

Older skeletal muscle is intrinsically weaker than young muscle. Inactivity drastically enhances this deterioration in muscle quality, whereas life-long physical activity by master athletes protects against the loss of muscle mass, structure and function, possibly through preservation of motor units (Power et al. 2010). Sarcopenia, involves not only a loss of muscle mass but also marked remodelling of muscle architecture; the age-related loss of geometric proportionality represents a useful 'signature of sarcopenia'. With inactivity muscle seems to become anabolically blunt as Leucine supplementation failed to mitigate atrophy with disuse or improve gains in muscle mass with exercise.

\section{References}

Power, G. A., et al. Motor Unit Number Estimates in Masters Runners: Use It or Lose It? Med. Sci. Sports Exerc., Vol. 42, No. 9, pp. 1644-1650, 2010.

Supported by the EU Framework 7 Project MYOAGE: Understanding and Combating age related muscle weakness Grant Agreement Number: 223576.

\section{9}

Characterisation of the actomyosin complex using FRET: an inside look into the molecular mechanisms of contraction in cardiac muscle

L. Chen ${ }^{1}$, V. Caorsi ${ }^{1}$, C. Toepfer ${ }^{1}$, W. Song ${ }^{2}$, S.B. Marston ${ }^{2}$, M.A. Ferenczi ${ }^{1}$

${ }^{1}$ Imperial College London, Molecular Medicine NHLI, London, UK; ${ }^{2}$ Myocardial Function, NHLI, London, UK

Elucidating actomyosin interaction is key to the understanding of molecular mechanisms of force generation in muscle. Although the swinging lever arm hypothesis is widely accepted, the precise myosin-actin interactions at different stages of the power stroke are still unclear and further studies of actomyosin complexes within functional muscle systems are required [1].
Here, the use of Förster resonance energy transfer (FRET) to measure nanometre distances between myosin and actin in functional cardiac muscle is described. In particular the interaction between the essential light chain (ELC)-AlexaFluor488 (labelled at a single cysteine in position 180 of a modified ELC, exchanged with the native one [2]) and Actin-AlexaFluor594 Phalloidin is evaluated by the acceptor photobleaching method. In addition, we exploit FRET methods to measure the effect of cardiac disease mutations, such as E99 $\mathrm{K}$, an actin mutation in a transgenic mouse model which in humans leads to hypertrophic cardiomyopathy [3].

Our preliminary data suggest: i) E99 $\mathrm{K}$ actin-mutation does not seem to affect the acto-myosin structures in terms of FRET efficiency evaluated; ii) in contrast to skeletal fibres, [4] the ELC-Actin distance in rigor cardiac fibres is within the range for FRET, indicating that cardiac and skeletal muscle may possess differing cross- bridge conformations; iii) surprisingly, the ELC-Actin distance in relaxed cardiac fibres is approximately $2 \mathrm{~nm}$ shorter than rigor-state distances. We successfully demonstrated that FRET has enough precision to detect nanometre-scale variations in the actomyosin structure in different muscle environments and that FRET may be a useful tool to determine the structural effects of disease-causing mutations at a molecular level.

\section{References}

[1] M.A. Geeves and K.C. Holmes. Adv Protein Chem. 2005; 71:161-93.

[2] J.Borejdo, D.S. Ushakov, R. Moreland, I. Akopova, Y. Reshetnyak, L.D. Saraswat, K. Kamm and S.Lowey. Biochemistry. 2001; 3:40(13):3796-803.

[3] Song W, Dyer E, Stuckey DJ, Copeland O, Leung MC, Bayliss C, Messer A, Wilkinson R, Tremoleda JL, Schneider MD, Harding SE, Redwood CS, Clarke K, Nowak K, Monserrat L, Wells D, Marston SB. J Biol Chem. 2011 Aug 5;286(31): 27582-93

[4] V. Caorsi, D.S. Ushakov, T.G. West, N. Setta-Kaffetzi, M.A. Ferenczi. Eur Biophys J. 2011; 40:13-27

Chen is a candidate for a young investigator award to be judged during the EMC 2012.

\section{0}

The fast skeletal muscle troponin activator, CK-2066260, restores the calcium-sensitivity of force development in muscle fibers from patients with nebulin-based nemaline myopathy

J.M. De Winter ${ }^{1,2}$, D. Buck ${ }^{2}$, G.J.M. Stienen ${ }^{1,3}$, J.R. Jasper ${ }^{4}$, F.I. Malik ${ }^{4}$, A.H. Beggs ${ }^{5}$, C.A.C. Ottenheijm ${ }^{1,2}$, H. Granzier ${ }^{2}$

${ }^{1}$ Insitute for Cardiovascular Research, Department of Physiology, VU University Medical Center Amsterdam, The Netherlands; ${ }^{2}$ Molecular Cardiovascular Research Program, University of Arizona, Tucson, USA; ${ }^{3}$ Faculty of Science, Department of Physics and Astronomy, VU University, Amsterdam, The Netherlands; ${ }^{4}$ Preclinical R\&D, Cytokinetics, Inc., South San Francisco, CA, USA; ${ }^{5}$ Division of Genetics and Program in Genomics, The Manton Center for Orphan Disease Research, Children's Hospital Boston, Harvard Medical School, 300 Longwood Avenue, CLSB 15026, Boston, MA 02115, USA

Nemaline myopathy (NM), the most common non-dystrophic congenital myopathy, is frequently caused by mutations in $N E B$. NEB encodes the giant protein nebulin, a major constituent of the skeletal muscle thin filament. Recent work revealed that skeletal muscle fibers from nebulin-based nemaline myopathy (NEB-NM) patients have a lower calcium-sensitivity of force development, which contributes to muscle weakness. Here, we studied the ability of a novel fast skeletal 
Table

\begin{tabular}{|c|c|c|c|c|}
\hline \multirow[t]{2}{*}{$\mathbf{p C a}_{50}($ Mean \pm SEM $)$} & \multicolumn{2}{|l|}{ SL $2.1 \mu \mathrm{m}$} & \multicolumn{2}{|l|}{ SL $2.6 \mu \mathrm{m}$} \\
\hline & Vehicle & $C K-2066260$ & Vehicle & $C K-2066260$ \\
\hline CTRL & $5.88 \pm 0.06$ & $6.11 \pm 0.04^{*}$ & $5.97 \pm 0.03^{*}$ & $6.18 \pm 0.04 * *$ \\
\hline$N E B-N M$ & $5.78 \pm 0.04^{\#}$ & $6.04 \pm 0.06^{*}$ & $5.90 \pm 0.04^{*}$ & $6.05 \pm 0.04 * *$ \\
\hline
\end{tabular}

Significantly different from ${ }^{*}$ CTRL; * SL 2.1vehicle; ** SL 2.6vehicle

muscle troponin activator, CK-2066260, to restore calcium-sensitivity in skeletal muscle fibers from $N E B$-NM patients.

Quadriceps muscle biopsies of infant NEB-NM patients $(n=4)$ were compared to age-matched controls (CTRL; $n=4)$. The effect of CK-2066260 $(5 \mu \mathrm{M})$ on maximal force generating capacity (Fmax) and calcium-sensitivity $\left(\mathrm{pCa}_{50}\right)$ was determined in skinned muscle preparations at a short (SL $2.1 \mu \mathrm{m})$ and at a longer (SL $2.6 \mu \mathrm{m})$ sarcomere length.

Fmax divided by cross sectional area of the fibers $\left(\mathrm{mN} / \mathrm{mm}^{2}\right)$ was significantly reduced in $N E B$-NM compared to CTRL at both SL $2.1 \mu \mathrm{m}(18 \pm 2$ and $86 \pm 11$, respectively) and SL $2.6 \mu \mathrm{m}(22 \pm 3$ and $99 \pm 17$, respectively). CK- 2066260 did not affect Fmax at either sarcomere length. The $\mathrm{pCa}_{50}$ data are summarized in the table below. Here, we confirmed a lower calcium-sensitivity of force development in $N E B$-NM patients compared to age- matched controls. Administration of $5 \mu \mathrm{M}$ of CK-2066260 greatly enhanced the calcium-sensitivity of force development in $N E B$-NM patients. Thus, CK-2066260, a fast skeletal muscle troponin activator, ameliorates the reduced calcium-sensitivity in $N E B$-NM and is a potential therapeutic approach in this debilitating disease.

De Winter is a candidate for a young investigator award to be judged during the EMC 2012.

\section{7}

Increased right ventricular cardiomyocyte stiffness in patients with pulmonary arterial hypertension

S. Rain ${ }^{1}$, J. van der Velden ${ }^{2}$, C.A. Ottenheijm ${ }^{2}$, M. L. Handoko ${ }^{1,2}$, N. Westerhof ${ }^{1,2}$, C.G. dos Remedios ${ }^{3}$, M. Humbert ${ }^{4}$, P. Dorfmuller ${ }^{4}$, C. Guignabert ${ }^{4}$, C. Saripalli ${ }^{5}$, H. Granzier ${ }^{5}$, A.Vonk-Noordegraaf ${ }^{1}$, F.S. de $\mathrm{Man}^{1,2}$

${ }^{1}$ VU University Medical Center, Institute for Cardiovascular Research, Department of Pulmonology; ${ }^{2}$ Department of Physiology, Amsterdam, the Netherlands; ${ }^{3}$ The University of Sydney, Department of Anatomy \& Histology; ${ }^{4}$ Paris-Sud University, INSERM U999, France; ${ }^{5}$ University of Arizona, USA

Patients with pulmonary arterial hypertension (PAH) develop severe right heart failure with extensive right ventricular (RV) remodeling. This study aims to determine whether changes at RV cardiomyocyte sarcomere level are involved in right heart failure in PAH-patients.

Human RV tissue was obtained after heart-lung transplantation of PAH-patients and non-failing donors.

Force measurements were performed on skinned RV cardiomyocytes at various $\mathrm{Ca}^{2+}$ concentrations to determine active force, passive stiffness and $\mathrm{Ca}^{2+}$ sensitivity. The role of $\beta$-Adrenergic receptor signaling on cardiomyocyte passive stiffness was mimicked by analyzing passive stiffness after incubation with endogenous protein kinase A (PKA). Protein analysis included titin isoform composition and titin phosphorylation at PKA and PKC(S26 and S170) sites.

Active force and passive stiffness were significantly increased at sarcomere lengths from 1.8 to $2.6 \mu \mathrm{m}$ in PAH patients compared to donors. PKA incubation significantly decreased RV cardiomyocytes passive tension in PAH patients to donor values. Myofilament $\mathrm{Ca} 2+$ sensitivity was significantly higher in PAH patients compared to donors. No significant changes in titin isoform composition were found, however decreased titin PKA and $\mathrm{PKC}(\mathrm{S} 26)$ phosphorylation and hyperphosphorylation of $\mathrm{PKC}(\mathrm{S} 170)$ were observed in $\mathrm{PAH}$ patients compared to donors.

$\mathrm{PAH}$-induced right heart failure was characterized by increased active force, increased calcium sensitivity and elevated RV cardiomyocyte passive stiffness. The elevated RV cardiomyocyte passive stiffness could partially be caused by altered $\beta$-adrenergic receptor signaling on PKA pathway.

\section{9}

Altered contractile function of left ventricular cardiomyocytes in patients with pulmonary arterial hypertension

E. Manders ${ }^{1}$, C.A.C. Ottenheijm ${ }^{2}$, N. Westerhof ${ }^{1}$, G.J.M. Stienen ${ }^{2}$, C. dos Remedios ${ }^{3}$, M. Humbert ${ }^{4}$, P. Dorfmüller ${ }^{4}$, J. van der Velden ${ }^{2}$, A. Vonk-Noordegraaf ${ }^{1}$, F.S. de Man $^{1}$

${ }^{1}$ VU University Medical Center, Depart. of Pulmonology and; ${ }^{2} \mathrm{VU}$ University Medical Center, Institute for Cardiovascular Research, Department of Physiology, Amsterdam, the Netherlands; ${ }^{3}$ Muscle Research Unit, Institute for Biomedical Research, The University of Sydney, Australia. ${ }^{4}$ University Paris-Sud, AP-HP, INSERM U999, Hôpital Antoine Béclère, Clamart, and Centre Chirurgical Marie Lannelongue, Le Plessis-Robinson, France

In patients with pulmonary arterial hypertension $(\mathrm{PH})$ the chronic increase in right ventricular (RV) afterload is associated with reduced left ventricular (LV) filling caused by the displacement of the interventricular septum during the diastolic phase. We hypothesized that reduced filling of the LV impairs the intrinsic contractile properties of LV cardiomyocytes.

Cardiac tissue from the LV free wall of end-stage PH-patients was obtained during heart transplantation surgery $(\mathrm{PH}, n=7)$. Non-failing cardiac LV tissue was obtained from donor hearts (DN, $n=5$ ). Cardiomyocytes were isolated and incubated for $5 \mathrm{~min}$ in relaxing solution containing $0.5 \%$ triton-X100 to permeabilize the membranes. A single cardiomyocyte was attached between a force transducer and a length motor. Maximal active tension was measured at a saturating $\mathrm{Ca}^{2+}$ concentration, while calcium sensitivity was determined at incremental $\mathrm{Ca}^{2+}$ concentrations. Passive tension was measured in relaxing solution by rapidly shortening the fiber to $70 \%$ of its length. Parameters were determined at a range of sarcomere lengths (SLs).

Maximal active tension was found to be significantly lower - at a SL of 2.2, 2.4 and $2.6 \mu \mathrm{m}$ - in LV cardiomyocytes from PH patients compared to donors (Fig. 1a). Passive tension was not different between groups at all sarcomere lengths (Fig. 1b). The $\mathrm{pCa}_{50}$, a reflection of myofilament calcium sensitivity, was not significantly different at a SL of $1.8 \mu \mathrm{m}$ (DN: $5.23 \pm 0.02$ vs PH: $5.29 \pm 0.04$, 


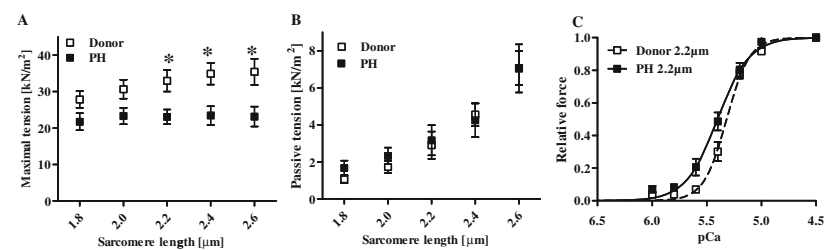

$P=0.34)$ but was significantly higher in PH patients at $2.2 \mu \mathrm{m}$ (DN: $5.32 \pm 0.02$ vs PH: $5.42 \pm 0.04, P=0.04$ ) (Fig. 1c).

These results suggest that in hearts of $\mathrm{PH}$ patients not only the RV is affected but also the LV, as demonstrated by the decrease in maximal active tension. Furthermore we found an increase of calcium sensitivity in LV cardiomyocytes from $\mathrm{PH}$ patients, which might hamper LV relaxation during diastole.

\section{0}

Nuclear magnetic resonance imaging: an efficient tool to assess the volume of individual quadriceps muscles

Y. Barnouin, N. Azzabou, D. Reversat, T. Voit, G. Butler-Brown, P.G. Carlier, J.Y. Hogrel

Institut de Myologie - GH Pitié-Salpêtrière - 75651 Paris Cedex 13 - France

Muscle volume is of the highest interest in the follow-up of pathological or aging processes. Automatic methods to detect individuals muscles are not yet reliable, so that manual segmentation, although time consuming, is still the best method to provide good delimitation between the different quadriceps components. Although interoperator has been already assessed for quadriceps volume estimation, no study has validated the reliability of manual segmentation to assess the volume of individual quadriceps muscles. The main objectives were (1) to validate the inter-operator reliability of manual segmentation to delineate individual quadriceps muscles volume using MRI and (2) to develop an efficient method to accurately assess their volume.

Twenty healthy volunteers underwent muscle MRI investigation. Continuous muscle anatomical cross-sectional areas (ACSAs) measurements along the thigh were performed every $5 \mathrm{~mm}$ using a $3 \mathrm{~T}$ MRI scanner (out of phase 3D Dixon imaging). ACSAs of the four quadriceps compartments (vastus lateralis VL, vastus intermedius VI, vastus medialis VM and rectus femoris RF) were manually segmented. Several methods were used to estimate muscle volume (cylindrical, truncated cone, 3rd and 4th order polynomial fitting methods). The effect of slice thickness on volume estimation was assessed in order to decrease the number of useful slices for the volume computation. The inter-operator reliability of manual segmentation for RF and VM was excellent but it was less for the VI and the VL because of a possible "fusion phenomenon" between these two muscles in the proximal part of the quadriceps. For muscle volume computation, $4^{\text {th }}$ order polynomial fitting seems the most robust method for estimating accurately the volumes of each of the quadriceps heads even by taking one image out of 10 . This preliminary results show that the volume of individual quadriceps muscle can be estimated in a reliable way. A consensus is still needed to standardized muscle volume assessment using MRI by defining operating procedures including imaging sequence, objective anatomical landmarks, volume modeling.

Supported by the EU Framework 7 Project MYOAGE: Understanding and Combating age related muscle weakness Grant Agreement Number: 223576.

\section{1}

\section{Structural studies of calcium regulation in $\alpha$-actinin}

E.A. Gkougkoulia ${ }^{1}$, N. Pinotsis ${ }^{1}$, B. Sjoeblom ${ }^{1}$, A. Salmazo ${ }^{1}$, B. Addario ${ }^{2}$, L. Backman ${ }^{2}$, K. Djinović- Carugo ${ }^{1}$

${ }^{1}$ University of Vienna, Dept. of Structural and Computational Biology, Max F. Perutz Laboratories, Vienna, Austria; ${ }^{2}$ Umeå University, Biochemistry, Umeå, Sweden

$\alpha$-actinin is a ubiquitously conserved protein that cross-links actin filaments. It is a member of the superfamily of proteins which also contains the spectrins and dystrophin. $\alpha$-actinins in vertebrates can be grouped into two distinct classes: non-muscle cytoskeletal calcium sensitive isoforms and muscle isoforms (calcium insensitive). Human muscle isoforms (2 and 3 ) are localized in the Z-disk and analogous dense bodies. In contrast, human non-muscle isoforms (1 and 4) are commonly associated with focal contacts and stress fibers. At evolutional level, the ancestral gene of $\alpha$-actinin encodes a calcium sensitive isoform, while the calcium insensitive forms came later with the muscle tissue development [1,2]. Nevertheless, the reason that a non-muscle cell still requires calcium regulation of the protein is still uncertain and the exact mechanism of actin binding inhibition by calcium in the non-muscle isoforms of $\alpha$-actinin is still unknown.

We selected isoform 2 of $\alpha$-actinin from Entamoeba histolytica as an archetype of an ancestral $\alpha$-actinin molecule in order to elucidate its molecular architecture, structural evolution and molecular basis of its regulation by calcium [3, 4]. 2Eh was overexpressed, purified and crystallized. Approach of using methylated sample notably enhanced its propensity to crystallize, yielding crystals diffracting to resolution of $3.5 \AA$.

\section{References}

[1] Dixson, J.D., et al., J Mol Evol., (2003), 56(1):1-10.

[2] Thomas, G.H., et al., Mol Biol Evol., (1997), 14, 1285-1295.

[3] Virel A., et al., Mol Biol Paras., (2007), 154: 82-89.

[4] Virel A., and Backman L., Mol Biochem Parasitol., (2006), 145(1):11-7.

\section{2}

Isoforms and mutations: insights into the structure, function and regulation of muscle myosins

M.A. Geeves

University of Kent, Biosciences, Molecular Motors \& the Cytoskeleton Group, Canterbury, UK

Myosins are a large family of closely related motor proteins and by studying a range of different myosin we learn about how variable function of myosins can be. We recently proposed that all myosins can be grouped into 4 types by their biochemical and functional properties (Bloemink \& Geeves (2011) Seminars in Seminars in Cell \& Developmental Biology 22, 961-967). In addition by studying one type of myosin such as the striated muscle myosin II within mammals, for example, and across wider species to include Drosophila and scallop we can begin to understand some of the detail of the structure and function of myosin and how it is adapted to particular physiological roles. The study of mutations in the myosins, both naturally occurring in myopathies, and artificially expressed mutations allows us to test some of these ideas. This lecture will explore how much we understand of myosin adaptability. In terms of regulation mammalian striated muscle myosins are regulated primarily via the troponin and tropomyosin calcium switch. Yet even here there are a variety of isoforms required to tune each regulatory system to the specific 
muscle type. What these specific requirements are (e.g. number of calcium binding sites in $\mathrm{TnC}$ ) and how they are matched to the specific myosin and muscle physiology is only just beginning to be defined.

\section{Track 2: Systems Biology \& Epigenetics}

\section{5}

Cigarette smoke stimulates muscle catabolism via p38 MAPK and muscle specific E3 ubiquitin ligases in C2 myotubes

O. Rom $^{1}$, S. Kaisari ${ }^{1}$, D. Aizenbud ${ }^{1,2}$, A.Z. Reznick ${ }^{1}$

${ }^{1}$ Technion-Israel Institute of Technology, Rappaport Faculty of Medicine, Department of Anatomy and Cell Biology, Haifa, Israel; ${ }^{2}$ Rambam Health Care Campus, Orthodontic and Craniofacial Department, Haifa, Israel

Previous epidemiological studies have identified tobacco smoking as a risk factor for sarcopenia, the age related loss of muscle mass and strength. Clinical, in vivo and in vitro studies have revealed that cigarette smoke (CS) induces skeletal muscle damage due to impaired muscle metabolism, increased inflammation and oxidative stress and activation of various intracellular signaling pathways.

In order to investigate the cellular mechanisms by which CS leads to muscle catabolism, $\mathrm{C} 2$ myotubes were exposed to different levels of whole vapor phase CS. Myotube diameters, muscle proteins degradation and activation of signaling pathways in response to CS exposure were examined by microscopy, Western blot and real time quantitative PCR.

Exposure of $\mathrm{C} 2$ myotubes to $\mathrm{CS}$ caused a reduction in myotube diameters and degradation of the main contractile proteins myosin heavy chain and actin proteins in a time- and dose-dependent manner. $\mathrm{CS}$ exposure to $\mathrm{C} 2$ myotubes also resulted in p38 MAPK phosphorylation, which led to up-regulation of the muscle specific E3 ubiquitin ligase enzymes MAFbx/atrogin-1 and MuRF1. Inhibition of p38 MAPK by SB203580 prevented both CS associated degradation of myosin heavy chain and up-regulation of the above E3 ubiquitin ligases.

Our results demonstrate that vapor phase CS exposure to skeletal myotubes activates the p38 MAPK pathway leading to skeletal muscle cell damage and muscle protein breakdown mediated by muscle specific E3 ubiquitin ligases. Our findings provide a possible molecular mechanism for the catabolic effects of CS in skeletal muscle.

\section{6}

Identification of possible cigarette smoke constituents responsible for muscle catabolism

O. Rom ${ }^{1}$, S. Kaisari ${ }^{1}$, D. Aizenbud ${ }^{1,2}$, A.Z. Reznick ${ }^{1}$

${ }^{1}$ Technion-Israel Institute of Technology, Rappaport Faculty of Medicine, Department of Anatomy and Cell Biology, Haifa, Israel; ${ }^{2}$ Rambam Health Care Campus, Orthodontic and Craniofacial Department, Haifa, Israel

Sarcopenia, the age-related loss of muscle mass and strength is significantly influenced by life style factors such as physical inactivity and impaired nutrition. Cigarette smoking is another life style habit that has been shown to be associated with sarcopenia. Smoking is prevalent worldwide and it is probably the most significant source of toxic chemicals exposure to humans. Cigarette smoke (CS) is a complex aerosol consisting of thousands of various constituents including free radicals, toxic aldehydes, etc. Recently, we have shown that vapor phase CS induces skeletal muscle catabolism via activation of p38 MAPK and up-regulation of the muscle specific E3 ubiquitin ligases MAFbx/atrogin-1 and MuRF1. In this study we aim to identify the main components of CS responsible for its catabolic effect in skeletal muscle.

C2 myotubes were exposed to acetaldehyde and acrolein, two major components of vapor phase CS that have potential catabolic effects in skeletal muscle.

Acetaldehyde exposure to $\mathrm{C} 2$ myotubes did not promote breakdown of the main contractile muscle proteins, nor did it stimulate upregulation of the muscle specific E3 ubiquitin ligases. However, acrolein exposure to $\mathrm{C} 2$ myotubes has mimicked the catabolic effects of CS and resulted in atrophy of myotubes, degradation of myosin heavy chain, activation of $\mathrm{p} 38$ MAPK and up-regulation of MAFbx/ atrogin-1 and MuRF1.

Our findings indicate that acrolein, a highly toxic $\alpha, \beta$-unsaturated aldehyde present in vapor phase CS, may be the primary component of CS responsible for CS associated catabolism of skeletal muscle.

\section{6}

Impairment of energy metabolism in senescent satellite cells is associated with oxidative modifications of specific enzymes

M.A. Baraibar ${ }^{1}$, J. Hyzewicz ${ }^{1}$, R. Ladouce ${ }^{1}$, A. Rogowska-Wrzesinska ${ }^{2}$, G. Butler-Browne ${ }^{3}$, B. Friguet $^{1}$

${ }^{1}$ Université Pierre et Marie Curie-Paris 6, UR4, IFR83, Laboratoire de Biologie Cellulaire du Vieillissement, Paris, France; ${ }^{2}$ University of Southern Denmark, Department of Biochemistry and Molecular Biology, Odense, Denmark; ${ }^{3}$ Institut de Myologie, Université Pierre et Marie Curie-Paris 6, CHU Pitié-Salpétrière, UMRS INSERM U974, CNRS UMR 7215, Paris, France

A hallmark of ageing both at the cellular and organismal level is the accumulation of damaged proteins due to increased oxidative stress and other factors. Human satellite cells constitute an interesting cellular model of ageing because they experience both chronological and replicative ageing. In addition, their replication and differentiation is compromised with age, contributing to the development of sarcopenia. However, the molecular events related to myoblasts dysfunction during ageing are not completely understood.

In this study, we provide evidence for the accumulation of oxidized proteins, as well as proteins modified by glycation and conjugated with lipid peroxidation products during replicative senescence of human myoblasts. The observed proteasome inactivation in senescent myoblasts may explain, at least in part, the accumulation of modified proteins. The modified proteins are mainly cytosolic and involved in key cellular functions. To provide mechanistic insights into the role of oxidized proteins in the development of the senescent phenotype untargeted metabolomic profiling was performed. Metabolic differences related to energy, lipid and nucleotide metabolism and catabolism of branched chain amino acids were observed. A strong correlation was found between protein modifications and impairment of the related cellular metabolic pathways. The energy metabolism impairment of senescent cells may be related to the carbonylationmediated reduction in the activity of the enzymes involved in the central metabolism.

This study establishes a new concept in relation with the impact of epigenetic changes, in particular oxidative protein modifications, on the impairment of cellular metabolism and the development of the senescent phenotype.

Supported by the EU Framework 7 Project MYOAGE: Understanding and Combating age related muscle weakness Grant Agreement Number: 223576. 
62

Optimization and application of a non-stable isotope method to measure muscle protein synthesis in vivo and ex vivo in mice F.J. Dijk ${ }^{1}$, M. Van Dijk ${ }^{1}$, J. Nagel ${ }^{1}$, Y. Luiking ${ }^{1,2}$, K. Van Norren ${ }^{1,3}$

${ }^{1}$ Nutricia Advanced Medical Nutrition, Danone Research, Centre for Specialised Nutrition, Wageningen, The Netherlands; ${ }^{2}$ Center for Translational Research in Aging \& Longevity. University of Arkansas for Medical Sciences, Little Rock, USA; ${ }^{3}$ Nutrition and Pharmacology Group, Division of Human Nutrition, Wageningen University, The Netherlands

Changes in muscle protein synthesis (MPS) are usually measured by incorporation of labeled amino acids. Recently, a novel technique was described by Schmidt et al. (2009), which uses puromycin incorporation during the translational process of protein synthesis (SUnSET) and western blot technique using a specific anti-puromycin antibody for analysis. We aimed to optimize SUnSET for ex vivo and in vivo MPS measurement in mice in the fasted and postprandial state.

For ex vivo MPS, 10-month old mice were anesthetized and tibialis anterior muscle was pre-incubated for $30 \mathrm{~min}$, followed by incubation with puromycin containing buffer of varying puromycin concentration and incubation time for method optimization. For in vivo MPS, mice were given an oral gavage of water or a mixture with high-protein to reach a postprandial state. Thirty min later $0.04 \mu \mathrm{mol} / \mathrm{g}$ puromycin were sc-injected, 45 min thereafter muscles were isolated and western blot was performed on total homogenates. Independent samples $T$ test was used for statistical analyses.

Optimal ex vivo conditions were 90 min of incubation with $10 \mu \mathrm{M}$ puromycin. Puromycin intensity was linearly correlated with protein amount. In vivo MPS significantly increased $48 \%$ in postprandial state compared with fasted state $(P=0.019)$. Plasma glucose and amino acids were significantly increased (glucose $+20 \%$; BCAA $+99 \%$; EAA $+86 \%$ ) in postprandial state.

The SUnSET method can be accurately used to measure changes in MPS both ex vivo and in vivo. In vivo MPS was enhanced in the postprandial state, which was characterized by an increase in plasma glucose and amino acid levels.

\section{8}

Insights into the molecular and cellular mechanisms involved in muscle ageing: an update from the myoage consortium

\section{A. Musarò ${ }^{1}$, V. Mouly ${ }^{2}$, G. Butler-Browne ${ }^{2}$}

${ }^{1}$ Sapienza University of Rome; DAHFMO-unit of Histology and Medical Embryology, Institute Pasteur Cenci-Bolognetti, IIM, Rome 00161, Italy; ${ }^{2}$ UPMC-Université Paris 6, Thérapie des maladies du muscle strié, Institut de Myologie, Paris, France

Sarcopenia is a universal age related loss of muscle associated with a loss of strength and function resulting in muscle weakness. It can start as early as 30 year and by 80 year $30-50 \%$ of the muscle may be lost. From previous studies it was evident that the loss in muscle mass cannot account for the quantitative differences which are observed in muscle strength. Muscle aging is characterized by a decrease in fiber size, decreased capillary density, increased fibrosis as well as a preferential loss of fast muscle fibers. At a cellular level we have shown that muscle aging is accompanied by a decrease in nuclear domain size and a decrease in the number of muscle progenitors.

Moreover, the alteration in the regenerative response represents one of the pathogenic features of muscle aging, leading to impairing physiological function and progression of sarcopenia.

Based on this evidence the goals of the Myoage project, related specifically to our workpackage, is to investigate modifications in the behavior of the muscle precursors during ageing, and determine whether they participate to muscle weakness by limiting nuclear turnover and slowing down muscle repair after trauma.

Despite numerous theories and intensive research, the principal molecular mechanisms underlying the process of muscle wasting are still unknown. Current data suggest that the development of muscle wasting is a multifactorial process resulting from both intrinsic factors, involving changes at both the molecular and cellular levels, as well as extrinsic factors such as decreased activity.

We have studied anabolic factors using transgenic mice expressing different isoforms of IGF-1, and catabolic factors (IL-6 and oxidative stress) that potentially modulate muscle mass and function. Preliminary evidences revealed a unique profile of actions for IGF-1Ea and IGF-1Eb isoforms, which was manifest at both structural and functional levels. The study underscores the significance of prepropeptides in the biology of IGF-1, and emphasizes the importance of isoform choice in considering clinical applications. Moreover, localized accumulation of oxidative stress or systemic increased of IL-6 levels are sufficient to promote several signs of sarcopenia.

In addition, the consortium involved in WP3 has now set up standard conditions that ensure reliable and informative human muscle precursor (myoblasts) cultures. Importantly, exchanges of researchers between partners has ensured an efficient transfer of technologies and protocols between partners, and a standardized protocol is now posted on the website, and has been already used for published results which ensure the dissemination of this protocol to a larger scientific community.

All of this represents an important advance in our understanding for the future treatment of muscle aging.

Supported by the EU Framework 7 Project MYOAGE: Understanding and Combating age related muscle weakness Grant Agreement Number: 223576.

\section{9}

Long-term effects of IGF-1 and/or high sarcomere strain on size and tetanic force of mature Xenopus muscle fibres

R.T. Jaspers ${ }^{1}$, J. Testerink ${ }^{1}$, R. Krishnan ${ }^{2}$, C. Offringa ${ }^{1}$, W.J. van der Laarse ${ }^{3}$

${ }^{1}$ MOVE Research Institute Amsterdam, Faculty of Human Movement Sciences, VU University Amsterdam, The Netherlands; ${ }^{2}$ Center Vascular Biology Research, Beth Israel Deaconess Medical Center, Harvard Medical School, Boston, Massachusetts, USA; ${ }^{3}$ Department of Physiology, Institute for Cardiovascular Research, VU University medical center, Amsterdam, The Netherlands

High strain imposed on a muscle in vivo may stimulate hypertrophy directly and/or indirectly by expression of insulin-like growth factor 1 (IGF-1). We investigated the separate and combined effects of high sarcomere strain and insulin-like growth factor-1 (IGF-1) on fibre cross-sectional area (FCSA), the number of sarcomere in series.

Mature, single muscle fibres of Xenopus laevis were cultured at slack length or at $112 \%$ slack length ("high strain") for 10 to 24 days in serum-free medium with or without human IGF-1.

For fibres cultured at slack without IGF-1, tetanic force and crosssectional area (CSA) did not change. Fibres cultured at high strain without IGF-1 reduced tetanic force by $1.4 \pm 0.2 \%$ (mean \pm SEM) per day, whereas CSA was constant. In contrast, tetanic force of fibres cultured at slack length with IGF-1 increased by $1.0 \pm 0.1 \% /$ day while FCSA increased by $33.4 \pm 3.8 \%$ after $16.6 \pm 0.6$ days. CSA of high strain cultured fibres with IGF-1 increased to $28.8 \pm 3.7 \%$ after $16.6 \pm 1.4$ days. The IGF-1 induced increase in tetanic force at high stain $(0.6 \pm 0.2 \%$ /day $)$ was smaller than at slack. For all conditions, numbers of sarcomeres in series and myonuclei were 
unchanged. IGF-1 increased $\alpha$-skeletal actin mRNA, whereas high strain reduced it. IGF-1 mRNA was doubled by IGF-1, but was not affected by high strain. p-Akt concentrations were independent of high strain and/or IGF-1.

We conclude that high strain imposed on an isolated muscle fibre does not stimulate hypertrophy or increase the number of sarcomeres in series, whereas IGF-1 may stimulate hypertrophic signaling via increasing IGF-1 mRNA and induce hypertrophy by increasing actin mRNA.

\section{3}

The adaptations of human skeletal muscle structure and function to unilateral lower limb suspension and retraining

E. Longa ${ }^{1}$, J. Cannavino ${ }^{1}$, L. Brocca ${ }^{1}$, O. Seynnes ${ }^{2}$, M. Narici ${ }^{3}$, M.A. Pellegrino ${ }^{1}$, R. Bottinelli

${ }^{1}$ University of Pavia, Department of Molecular Medicine, Pavia, Italy; ${ }^{2}$ Norwegian School of Sport Sciences, Oslo, Norway; ${ }^{3}$ University of Nottingham, School of Graduate Entry Medicine and Health, Derby, UK

Eight young healthy subjects were subjected to 3 weeks unilateral lower limb suspension (ULLS), a widely used human model of disuse skeletal muscle atrophy. Needle biopsies were taken from the vastus lateralis muscle pre-ULLS, post-ULLS and after 3 weeks recovery during which heavy resistance training ( $80 \%$ of $1 \mathrm{RM})$ was performed (post-ULLS-TR).

After disuse, cross sectional area (CSA), specific force (Po/CSA) and myosin concentration (MC) decreased both in type 1 and $2 \mathrm{~A}$ skinned muscle fibres. After recovery, CSA and MC returned to levels comparable to those observed before disuse, whereas Po/CSA and unloading shortening velocity (Vo) reached a higher level. Myosin heavy chain (MHC) isoform composition did not differ between experimental groups. To study the mechanisms underlying such adaptations, a 2D proteomic analysis was performed. Preliminary analysis of differentially expressed protein spots suggests disuse induced changes in: antioxidant defense systems (HspB $1, \alpha-\beta$ crystalline down-regulation and superoxide dismutase and peroxiredoxin 3 up-regulation), metabolic enzymes (triosephosphate isomerase, lactate dehydrogenase, beta enolase and creatine kinase down-regulation), myofibrillar proteins (troponin $\mathrm{T}$ and troponin I down-regulation). Post-ULLS-TR data showed an up-regulation of glycolytic (aldolase, beta enolase, glyceraldehydes-3P dehydrogenase) and oxidative enzymes (NADH dehydrogenase), and myofibrillar proteins (actin, myosin light chain, tropomyosin).

The data suggest that: a disproportionate loss of myosin compared to CSA occurs in individual muscle fibres in ULLS and can contribute to the loss of specific force in vivo; ULLS can determine a metabolic alteration, which cannot be accounted for by a shift of muscle phenotype; resistance exercise can readily counteract ULLS induced alterations.

Supported by the EU Framework 7 Project MYOAGE: Understanding and Combating age related muscle weakness Grant Agreement Number: 223576.

\section{8}

Autophagy and protein homeostasis in ageing sarcopenia

S. Carnio ${ }^{1,2,3}$, F. Lo Verso ${ }^{1,2,3}$, E. Masiero ${ }^{1,2,3}$, B. Friguet ${ }^{4}$, R. Rudolf ${ }^{5}$, R. Bottinelli ${ }^{6}$, M. Sandri ${ }^{1,2,3}$

${ }^{1}$ Dulbecco Telethon Institute, Padova, Italy; ${ }^{2}$ Venetian Institute of Molecular Medicine, via Orus, 2, 35129 Padova, Italy; ${ }^{3}$ Department of Biomedical Science, University of Padova, Padova, Italy;
${ }^{4}$ Laboratoire de Biologie Cellulaire du Vieillissement, UR4, Université Pierre et Marie Curie-Paris 6, 75252 Paris Cedex 05, France; ${ }^{5}$ Institut für Toxikologie und Genetik, Karlsruhe Institute of Technology, Karlsruhe, Germany; ${ }^{6}$ Department of Physiology and Interuniversity Institute of Myology, University of Pavia, Pavia, Italy

The ubiquitin-proteasome and autophagy-lysosome pathways are the two major routes for protein and organelle clearance. In skeletal muscle both systems are under FoxO regulation and their excessive activation induces severe muscle loss. Although altered autophagy has been observed in ageing sarcopenia and in various myopathies. the specific role of autophagy in skeletal muscle has not been determined by loss-of-function approaches.

We have generated muscle-specific autophagy-deficient mice. Autophagy deficient mice are weaker than WT and characterized by an age-dependent decrease in muscle force. Moreover abnormal and dysfunctional mitochondria keep accumulating inside myofibers, leading to an age-dependent increase of oxidative stress and, therefore, oxidized proteins. Proteomic analyses had revealed that the substrates that are preferentially carbonylated are sarcomeric proteins, and indeed, functional analyses on skinned fibers and in vitro motility assay confirmed that the acto-myosin interaction is altered in autophagy-deficient muscles. Muscle-specific deletion of autophagy also resulted in myofiber denervation with concomitant neuromuscular junction fragmentation as revealed by in vivo imaging on WT and KO animals. Treatment with antioxidant ameliorates Acetylcholine Receptor turnover, mitochondrial function and actin-myosin interaction.

Autophagy deficient mice are characterized by a precocious ageing state of skeletal muscles with ongoing oxidative stress, whose reduction can partially rescue some features of the myopathic phenotype. These findings open up new conceptual perspectives in the field of ageing sarcopenia and neuromuscular degenerative diseases.

Supported by the EU Framework 7 Project MYOAGE: Understanding and Combating age related muscle weakness Grant Agreement Number: 223576.

\section{0}

Stem cell and growth associated miRNAs are altered in COPD patients with a low fat free mass

A. Lewis ${ }^{* 1}$, A. Donaldson ${ }^{* 1,2}$, S.A. Natanek ${ }^{1}$, N.S. Hopkinson ${ }^{2}$, M.I. Polkey ${ }^{2}$, P.R. Kemp ${ }^{1}$

${ }^{1}$ Imperial College London, NHLI Molecular Medicine, London UK; ${ }^{2}$ Royal Brompton Hospital, Muscle Laboratory, London, UK; ${ }^{*}$ These authors contributed equally

Loss of fat free mass is an important co-morbidity of chronic obstructive pulmonary disease (COPD) with prognostic implications. COPD patients with a low fat free mass index (LFFMI) have reduced survival compared to patients with a normal FFMI (NFFMI). The mechanisms controlling loss of muscle mass in COPD remain unidentified.

The expression of miRNAs was analysed in the muscle and plasma of LFFMI patients compared to NFFMI patients with the same lung disease severity.

Most of the miRNAs that showed a significant difference in the muscle $(P<0.01)$ between the groups came from a single cluster on chromosome 19 (C19MC) which were all lower in the LFFMI patients. Members of this miRNA cluster were also reduced in the plasma of LFFMI patients compared to NFFMI patients. The C19MC miRNAs are reported to be expressed in stem cells and undifferentiated cells. Consistent with this the levels of miRNA expression we determined were very low suggesting that expression was restricted to a subset of cells. PCR in a larger group for two C19MC miRNAs 
showed an inverse correlation of expression with FFMI. The plasma of LFFMI patients also contained less miR-483 an miRNA produced from the IGF-2 gene. Interestingly miR-675 showed the largest increase in LFFMI patients compared to NFFMI patients and this miRNA is produced from $\mathrm{H} 19$ non-coding RNA gene that suppresses IGF-2 transcription.

These data suggest that LFFMI patients have reduced stem cell number or activity and reduced expression of growth modulators compared to NFFMI patients.

129

Absence of the Na, K-ATPase $\alpha 2$ isoform in gene-targeted mice

T. Radzyukevich ${ }^{1}$, N. Oshiro ${ }^{2}$, J. Neumann ${ }^{2}$, T. Rindler ${ }^{2}$, J. Lingrel ${ }^{2}$, J. Heiny ${ }^{1}$

${ }^{1}$ University of Cincinnati, Molecular and Cellular Physiology, OH USA; ${ }^{2}$ Molecular Genetics, Cincinnati OH USA

The Na,K-ATPase $\alpha 2$ isoform is the major isoform of adult skeletal muscles (Orlowski \& Lingrel 1988). This distribution is unique compared to most other tissues which express mainly the $\alpha 1$ isoform, but its physiological significance is not known. Recent studies suggest that the functional role(s) of the $\alpha 2$ isoform becomes apparent during muscle activity (Radzyukevich et al. 2009).

To further study its physiological function, we generated a novel gene-targeted mouse $\left(\mathrm{sk} \alpha 2^{-/-}\right)$which specifically lacks the $\mathrm{Na}$, K-ATPase $\alpha 2$ in skeletal muscle fibers, while retaining its normal distribution in heart, brain, vasculature, and peripheral nerves (as verified by Western blot and immunocytochemistry). The sk $\alpha 2^{-l-}$ mice are apparently normal under basal laboratory conditions but show greatly reduced exercise capacity when challenged with treadmill running. EDL muscles stimulated in vivo via the motor nerve are intolerant to fatigue and unable to maintain force during a prolonged tetanus. Similar contractile impairments are seen on isolated EDL muscles.These results indicate that the contractile impairment of $\mathrm{sk} \alpha 2^{-l-}$ muscles originates in the skeletal muscles, and not from any change in systemic factors which contribute to exercise performance in the animal. Additionally, sk $\alpha 2^{-1-}$ EDL and SOL muscles produce lower specific twitch and tetanic force. The $\mathrm{Na}$, K-ATPase $\alpha 1$ isoform, despite being upregulated in $\mathrm{sk} \alpha 2^{-/-}$muscle, cannot compensate for the absence of $\alpha 2$.

The results demonstrate that stimulated active transport by the $\alpha 2$ isoform plays an essential, isoform-specific role in maintaining contractile performance, contributing to muscle strength and fatigueresistance.

Supported by the Physiology Research Fund and the NIH.

\section{1}

\section{Catecholamines prevent additional proteolysis in Soleus} but not in EDL muscle of rats exposed to 24-hour cold

L.H. Manfredi, N. Zanon, E. Filippin, M.A. Garófalo, L.C.C. Navegantes, I.C. Kettelhut

University of São Paulo, School of Medicine of Ribeirão Preto, Department of Physiology and Department of Biochemistry and Immunology, São Paulo, Brazil

We have previously shown that catecholamines exhibit an inhibitory tonus on protein degradation in rat skeletal muscle. In order to study the role of catecholamines in protein degradation of soleus and extensor digitorum longus (EDL) "in vivo", we used in the present work a model in which the sympathetic nervous system activity is increased by cold exposure. For this, Wistar male rats $(\sim 80 \mathrm{~g})$ were submitted to adrenodemedullation (ADM) and then exposed to $24 \mathrm{~h}$ in a cold room at $4{ }^{\circ} \mathrm{C}$.

After $12 \mathrm{~h}$ of cold, rats showed an increase by $31 \%$ in plasma adrenaline, however no changes were observed after $24 \mathrm{~h}$. In this latter period of cold, normal rats exhibited an increase of $37 \%$ in overall proteolysis in soleus and $17 \%$ in EDL when compared to controls $\left(25^{\circ} \mathrm{C}\right)$. ADM rats exposed to cold exhibited an additional increase in overall proteolysis by $15 \%$ only in soleus (nmol of tyrosine $\mathrm{mg}^{-1} 2 \mathrm{~h}^{-1}$ ) when compared to normal rats exposed to $4{ }^{\circ} \mathrm{C}$ $(0.625 \pm 0.018$ vs. $0.541 \pm 0.018)$. This additional increase was due to the increase in $63 \%$ in the activity of calcium dependent system without any changes in lysosomal and ubiquitin-proteasome systems.

The acute exposition of homeothermic animals to low temperatures promotes an increase in catabolic hormones, such as corticosterone and thyroid hormones, which play a dominant role in skeletal muscle leading to an increase in proteolysis. On the other hand, catecholamines seem to prevent the loss of protein content from oxidative muscle in this condition by inhibiting the calcium dependent proteolysis.

\section{0}

Disruption of skeletal muscle mitochondrial network genes and miRNAs in amyotrophic lateral sclerosis

A.P. Russell ${ }^{1}$, S. $\mathrm{Wada}^{2}$, L. Vergani ${ }^{3}$, S. Lamon ${ }^{1}$, B. Léger ${ }^{4}$, T. Ushida ${ }^{2}$, R. Cartoni ${ }^{4}$, G. Wadley ${ }^{1}$, P. Hespel $^{5}$, G. Soraru ${ }^{3}$, C. Angelini ${ }^{3,}$ T. Akimoto ${ }^{2}$

${ }^{1}$ Deakin University, School of Exercise and Nutrition Sciences, Centre for Physical Activity and Nutrition, Melbourne, Australia; ${ }^{2}$ The University of Tokyo, Division of Regenerative Medical Engineering, Center for Disease Biology and Integrative Medicine, Tokyo, Japan; ${ }^{3}$ University of Padua, Neurosciences Department, Padua, Italy; ${ }^{4}$ Clinique Romande de Réadaptation, Sion, Switzerland; ${ }^{5}$ K. U. Leuven, Faculty of Kinesiology and Rehabilitation Sciences Research Centre for Exercise and Health, Leuven, Belgium

Skeletal muscle mitochondrial dysfunction plays a role in amyotrophic lateral sclerosis (ALS). PGC- $1 \alpha$ signalling and miRNAs are involved in mitochondrial biogenesis and muscle function. The regulation of PGC- $1 \alpha$ and miRNAs have not been investigated in skeletal muscle of ALS patients. This study measured, in vastus lateralis muscle samples from healthy control subjects $(n=10)$, patients with ALS $(n=14)$ and patients with neurogenic disease (ND) $(n=10),(1)$ expression levels of PGC- $1 \alpha$ and several downstream targets involved in mitochondrial biogenesis and function; (2) mitochondrial enzyme activity and (3) expression levels of selected miRNAs. A functional protein/miRNA relationship was also investigated in vitro using reporter assays and in vivo using miR-23a transgenic mice.

When compared with healthy control subjects, patients with ALS had reduced levels of PGC- $1 \alpha$ mRNA and protein and ERR $\alpha$ and Mfn2 mRNA. NRF-1 and Mfn1 mRNA as well as COXIV mRNA and protein, as well as citrate synthase and COX activities, were also significantly lower in patients with ALS and ND. miRNA 23a, 29b, 206 and 455 were increased in skeletal muscle of ALS patients. miR23a repressed PGC- $1 \alpha$ translation in a $3^{\prime}$ UTR dependent manner. Mice over expressing miR-23a also had a reduction in PGC- $1 \alpha$, cytochome-b and COXIV protein levels.

Skeletal muscle mitochondrial dysfunction in ALS patients is associated with reduced PGC- $1 \alpha$ and several of its targets involved in mitochondrial biogenesis and function, as well as increases in miRNAs potentially implicated in muscle and neuromuscular junction regeneration. miR-23a directly targets and reduces muscle PGC- $1 \alpha$ protein levels, and impairs muscle mitochondrial function. The 
increase in miR-23a and associated decrease in PGC- $1 \alpha$ protein levels in ALS potentially has a causal effect on muscle mitochondrial dysfunction. miR-23a may be a therapeutic target to ameliorate muscle function and pathogenesis of ALS.

\section{1}

Skeletal muscel plasticity in response to mechanical loading and heat stress in heat shock transcription factor 1-null mice

T. Yoshioka ${ }^{1}$, Y. Ohno ${ }^{2}$, A. Goto ${ }^{2}$, A. Ikuta ${ }^{2}$, T. Ohira ${ }^{2}$, M. Suzuki ${ }^{2}$, T. Egawa ${ }^{2,3}$, K. Yasuhara ${ }^{4}$, T. Koya ${ }^{4}$, S. Nishizawa ${ }^{4}$, A. Nakai ${ }^{5}$, S. Takao ${ }^{6}$, Y. Ohira ${ }^{7}$, K. Goto ${ }^{2}$

${ }^{1}$ Hirosaki Gakuin University; Hirosaki; ${ }^{2}$ Dept. Physiol., Graduate Sch. Med., Toyohashi SOZO Univ., Toyohashi; ${ }^{3}$ JSPS Research Fellow; ${ }^{4}$ Dept. Orthop. Surg., St. Marianna Univ. Sch. Med., Kawasaki; ${ }^{5}$ Dept. of Biochem. Mol. Biol., Grad. Sch. Med.; ${ }^{6}$ Exerc. Health Sci., Yamaguchi Univ., Yamaguchi; ${ }^{7}$ Grad. Sch. Med., Osaka Univ., Toyonaka, Japan

Loading-associated mechanical stress causes an increase in the expression of heat shock proteins (HSPs) in skeletal muscles via stress response. Application of heat- stress also up-regulates HSPs in skeletal muscles. However, a physiological role of stress response in skeletal muscle is still unclear.

In the present study, by using the heat shock transcription factor 1-null (HSF1-null) mice, we examined a role of stress response in the skeletal muscle plasticity in mice. Mechanical stress and heat-stress were applied by the reloading on soleus following 2-week-hindlimbsuspension and by the exposure to heat environment $\left(41^{\circ} \mathrm{C}\right)$ for $60 \mathrm{~min}$, respectively. Both mRNA and protein expression levels of HSPs were increased by mechanical stress, but not heat-stress in HSF1-null mice.

Loading-associated stress response may be different from that induced by heat-stress.

This study was supported, in part, by KAKENHI (22240071, TY; 24650411, KG) from Japan Society for the Promotion of Science, and Mutual Aid Corporation for Private Schools of Japan (KG).

\section{4}

The contraction duration is not important for hypertrophy in strength trained rats

E.E.H. Hansen ${ }^{1}$, J.C. Bruusgaard ${ }^{1,2}$, K. Gundersen ${ }^{1}$

${ }^{1}$ University of Oslo, Department of Molecular Biosciences, 0371 Oslo, Norway; ${ }^{2}$ Atlantis Medical College, Kongsveien 30, 0193 Oslo, Norway

It is believed that time-under-tension is an important factor for producing hypertrophy. We have performed a direct comparison between a long (300 pulses) and a short (10 pulses) isometric contraction, with the total number of action potentials being identical in the two groups.

The extensor digitorum longus (EDL) and tibialis anterior (TA) muscle of the right leg were stimulated through the peroneal nerve with a frequency of $100 \mathrm{~Hz}$ to produce tetanic isometric contractions, and the mechanical output were measured using an in vivo Muscle Test System (Aurora Scientific). The left leg was used as a sham operated control. Two different stimulation patterns were used, one with a single train of 300 pulses (long train) for each contraction, the other with 30 consecutive train pulses with 10 pulses in each train, starting every $400 \mathrm{~ms}$ (short train). Both groups received 6 sets with 6 repetitions per set, resulting in the same total amount of pulses and training time for both groups. After 6 weeks, the muscles were taken out for in vitro force measurements (EDL) (Aurora Scientific) and further histological analyses (EDL and TA).

Preliminary data show that both the short train- and the long trainregime lead to an increase in force $(10 \%)$, muscle weight $(\mathrm{EDL}=10 \%, \mathrm{TA}=16 \%)$ and fiber cross sectional area $(25 \%)$, with no significant differences between the short train and long train group.

These results suggest that the total number of impulses or the accumulated time-under-tension is important, rather than how the tension or pulses are distributed over time.

\section{6}

The role of $\mathrm{uPa} / \mathrm{TGF}-\beta 1$ bioregulation system in the regulation of eccentric exercise induced muscle damage

A. Theos ${ }^{1,2}$, M. Maridaki ${ }^{2}$, A. Philippou ${ }^{1}$, A. $\operatorname{Nezos}^{1}$, C. Karatzaferi ${ }^{3}$, M. Koutsilieris ${ }^{1}$

${ }^{1}$ National and Kapodistrian University of Athens, Medical School, Department of Experimental Physiology, GR; ${ }^{2}$ National and Kapodistrian University of Athens, Faculty of Physical Education \& Sport Science, Department of Sports Medicine \& Biology of Physical Activity, GR; ${ }^{3}$ University of Thessaly, Department of Physical Education \& Sport Science, GR

In contrast with isometric exercise, high intensity eccentric exercise causes muscle damage, which is followed by the regenerationremodelling process of the damaged muscle. The purpose of the present study was to compare the expression responses of urokinasetype plasminogen activator (uPA), its receptor (uPA-R), transforming growth factor- $\beta 1$ (TGF- $\beta 1$ ) and insulin-like growth factor binding protein-3 (IGFBP3), which are thought to be involved in the regeneration process, after two different types of exercise that are expected to cause or not cause muscle damage.

Six male volunteers (age: $26.0 \pm 1.6$ years, height: $178.3 \pm$ $1.6 \mathrm{~cm}$, mass: $77.2 \pm 2.5 \mathrm{~kg}$ ) performed on one occasion 50 maximal eccentric muscle actions using the knee extensor muscles, which resulted in muscle damage. Muscle biopsy samples were collected before and $6 \mathrm{~h}$ post-exercise. On another occasion, at least sixteen days apart, subjects performed a bout of non-damaging isometric exercise and $6 \mathrm{~h}$ later a muscle biopsy sample was also obtained. The mRNA expression changes of uPA, uPA-R, TGF $\beta 1$ and IGFBP3 were investigated by real-time PCR and compared using $T$ test.

Significantly increased expression of uPA-R, uPA, TGF $\beta 1$ and IGFBP3 was found following only eccentric exercise compared to pre-exercise expression levels $(P<0.05-0.01)$. Isometric exercise did not induce significant changes in the expression of any of the genes tested.

The uPA-mediated activation of latent-TGF $\beta 1$ and IGF-I bioavailability probably regulate skeletal muscle regeneration events of exercise-induced damage.

Theos is a candidate for a young investigator award to be judged during the EMC 2012.

\section{5}

Protein expression, fiber type composition and fatigue in the fast skeletal muscles of chronic undernourished rats

A. Ruiz-Rosado ${ }^{1}$, H. Cabrera-Fuentes ${ }^{3,5}$, C. González-Calixto ${ }^{2}$, L. González-López ${ }^{2}$, F. Fernández-Valverde ${ }^{6}$, F.E. Cázares-Raga ${ }^{2}$, B. Segura-Alegria ${ }^{4}$, G. Lochnit ${ }^{3}$, K.T. Preissner ${ }^{3}$.

F. Hernández- Hernández ${ }^{2}$, I. Jiménez Estrada ${ }^{1}$

${ }^{1}$ CINVESTAV, IPN. Department of Physiology, Biophysics and Neurosciences, Mexico City, Mexico; ${ }^{2}$ CINVESTAV, IPN. 
Department of Infectomic and Molecular Pathogenesis, Mexico City, Mexico; ${ }^{3}$ Justus-Liebig-University of Giessen. Institute for Biochemistry, Medical Faculty, Giessen, Germany; ${ }^{4}$ FES Iztacala, UNAM. Department of Biology. Mexico City, Mexico; ${ }^{5}$ Kazan Federal University, Department of Microbiology, Faculty of Biology and Soils, Kazan, Russia; ${ }^{6}$ National Institute of Neurology and Neurosurgery "Manuel Velasco Suárez", Department of Experimental Pathology, Mexico City, Mexico.

Inadequate food consumption leads to considerable alterations in the morphological, biochemical and functional properties of skeletal muscles.

In this study we analyzed the influence of chronic undernutrition on the protein expression, fiber type composition and fatigue in the extensor digitorum longus (EDL) muscle of young Wistar rats. Protein profiles from EDL muscles of control (C) and undernourished (U) male Wistar rats ( $45 \pm 3$ days of life) were generated by $2 \mathrm{DE}$ and analyzed by MS, histoenzymatic characterization of muscle fiber types and in vitro muscle fatigue analysis. Key enzymes that participate in the electron transport chain and glycolytic pathway, and the cross bridge cycle proteins showed over-expression, in addition 2 proteins involved in contractile mechanisms of fast fibers showed down-expression. In NADH-TR and COX stained sections, U-EDL muscles showed a higher proportion of oxidative fibers $(111 \%$ and $110 \%$, respectively) than C-EDL muscles. In mATPase stained sections of U-EDL muscles contained a larger proportion of type IIA/ IID muscle fibers $(115 \%)$ than C-EDL sections. The fatigue evoked by repetitive low-frequency stimuli trains $(30 \mathrm{~Hz})$ in U-EDL muscles was reached at a longer time $(157 \%)$ than in C-EDL muscles.

These results indicate that adaptive and/or compensatory mechanisms may contribute to counterbalance the imposed food deprivation and to maintain the biochemical and physiological requirements of skeletal fast muscles in the rat.

\section{6}

Effect of aging on muscle force-generating capacity and fragility in female dystrophic mdx mouse

C. Hourdé ${ }^{1,2}$, P. Joanne ${ }^{3}$, O. Agbulut ${ }^{3}$, G. Butler-Browne ${ }^{1,2}$, A. Ferry ${ }^{1,2}$

${ }^{1}$ Université Pierre et Marie Curie-Paris6, UMR S794, INSERM, U974, CNRS UMR7215, Institut de Myologie, Paris, France; ${ }^{2}$ MyoAge (EC 7th FP, contract 223576); ${ }^{3}$ Université Paris Diderot, CNRS EAC 4413, Unit of Functional and Adaptive Biology, Paris, France

Dystrophic features in hindlimb skeletal muscle from aged female mdx mice are unclear. We analysed force-generating capacity and force drop following lengthening contraction-induced damage (fragility).

Young (6 month old) female mdx mice displayed a reduced forcegenerating capacity $(-18 \%)$ and higher fragility (23\% force drop) as compared to female age-matched wild-type mice. These two dystrophic features were less accentuated in young female than in young male mdx mice ( $-32 \%$ and $42 \%$ force drop). With advancing age, force-generating capacity decreased and fragility increased in old (20 month old) female mdx mice ( $-21 \%$ and $57 \%$ force drop), but were unchanged in old male mdx mice. Moreover, estradiol treatment had no effect in old female mdx mice.

Female-related factors mitigate dystrophic features in the young but not old mdx mice. Further studies are warranted to identify the female related factor that is beneficial for the dystrophic muscle.

Supported by the EU Framework 7 Project MYOAGE: Understanding and Combating age related muscle weakness Grant Agreement Number: 223576.

\section{Track 3: Clinical \& Technological Transfer}

39

The p97/VCP ATPase is critical in myofibrillar protein degradation and muscle atrophy

R. Piccirillo ${ }^{1,2}$, A.L. Goldberg ${ }^{1}$

${ }^{1}$ Harvard Medical School, Department of Cell Biology, Boston, MA 02115, USA; ${ }^{2}$ Istituto di Ricerche Farmacologiche "Mario Negri”, Oncology Department, Via La Masa 19, 20156, Milan, Italy

The p97/VCP ATPase complex facilitates the extraction and degradation of ubiquitinated proteins from larger structures. We therefore studied if $\mathrm{p} 97$ is critical in the degradation of myofibrillar proteins during muscle atrophy. Electroporation of a dominant negative p97 (DNp97), but not the WT, into mouse muscle reduced fiber atrophy caused by denervation and food deprivation. DNp97 (acting as a substrate-trap) became associated with specific myofibrillar proteins and its cofactors, Ufd 1 and $\mathrm{p} 47$, and caused accumulation of ubiquitinated components of thin and thick filaments. Thus, p97 appears to extract ubiquitinated proteins from myofibrils during atrophy. DNp97 expression in myotubes reduced overall proteolysis by proteasomes and lysosomes and blocked the accelerated proteolysis induced by FoxO3, which is essential for atrophy. Expression of p97, Ufd1 and p47 increases following denervation, at times when myofibrils are rapidly degraded. Surprisingly, p97 inhibition, though toxic to most cells, caused rapid growth of myotubes (without enhancing protein synthesis) and hypertrophy of adult muscles.

Thus, p97 restrains post-natal muscle growth, and during atrophy, is essential for the disassembly and degradation of the contractile apparatus.

\section{2}

Myopathies associated with B-tropomyosin mutations: a review illustrated with a new case

H. Tajsharghi ${ }^{1}$, M. Ohlsson ${ }^{1}$, L. Palm ${ }^{2}$, A. Oldfors ${ }^{1}$

${ }^{1}$ Department of Pathology, Institute of Biomedicine, University of Gothenburg, Sahlgrenska Hospital, 41345 Gothenburg, Sweden; ${ }^{2}$ Department of Paediatrics Malmoe, Skane University Hospital, 20502 Malmoe, Sweden

Mutations in TPM2, encoding $\beta$-tropomyosin, have recently been found to cause a range of muscle disorders. We review the clinical and morpholgical expression of the previously reported mutations and describe an additional case with a novel mutation illustrating the clinical and morphological heterogeneity of $\beta$-tropomyosin associated diseases.

The manifestations of mutations in TPM2 include nonspecific congenital myopathy with type 1 fiber predominance, nemaline myopathy, cap disease and distal arthrogryposis. In addition Escobar syndrome with nemaline myopathy is a manifestation of homozygous truncating $\beta$-tropomyosin mutations.

Cap disease appears to be the most common morphological manifestation. A coarse inter myofibrillar network and jagged Z-lines are additional common morphological changes. The manifestations of the dominant $\beta$-tropomyosin mutations are either a congenital myopathy or distal arthrogryposis. The various congenital myopathies are usually associated with moderate muscle weakness and no congenital joint contractures. The distal arthrogryposis manifestations include the milder forms with congenital contractures mainly of hands and feet and no or mild muscle weakness.

In conclusion, there are no pathognomonic features associated with TPM2 mutations but $\beta$-tropomyosin defects should be 
considered in distal arthrogryposis and in congenital myopathies especially in cap disease but also nemaline myopathy and in congenital myopathy with unspecific morphological alterations.

\section{5}

Over-expression of ribonucleotide reductase to enhance function of normal and infarcted hearts

S.G. Nowakowski ${ }^{1}$, S.C. Kolwicz ${ }^{2}$, G.L. Odom ${ }^{3}$, S. Lundy ${ }^{1}$, F.S. Korte ${ }^{1,4}$, S.D. Hauschka ${ }^{5}$, J.S. Chamberlain ${ }^{3}$, R. Weiss ${ }^{6}$, R. $\operatorname{Tian}^{1,2,4}$, M. Regnier ${ }^{1,4}$

${ }^{1}$ University of Washington, Department of Bioengineering, Seattle, WA 98195 USA; ${ }^{2}$ University of Washington, Department of Anesthesiology, Seattle, WA 98195 USA ${ }^{3}$ University of Washington, Department of Neurology, Seattle, WA 98195 USA;

${ }^{4}$ University of Washington, Center for Cardiovascular Biology, Institute for Stem Cell \& Regenerative Medicine, Seattle WA 98109 USA; ${ }^{5}$ University of Washington, Department of Biochemistry, Seattle, WA 98195 USA; ${ }^{6}$ Cornell University, Department of Biomedical Sciences, Ithaca, NY 14853 USA

We have previously demonstrated that 2 deoxy-ATP (dATP) increased the magnitude and rate of force production in demembranated cardiac muscle via increased crossbridge binding and cycling rates. Furthermore, we demonstrated that cellular [dATP] is increased by overexpression of ribonucleotide reductase (R1R2), and this increases contractility in adult rat cardiomyocytes from normal or infarcted hearts.

Here we studied elevated cardiac R1R2 in vivo by1) injection of adeno-associated virus (AAV6) to drive R1R2 production using a cardiac specific promoter (AAV6-R1R2 $2^{\mathrm{cTnC} 4551}$ ) and 2) in transgenic mice (Tg-R1R2). Left ventricle (LV) fractional shortening was significantly greater in 3- and 6-month old Tg-R1R2 animals, compared to wild-type (WT) littermates, and enhanced for at least 6 months in AAV6-R1R2 injected mice. LV pressure development (balloon catheterization) was elevated in Tg-Rrm vs. WT Langendorff perfused hearts, and concurrent NMR spectra indicated only a slight reduction of basal PCr:ATP ratio and no difference during high workload challenge. When AAV6-R1R2 was injected into infarcted adult rat hearts, FS returned to control (un-infarcted) levels within 2 weeks. Isolated cardiomyocytes with elevated R1R2 had almost doubled magnitude of shortening, as well as faster contraction and relaxation. There was no change in $\mathrm{Ca}^{2+}$ transient amplitude but the rate of transient decay was increased. RT-PCR and western blot analysis confirmed R1R2 over-expression. Cellular [dATP] was determined to be $1-2 \%$ of the cardiomyocyte adenine nucleotide pool via HPLC, and $\sim 10$-fold increase over control cells.

These experiments demonstrate that methods to directly targeting crossbridge can enhance contractility without impairing relaxation in normal and infarcted hearts.

\section{7}

Eccentric exercise: a model for testing sensitivity to inflammation and regenerative capacity of skeletal muscle

\section{K.H. Myburg, M. van de Vyver}

Stellenbosch University, Department Physiological Sciences, Stellenbosch, South Africa

Inactivity and lack of systematic exercise training results in development of skeletal muscle weakness and possibly inflammatory conditions such as metabolic syndrome. Although chronic inflammation is easily assessed using routine chemistry, it is not clear how to determine a propensity to develop sustained inflammation in otherwise healthy individuals. We aimed to evaluate inflammatory responses in blood and muscle samples after downhill running (DHR).

Twenty-one young healthy, but untrained males, participated in a high intensity intermittent, DHR protocol. The microdamage experienced was sufficient to activate satellite cell proliferation. A unique aspect of our studies using this protocol is the ability to identify a subset of subjects who experience, at 3 to 4 days post-DHR, a second rise in circulating markers of muscle damage (DHR2), while others return to normal levels (DHR1). Circulating IL-6 concentration, [IL-6], was similarly elevated in both groups immediately post-exercise, but only DHR2 still had significantly elevated [IL-6] $4 \mathrm{~h}$ post-DHR. Phosphorylation of signal transducer and activator of transcription (STAT)-3 was higher in muscle of DHR2 subjects $4 \mathrm{~h}$ post-DHR and correlated with plasma [IL-6] at the same time point. Although neutrophils were similarly elevated $4 \mathrm{~h}$ post-DHR in both groups, only DHR2 had significantly elevated intramuscular myeloperoxidase (MPO) 2 days post-DHR. There was an association between creatine kinase acitivity and MPO.

Young healthy individuals, exposed to muscle microdamage from unaccustomed eccentric exercise, can be divided into those with a competent and those with a less efficient capacity to control postdamage inflammatory processes.

\section{0}

\section{SERCA1b EXPRESSION IN HUMAN NEONATAL MUSCLES}

M. Kósa ${ }^{1}$, K. Brinyiczki ${ }^{2}$, E. Zádor ${ }^{1}$

${ }^{1}$ Department of Biochemistry, Faculty of General Medicine, University of Szeged, Hungary; ${ }^{2}$ Department of Pathology, Faculty of General Medicine, University of Szeged, Hungary

The sarcoplasmic reticulum calcium ATPase, SERCA1 has two isoforms: SERCA1a (in adult fast fibres) and SERCA1b (in neonatal/ regenerating myotubes). The two isoforms are created by alternative splicing. The penultimate exon containing the first translation stop is skipped in the SERCA1b mRNA and a DEDPERRK C-terminal is translated instead of a single glycin of the SERCA1a protein. Using antibody against the C-terminal octapeptide tail and a pan SERCA1 antibody, our group previously described that SERCA1b was expressed only in regenerating muscle and muscle cell culture. Muscle regeneration recapitulates neonatal development therefore we investigated SERCA1b protein expression in post mortem human neonatal muscles.

Samples were taken from 4 different muscles of 5 subjects (including intrauterine exits to 8 weeks old newborns). Sarcoplasmic protein fractions were used for Western blotting, and immunohistochemistry was done on frozen sections.

SERCA1b protein was detected on Western blots regardless of age and muscle type. In contrast to regenerating rat soleus, where all fibres showed intense positivity, only certain fibres stained with SERCA1b on neonatal human sections. The majority of these fibres did not stain for panSERCA1 on consecutive sections.

We conclude that human late foetal and neonatal muscles express SERCA1b which can not be unambiguously revealed by immunohistochemistry based on the available SERCA1b antisera; only confirmed with the panSERCA1 antibody.

\section{0}

The $\mathrm{Rag}^{-} \mathrm{Il2} \mathrm{rb}^{-} \mathrm{Dmd}^{-}$mouse model: a novel model to assess cell-therapy strategies for muscular dystrophy

D. Vallese $e^{1,2,3,4}$, E. Negroni ${ }^{1,2,3}$, A. Ferry ${ }^{1,2,3}$, A. Aamiri ${ }^{5}$, J. Di Santo ${ }^{6}$, L. Vitiello ${ }^{4}$, V. Mouly ${ }^{1,2,3}$, G. Butler-Browne ${ }^{1,2,3}$ 
${ }^{1}$ Institut de Myologie, UPMC Université Paris 6, UM76, 47 bd de l'Hôpital, 75651 Paris, France ; ${ }^{2}$ INSERM U 974, Institut de Myologie, 47 bd de l'Hôpital, 75651 Paris, France $;{ }^{3}$ Institut de Myologie, CNRS UMR 7215, 47 bd de l'Hôpital, 75651 Paris, France $;{ }^{4}$ University of Padova, Department of Biology, Via Ugo Bassi 58/B, 35131 Padova, Italy; ${ }^{5}$ Université Ibn Zohr, Laboratoire de Biologie cellulaire et moléculaire, Faculté des Sciences, 80000 Agadir- Maroc ; ${ }^{6}$ Institut Pasteur, Cytokines and Lymphoid Development Unit, INSERM U 668, Paris, France

Cell transplantation is considered as a potential therapeutic approach to muscular dystrophies, however, it still requires deeper insights into the in vivo behaviour of transplanted human myogenic cells, for which an optimized model would be immunodeficient and dystrophic. This study focuses on the characterization of a new immunodeficient and dystrophic $\mathrm{Rag}^{-} \mathrm{Il} 2 \mathrm{rb}^{-} \mathrm{Dmd}^{-}$mouse model that we have recently developed.

We measured the whole body weight and the weight of different leg muscles. We measured the serum levels of creatine kinase (CK), and the regeneration process was quantified by immunohistochemical analysis of neonatal myosin heavy chain. We also performed physiology tests and assessed the feasibility of injecting human myoblasts.This dystrophic model showed growth curves similar to $m d x$ (total body and single muscles weight). In $m d x$ mice, $\mathrm{CK}$ levels showed a significant decrease between 8 and 24/40 weeks of age, whereas $\mathrm{Rag}^{-} \mathrm{Il} 2 \mathrm{rb}^{-} \mathrm{Dmd}^{-}$ showed an opposite trend. Rag2 $2^{-} I l 2 r b^{-} D m d^{-}$animals present a wider and delayed regeneration peak, which started between 10 and 16 weeks of age. Both dystrophic models presented similar decreases in the specific force. Transplantation of human myoblasts were successful in Rag2 ${ }^{-} I l 2 r b^{-}{ }^{-} m d^{-}$animals.

The muscular phenotype of this new mouse model clearly resembles that of the $m d x$ model, and therefore constitutes a useful tool that can be used for assessing gene and cell therapy approaches, particularly using human myogenic stem cells.

\section{1}

\section{Uropathogenic Escherichia Coli (UPEC)-mediated impairment} of ureteric contractility

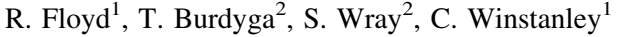

${ }^{1}$ University of Liverpool, Department of Clinical Infection, Microbiology and Immunology, Institute of Infection and Global Health, Liverpool, UK; ${ }^{2}$ University of Liverpool, Department of Cellular and Molecular Physiology, Institute of Translational Medicine, Liverpool, UK

Most urinary tract infections (UTI) are caused by UPEC. The ureters play a key role in keeping the kidneys free from infection. Previously, we showed that two well-studied UPEC strains (J96 and 536) can subvert this role, leading to reduced ureter contractility in a rat model. This study determined whether this effect is (1) a widespread feature of UPEC and (2) exhibited only by UPEC, by analysing UPEC strains representing epidemiologically important multilocus sequence types ST131, ST73 and ST95, and representative enteropathogenic and avian pathogenic E. coli.

Changes in phasic contractions and $\mathrm{Ca}^{2+}$ transients evoked by electrical field stimulation of Indo-1 loaded rat ureters during exposure to UPEC were investigated.

Five of the six UPEC strains caused a significant reduction in phasic contractions and $\mathrm{Ca}^{2+}$ transients whereas non-UPEC strains had no effect. UPEC strains exhibiting the most potent effects on contractility (CFT073 and UTI89) were inhibited by mannose but there were variations amongst other UPEC strains tested. Using mutation and complementation studies, we demonstrated that the UTI89 effects were dependent upon fimH.

Our study indicates that although the ability to impact on ureter contractility is a widespread feature of UPEC, the mechanisms involved may vary between strains.

\section{7}

Actomyosin and nanotechnology-from fundamental studies to ultrafast nanoseparation and biosensing

A. Månsson ${ }^{1}$, S. Kumar ${ }^{1}$, M. Lard ${ }^{2}$, L. ten Siethoff ${ }^{1}$, M. Persson ${ }^{1}$, N. Albet-Torres ${ }^{1}$, G. te Kronnie ${ }^{3}$, H. Linke ${ }^{2}$

${ }^{1}$ Linnaeus University, School of Natural Sciences, SE-391 82 Kalmar, Sweden; ${ }^{2}$ Lund University, The Nanometer Structure Consortium (nmC@LU) \& Div Solid State Physics. SE-22100 Lund, Sweden; ${ }^{3}$ University of Padua, Dept. Pediatrics "Salus Pueri”, SSD Clinical and Experimental Hematology, 35128 Padova Italy

In lab-on-a-chip systems, bioanalytical steps from separation to detection of an analyte (e.g. a disease biomarker) occur on a single chip. However, the developments have been hampered by the requirement of bulky accessory equipment for powering and controlling the process. Here, molecular motor driven transportation has a considerable potential. In the gliding in vitro motility assay, surface adsorbed motors or motor fragments (e.g. heavy meromyosin; HMM) propel fluorescence labelled cytoskeletal filaments (e.g. actin filaments). On basis of this assay, methods have been developed to use the filaments as shuttles for cargo attachment and spatially controlled transport on chemically or topographically nanopatterned/micropatterned surfaces. A range of complex devices are now emerging but here we focus on recent advancements towards actomyosin powered biosensing systems. These rely on antibody-attachment to actin filaments using heterobifunctional cross-linkers with consistently maintained HMM driven transportation of the filaments when loaded with antibody-antigen complexes in vitro. Exploiting the fast actomyosin transportation, we show that motor driven filamentguiding along nanosized tracks can achieve rapid (within seconds) enrichment of analytes (antigens) on detector sites. Results relating to approaches for extending device shelf-life and ensuring effective operation in complex fluid environments, e.g. blood serum, will be described.

The advances open for new paradigms in nanoseparation and biosensing with high sensitivity, extreme miniaturization (100 devices $/ \mathrm{mm}^{2}$ ) and extensive multiplexing. Commercial potential is foreseen in medical diagnostics, environmental monitoring etc. but the studies will also give new fundamental insights into actomyosin function not readily obtained in conventional biophysical studies.

\section{5}

Neonatal exposure to chronic intermittent hypoxia causes respiratory muscle weakness in male and female rats

\section{F.B. McDonald ${ }^{1}$, K.D. O’Halloran ${ }^{2}$}

${ }^{1}$ School of Medicine and Medical Science, Health Sciences Centre, University College Dublin, Dublin, Ireland; ${ }^{2}$ Department of Physiology, University College Cork, Cork, Ireland

Chronic intermittent hypoxia $(\mathrm{CIH})$ is a feature of respiratory diseases such as obstructive sleep apnoea and apnoea of prematurity. There is a little information on the effects of $\mathrm{CIH}$ on respiratory muscle physiology despite the clinical relevance. Stimulus pattern, 
intensity and duration are important factors driving phenotypic plasticity in skeletal muscle; moreover age and sex are important factors in determining the capacity of the muscle to re-model. Aim: We sought to investigate the effect of $\mathrm{CIH}$ during early life on the structural and functional properties of respiratory muscles of male and female rats.

Three Wistar litters (with respective dams) were exposed in commercial environmental chambers to intermittent hypoxia (21-5\% $\mathrm{O}_{2} ; 12$ cycles per hour; $8 \mathrm{~h}$ /day for 21 consecutive days) from the first day of life (P0). Sham experiments (continuous normoxia) were run in parallel. After chronic gas treatments, diaphragm and sternohyoid (accessory muscle of breathing) tissue was harvested for structural, functional, and biochemical assessment. Contractile and endurance properties of respiratory muscle was determined in an in vitro isometric bath preparation in Krebs solution bubbled with $95 \% \mathrm{O}_{2} / 5 \%$ $\mathrm{CO}_{2}$ at $35^{\circ} \mathrm{C}$.

$\mathrm{CIH}$ caused a significant decrease in sternohyoid force-frequency relationship in male and female rats $(P<0.05 ; 2$-way ANOVA). Peak sternohyoid muscle force was significantly decreased in male $(14.1 \pm 0.7$ vs. $11.5 \pm 1.0$; mean $\pm \mathrm{SE}$; sham vs. $\mathrm{CIH})$ and female $(16.2 \pm 1.0$ vs. $\mathrm{CIH} 11.5 \pm 0.4) ; \mathrm{N}=7-8$ for all groups.

$\mathrm{CIH}$ exposure in early life causes airway dilator muscle weakness which in human infants could increase the propensity for airway narrowing or collapse.

\section{1}

Late sodium current inhibition reverses electro-mechanical dysfunction in human hypertrophic cardiomyopathy

R. Coppini ${ }^{1,3}$, C. Ferrantini ${ }^{2,3}$, L. Yao ${ }^{4}$, B. Tosi ${ }^{2,3}$, S. Suffredini ${ }^{1,3}$, C. Tesi ${ }^{2,3}$, I. Olivotto ${ }^{5}$, L. Belardinelli ${ }^{4}$, E. Cerbai ${ }^{1,3}$, A. Mugelli ${ }^{1,3}$, C. Poggesi $\mathrm{s}^{2,3}$

${ }^{1}$ Department of Preclinical and Clinical Pharmacology, University of Florence, Italy; ${ }^{2}$ Department of Physiology, University of Florence, Italy; ${ }^{3}$ Center of Molecular Medicine (C.I.M.M.B.A.), University of Florence, Italy; ${ }^{4}$ Gilead Sciences Inc., Palo Alto, USA; ${ }^{5}$ Referral Center for Cardiomyopathies, Careggi University Hospital, Florence, Italy

Hypertrophic cardiomyopathy (HCM), the most common Mendelian heart disorder, remains a therapeutically orphan disease because of the limited understanding of cellular mechanisms underlying arrhythmogenicity and diastolic dysfunction. We assessed the electromechanical profile of cardiomyocytes from $26 \mathrm{HCM}$ patients undergoing myectomy, compared with non-failing non-hypertrophic surgical patients. In patch-clamp and intracellular $\mathrm{Ca}^{2+}\left(\mathrm{Ca}_{\mathrm{i}}^{2+}\right)$ studies, HCM cardiomyocytes showed: (1) prolonged action potential related to decreased repolarizing $\mathrm{K}^{+}$currents and increased late $\mathrm{Na}^{+}\left(\mathrm{I}_{\mathrm{NaL}}\right)$ and $\mathrm{Ca}^{2+}$ currents, (2)increased cellular arrhythmias, (3)prolonged $\mathrm{Ca}^{2+}$ transients, and (4)higher diastolic $\mathrm{Ca}_{\mathrm{i}}^{2+}$. Such changes were related to enhanced $\mathrm{Ca}_{\mathrm{i}}^{2+} /$ calmodulin kinase II (CaMKII) activity and the increased phosphorylation of its targets. Ranolazine, at therapeutic concentrations, reversed the HCM-related cellular abnormalities via selective $\mathrm{I}_{\mathrm{NaL}}$ inhibition, with negligible effects in controls. By shortening action potential duration in HCM cardiomyocytes, ranolazine reduced the occurrence of cellular arrhythmias, such as early after depolarizations. Additionally, owing to the faster kinetics of $\mathrm{Ca}_{\mathrm{i}}^{2+}$ transients and the lower diastolic $\mathrm{Ca}_{\mathrm{i}}^{2+}$, ranolazine improved the diastolic function of $\mathrm{HCM}$ myocardium, evaluated in isometrically contracting trabeculae. Our observations highlight a specific set of functional changes in HCM myocardium, which stem from a complex remodeling process involving alterations of CaMKII-dependent signaling, rather than being a direct consequence of causing sarcomeric mutations. Among the several changes identified, increased $\mathrm{I}_{\mathrm{NaL}}$, leading to altered $\mathrm{Na}^{+}$and $\mathrm{Ca}^{2+}$ homeostasis, appears to play a critical role in the cellular pathophysiology of HCM. Therefore $\mathrm{I}_{\mathrm{NaL}}$ inhibition may have an important therapeutic role for the management of arrhythmias and diastolic dysfunction in HCM patients.

\section{4}

\section{Preclinical experience and perspectives of a clinical trial using} CD133 stem cells

M. Meregalli ${ }^{1}$, A. Farini ${ }^{1}$, M. Belicchi ${ }^{1}$, D. Parolini ${ }^{1}$, C. Sitzia ${ }^{1}$, P. Razini ${ }^{1}$, G. Del Fraro ${ }^{1}$, L. Cassinelli ${ }^{1}$, V. Angeloni, J.C. da Silva

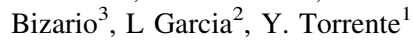

${ }^{1}$ Università degli Studi di Milano, Fondazione IRCCS Cà Granda Ospedale Maggiore Policlinico di Milano, Centro Dino Ferrari, Department of Neurological Sciences, Stem Cell Laboratory, Milan, Italy; ${ }^{2}$ UMR S 787, INSERM/UPMC, Institut de Myologie, Facultè de Medecine Pierre et Marie Curie, Paris Cedex 13, France; ${ }^{3}$ AADM/ UNAERP Ribeirao Preto-SP-Brazil

Cell therapy is one promising approach to correct genetic diseases by contributing to tissue regeneration; stem cells can be isolated from a healthy donor or, when possible from the same patient. In the first case cells will be transplanted under a regime of immune suppression while in the second case, cells will have to be genetically corrected before transplantation in the same patient.

We isolated CD133+ cells from muscle biopsies of GRMD dogs. Dogs one year old, were treated with their own trasnduced U7exon 6-8 CD133+ cells. Dogs received 2 arterial systemic injections through a catheter that was introduced in the left femoralis artery and reached the aortic arch at the level of the left subclavia.

After the injection, all treated dogs showed clinical improvement (including functional performance, in tests such as time to climb stairs, swimming, 6 min walking test). All animals were analysed at different times; most of the biopsies in all muscles had a morphological amelioration when compared to untreated dogs. Dystrophin expression in the biopsies was variable, ranging from 2 to $\geq 7 \%$ in several biopsies of the injected legs. Western blot analysis of different biopsies of the same muscles confirmed the presence of different amount of dystrophin, varying from an undetectable signal to around $6 \%$.

Because of these results, we plan a pilot clinical trial, based on intra-muscular and intra-arterial transplantation of autologous engineered muscle derived CD133+ cells. Efficacy and possible adverse effects will be evaluated to test whether this approach may represent a first step towards an efficacious therapy for muscular dystrophy.

\section{4}

\section{Contractile properties of myometrium in twin pregnancies}

P. Turton, S. Arrowsmith, J. Prescott, C. Ballard, J. Neilson, S. Wray

Department of Cellular and Molecular Physiology, and *Women \& Child Health University of Liverpool

Around $50 \%$ of twin pregnancies end in a preterm labour. To investigate the reasons for this, we examined contractile properties and responses to agonists and relaxants, of myometrium from twin pregnancies and the effect of increasing neonatal birth weight.

Myometrial biopsies from consenting women undergoing elective Caesarean section were dissected into longitudinal muscle strips, superfused with physiological saline solution, and spontaneous contractile activity measured. In some experiments strips were depolarised with a high potassium solution, to compare contraction 
amplitude against amplitude when depolarised. In other experiments oxytocin was applied and the increase in amplitude between twins and singleton pregnancies compared. In a third set of experiments, increasing doses of the relaxant, progesterone were compared.

Myometrium from twin pregnancies $(n=47)$ had contractions that were significantly shorter, more frequent, and an amplitude that was significantly increased with oxytocin, than myometrium from singleton pregnancies. These differences only occurred at term. In all samples, duration significantly decreased with increasing neonatal birth weight, whilst frequency and response to oxytocin increased significantly.

Progesterone decreased myometrial activity in both singletons and twins, but to a lesser extent in twin pregnancies, suggesting that they are more resistant to its effects, consistent with clinical findings.

We have shown that there are differences in contractility and responses to agonist and tocolytics, between singleton and twin pregnancies.

\section{1}

In vivo functional, anatomical and metabolic investigations of a nemaline myopathy mouse model carrying TPM3 mutation

C. Gineste, Y. Le Fur, C. Vilmen, E. Pecchi, P.J. Cozzone, D. Bendahan, J. Gondin

CRMBM CNRS UMR 7339, Aix-Marseille Univ, Faculté de Médecine La Timone, Marseille, France

Nemaline myopathy (NM) is a genetically and clinically highly heterogeneous congenital myopathy and is characterized by muscle weakness and the presence of rod shaped structures in skeletal muscle fibres. Met9Arg mutation in the TPM3 gene causes mild form of NM in humans and is associated with a late-onset of muscle weakness ${ }^{1}$. In the present study, we aimed at reporting the first longitudinal followup of the TPM 3 mouse model and at providing unique metabolic, anatomic and functional information related to this mutation.

In vivo investigations of gastrocnemius muscles were performed in a group of transgenic (Tg) and wild type (WT) mice at 3, 5 and 8 monthold using an experimental set-up ${ }^{2}$ which allows non-invasive measurements of hindlimb muscle volume, force production at various stimulation frequencies, and energy metabolism using ${ }^{31} \mathrm{P}$-magnetic resonance spectroscopy.

$\mathrm{Tg}$ mice were stronger than WT littermates at 5 and 8 months (Fig. 1a). Force frequency curve was shifted to the right in Tg mice at 3 months (Fig. 1b). Muscle volume and metabolic variations were similar between the two groups at all ages. Surprisingly and on the contrary to what has been previously reported ${ }^{1}$, we demonstrated an improved maximal force in $\mathrm{Tg}$ mice. These findings might be explained by an alteration of calcium homeostasis and/or of cross-bridge cycling kinetics. Overall, the TPM3 mouse model does not reproduce the phenotype observed in patients carrying this
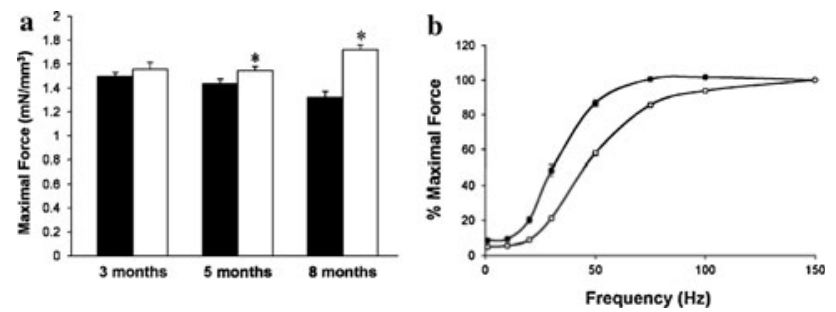

Fig. 1 Data obtained in wild type (black) and transgenic (white) mice. a Maximal force, normalized to muscle volume. b Force frequency curve obtained at 3 months old. Force is normalized to maximal force mutation. Further studies are warranted in order to determine the underlying cellular/molecular mechanisms accounting for the increased contractile function.

\section{References}

[1] Corbett et al. Hum Mol Genet: 317-328, 2001.

[2] Giannesini et al. Magn Reson Med: 262-270, 2010.

\section{2}

In vivo functional, anatomical and metabolic investigations of the ACTA1 (H40Y) mouse model of severe nemaline myopathy

C. Gineste, Y. Le Fur, C. Vilmen, E. Pecchi, P.J. Cozzone,

D. Bendahan, J. Gondin

CRMBM CNRS UMR 7339 Faculté de Médecine La Timone, Marseille, France

Nemaline myopathy (NM) is the most common of the non-dystrophic congenital myopathies and is characterized by muscle weakness. Mutations in the $\alpha$-skeletal actin gene have been found in up to $50 \%$ of the most severely affected cases. Interestingly, a mouse model ${ }^{1}$ carrying the human $\mathrm{H} 40 \mathrm{Y}$ mutation displays all the clinical and pathological characteristics of NM patients. In the present study, we aimed at reporting the first in vivo anatomical, functional and metabolic characterization of the $\mathrm{H} 40 \mathrm{Y}$ mouse model. In vivo investigations of gastrocnemius muscles were performed in 14 weekold H40Y and wild type (WT) female mice using an experimental set$u^{2}$ which allows non-invasive measurements of hindlimb muscle volume, force production at various stimulation frequencies, and energy metabolism using ${ }^{31} \mathrm{P}$-magnetic resonance spectroscopy. Muscle volume was $17 \%$ smaller in H40Y mice. Normalized maximal force was $35 \%$ lower in H40Y mice as compared to WT (Fig. 1a). Fatigability was reduced in H40Y mice as compared to WT (Fig. 1b). Metabolic changes were similar between the two groups.

The large reduction of in vivo maximal force production is in agreement with what has been reported in vitro in isolated $\mathrm{H} 40 \mathrm{Y}$ muscles while the reduced fatigability might be explained by the shift toward a slower phenotype recently observed in this model ${ }^{1}$. On the contrary to what has been previously suggested ${ }^{3}$, severe forms of NM are not necessarily associated with an impaired energy metabolism. Further studies are warranted in order to unravel the underlying cellular/molecular mechanisms accounting for the decreased contractile function.

\section{References}

[1] Nguyen et al. Brain:3516-3529, 2011.

[2] Giannesini et al. Magn Reson Med:262-270, 2010.

[3] Sanoudou et al. Hum Mol Genet:2603-2612, 2006.
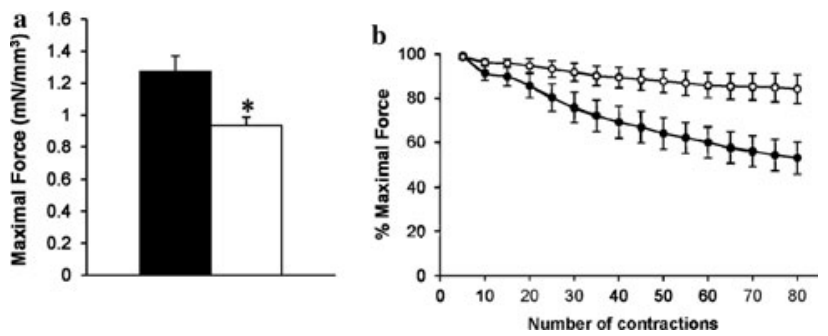

Fig. 1 Data obtained in wild type (black) and H40Y (white) mice. a Maximal force, normalized to muscle volume. b Force obtained during 80 contractions at $40 \mathrm{~Hz}$. Force is normalized to maximal force 


\section{3}

\section{Lactate significantly decreases in vitro myometrial contractility}

J.A. Hanley ${ }^{1}$, A. Weeks ${ }^{2}$, S. Wray ${ }^{1}$

${ }^{1}$ University of Liverpool, Department of Cellular and Molecular Physiology; ${ }^{2}$ Department of Women's and Children's Health, Liverpool, United Kingdom

Weak, uncoordinated uterine contractions (labour dystocia) accounts for $20 \%$ of caesarean sections (CS) in the UK. Lactate is increased in myometrial capillary blood and amniotic fluid of women with labour dystocia, suggesting that lactate may be causing weak contractions, There are however no functional data on its effects on the myometrium.

Myometrial strips from term pregnant rats or biopsies obtained with consent from women undergoing CS were studied. The effects on spontaneous and oxytocin-stimulated contractility in response to sodium lactate $(1-20 \mathrm{mM})$ and another weak acid sodium propionate were investigated. In some experiments, tissue was loaded with Indo$1 \mathrm{AM}$ to simultaneously measure force and intracellular $\mathrm{Ca}^{2+}$ signalling. Lactate significantly decreased spontaneous contractility. A dose dependent decrease in the integral of force (AUC) was seen, which was significant at $5 \mathrm{mM}$ concentration (rat $=36 \% \pm 14 \%$, human $=44 \% \pm 17 \% P<0.05)$ and above. The same was seen with propionate. Lactate's effects were reduced in the presence of oxytocin; $5 \mathrm{mM}$ lactate reduced AUC (rat $=59 \% \pm 15 \%$, human $=64 \% \pm 15 \%, P<0.05)$. $\mathrm{Ca}^{2+}$ data mirrored force. Lactate decreases spontaneous and also, but to a lesser extent oxytocinstimulated contractility. Other weak acids produce similar effects to lactate suggesting its mechanism of action is not via metabolism.

Lactate inhibited $\mathrm{Ca}^{2+}$ transients, which could be due to a fall of intracellular $\mathrm{pH}$ depressing inhibiting L-type $\mathrm{Ca}^{2+}$ channel entry.

Hanley is a candidate for a young investigator award to be judged during the EMC 2012.

\section{4}

\section{The effect of aging on myometrial function in vitro}

\section{S. Arrowsmith, H. Robinson, K. Noble, S. Wray}

University of Liverpool, Department of Cellular and Molecular Physiology, Liverpool, UK

Much is known about the effects of aging in skeletal muscle. However, relatively little is known about aging in smooth muscle. The aim of this study was to examine whether aging affects uterine function, with a focus on myometrial function.

Isometric tension recordings were performed on strips of myometrium $(1 \times 5 \times 2 \mathrm{~mm})$ obtained from pregnant $(\mathrm{PR}, n=80)$ or non-pregnant women (NP, $n=24)$ aged 25-72 years, undergoing caesarean section or hysterectomy respectively. Mean amplitude and integral of force of spontaneous contractions $(20 \mathrm{~min})$ and under high $\mathrm{K}^{+}$stimulation $(2 \mathrm{~min}$ ) were calculated and compared according to maternal age. There was a significant negative correlation between force amplitude and maternal age in NP uterus $(r=-0.529$, $P=0.008)$, however no significant correlation was observed in the $\mathrm{PR}$ uterus. Force normalised to high $\mathrm{K}^{+}$in NP women also declined with age but not in PR women. Aging decreases uterine contractility but only to a significant extent in NP state. Reduced high $\mathrm{K}^{+}$contraction suggests loss of functional muscular tissue or decreased expression of L-type Ca channels with age, possibly attributed to decreased oestrogen levels leading to muscle atrophy.

The lack of effect in PR uterus suggests myocytes respond to the hormonal environment of pregnancy by hypertrophy and hyperplasia, thus restoring any age related deficits from the NP state.

\section{7}

\section{Regenerative potential of a collagen matrix is heightened} in necrotic contexts

D. Kuraitis ${ }^{1,2,3}$, A. Musarò ${ }^{3 *}$, E.J. Suuronen ${ }^{1,2} *$

${ }^{1}$ University of Ottawa Heart Institute, Division of Cardiac Surgery, Ottawa, Canada; ${ }^{2}$ University of Ottawa, Department of Cellular \& Molecular Medicine, Ottawa, Canada; ${ }^{3}$ University of Rome La Sapienza, DAHFMO-Unit of Histology and Medical Embryology, Rome, Italy; *Equal contribution

Previous studies have shown that an injectable, collagen-based matrix can stimulate regeneration of ischemic muscle. This study sought to characterize its potential for treatment of myopathies and investigate the role of necrosis in matrix-augmented regeneration.

EDL muscles of mdx mice and MLC/SOD ${ }^{\mathrm{G} 93 \mathrm{~A}}$ received injections of PBS or matrix $(n=5)$. Treated muscles were analyzed using treadmill and qPCR. Satellite cell cultures from C57BL/6 mice were maintained under standard conditions or on the matrix \pm the addition of necrotic myocyte debris (NMD), prepared from dead myocytes. After $24 \mathrm{~h}$, myotubes were analyzed $(n=5)$ and conditioned medium $(\mathrm{CM})$ was collected, applied to $\mathrm{C} 2 \mathrm{C} 12$ myoblast cultures $(n=3)$ and screened using cytokine arrays $(n=6)$.

Matrix-treated mdx mice ran $41 \%$ further and $22 \%$ faster and displayed increased Pax3, myogenin, desmin \& Myf5 transcript levels $(P<0.04)$. No differences were observed in treated MLC/ $\mathrm{SOD}^{\mathrm{G} 93 \mathrm{~A}}$ animals. Matrix exposure increased myotube numbers by 3.3-fold and MyoD, myogenin \& Mef2C transcript levels $\leq 50 \%$. Matrix exposure +NMD greatly increased these transcripts $\leq$ $1210 \%(P<0.05)$. Application of CM from matrix-NMD cultures accelerated Myf5, myogenin and Mef2C transcription in $\mathrm{C} 2 \mathrm{C} 12$ myoblasts $(P<0.01)$. This CM contained elevated levels of HGF and SDF-1 $(P<0.04)$.

In vitro observations suggest that matrix-augmented myogenesis is amplified in necrotic environments. In vivo, matrix-induced myogenesis was observed in the constant necrosis $m d x$ animal model, but not in the atrophic model. Furthermore, satellite cell-matrix interactions in a necrotic context increase the secretion of regenerative cytokines, leading to more rapid myoblast maturation via paracrine effects.

\section{5}

Voltage-gated ion channel impairments in the dystrophic heart

X. Koenig ${ }^{1}$, L. Rubi ${ }^{1}$, S. Dysek ${ }^{2}$, S. Kummer ${ }^{2}$, A.K. Mike ${ }^{1}$, V. Gawali ${ }^{1}$, P. Lukacs ${ }^{1}$, H. Todt ${ }^{1}$, R.E. Bittner ${ }^{2}$, K. Hilber ${ }^{1}$

Medical University of Vienna, Center for Physiology and Pharmacology, Vienna, Austria; ${ }^{2}$ Medical University of Vienna, Center for Anatomy and Cell Biology, Vienna, Austria

Duchenne muscular dystrophy (DMD) is an inherited disease characterized by progressive muscle weakness and degeneration. Besides the relatively well characterised skeletal muscle degenerative processes, DMD is also associated with severe cardiovascular complications including cardiomyopathy and cardiac arrhythmias. The current understanding of the patho-mechanisms is still very limited, but recent research suggests that dysfunctional ion channels in dystrophic cardiomyocytes considerably contribute to the cardiovascular complications.

By using the whole cell patch clamp technique, currents through voltage-gated $\mathrm{Na}$ and $\mathrm{Ca}$ channels were recorded in ventricular cardiomyocytes isolated from the hearts of adult normal and dystrophic mice. Besides the commonly used dystrophin-deficient mdx mouse model for human DMD, we also used mdx-utr mice, which are both dystrophin- and utrophin-deficient. 
We found that Na currents were dramatically reduced in dystrophic cardiomyocytes, whereby extra utrophin-deficiency generated a stronger current reduction than dystrophin-deficiency alone. In addition, $\mathrm{Ca}$ currents were significantly increased, and $\mathrm{Ca}$ channel inactivation was reduced in dystrophic cardiomyocytes. These ion channel impairments in cardiomyocytes derived from dystrophic mice go along with altered surface electrocardiogram parameters in these animals.

Voltage-gated ion channels are significantly impaired in dystrophic cardiomyocytes. These impairments very likely perturb electrical impulse propagation in the dystrophic heart, and thus contribute to the cardiac complications associated with the muscular dystrophies.

Supported by the Austrian Science Fund FWF (P19352 and P23060).

\section{0}

\section{Effect of S107 on human primary and fibroblast-derived myotubes}

I. Toral $^{1,2}$, G. Aldanondo ${ }^{1}$, P. Ferrón ${ }^{3}$, J.I. Miranda ${ }^{3}$, J.M. Aizpurua ${ }^{3}$, A. López de Munain ${ }^{1,2}$, A. Vallejo-Illarramendi ${ }^{1,2}$

${ }^{1}$ Instituto de Investigación Biodonostia, Neurociencias, San Sebastián, Spain; ${ }^{2}$ CIBERNED, Centro de Investigaciones Biomédicas en Red sobre Enfermedades Neurodegenerativas, Instituto Carlos III, Spain; ${ }^{3}$ Universidad del País Vasco, Facultad de Químicas, San Sebastián, Spain

Muscular dystrophies are characterized by primary wasting of skeletal muscle, which compromises patient mobility and can lead to complete paralysis and premature death. Impairment of calcium transport is one contributing factor to the clinical phenotype of Duchenne muscular dystrophy. A recent study in mdx mice, model of this dystrophy, describes abnormal nitrosylation of ryanodine receptor 1 (RyR1) that causes depletion of calstabin-1 from the RyR1 complex and results in 'leaky' channels. Treatment of these mice with the RyR1 stabilizer S107, prevents calstabin-1 depletion, inhibits sarcoplasmic reticulum calcium leak and reduces biochemical and histological evidence of muscle damage while improving muscle function and exercise performance. However, up to date, S107 has not been tested on human samples in vitro.

In this study, we have analyzed the effect of S107 on human primary and fibroblast-derived myotubes. We found that S107 concentrations up to $100 \mathrm{uM}$ are non-toxic to these cells, although treatment with $1 \mathrm{mM} \mathrm{S107}$ for $24 \mathrm{~h}$ resulted in $100 \%$ myotube death. Next, we used Fluo4-AM fluorochrome to measure the changes of free intracellular calcium with $\mathrm{KCl}$ and caffein. We found that addition of S107 to these cells results in significant decreases in calcium responses. Also, in situ proximity assay analysis shows increase colocalization of RyR1 and calstabin-1 in cells treated with S107.

Our results obtained in human myotubes are in line with the ones obtained in mouse treated with S107, and suggest that this drug might be an appropriate candidate for a clinical trial with Duchenne patients.

\section{2}

Effects of male sex steroids in isolated intact mammalian skeletal muscle fibre bundles: implications for the management of sarcopenia

M.M. Hamdi, G. Mutungi

Department of Medicine, Norwich Medical School, University of East Anglia, Norwich NR4 7TJ, United Kingdom
Sex steroids are important determinants of body structure, composition and function. For example, treating adult female laboratory animals, hypogonadal men and elderly men with low testosterone (T) concentrations with $\mathrm{T}$ or any of its many derivatives for several weeks will increase lean body mass. These findings suggest that male sex hormones are important in the maintenance of skeletal mass and that they may be useful in the management of sarcopenia. Also, little is known about their acute effects in mammalian skeletal muscle fibres.

In this study, we investigated the effects of treating small skeletal muscle fibre bundles, isolated from the extensor digitorum longus (edl, a fast-twitch muscle) and the soleus (a slow-twitch muscle) of adult mice, with physiological concentrations of $\mathrm{T}$ and dihydrotesterone (DHT) for $1 \mathrm{~h}$. Our results show that treating the fibre bundles with DHT, but not $\mathrm{T}$, increases force production and amino acid uptake in the fast-twitch fibres only. It also increases the phosphorylation of ERK1/2, RSK1/2 and that of the 20KDa regulatory myosin light chains. Moreover, all the effects are abolished by the MEK inhibitor PD98059; this suggests that they are mediated through the MAPK pathway. DHT treatment also increased the expression of the L-type amino acid transporter (LAT) 2 in the fasttwitch fibres. It is important to note that fast-twitch fibres are the ones lost during aging.

From these findings we suggest that DHT is the more potent hormone in adult mammalian skeletal muscles and that it may have beneficial effects in sarcopenia.

\section{6}

Control of transport in myosin-based nanodevices by divalent cations

F.K. Hartmann, N. Hundt, R.P. Diensthuber, I. Chizhov, G. Tsiavaliaris

Institute for Biophysical Chemistry, OE 4350, Hannover Medical School, Feodor-Lynen-Str. 5, 30625 Hannover, Germany

Processive myosin-5 molecules have the potential to be used as molecular nanomachines for transportation of cargo in biohybrid devices. Feasibility of this implementation is favored by previous studies, where we engineered single- and double headed myosin-5 motors and characterized their mechanochemical properties. An important outcome of our investigations was the observation that velocity of actin filament sliding induced by surface immobilized myosin-5 molecules can be easily controlled by free $\mathrm{Mg}^{2+}$-ions. This approach offers the opportunity to regulate transport processes in a defined manner.In order to understand the mechanism of regulation, we analyzed $\mathrm{Mg}^{2+}$-concentration and temperature dependences of myosin-5 motility using wild-type and $\mathrm{Mg}^{2+}$-insensitive motor domain constructs. Our experiments revealed a strong non-linear dependence of the logarithm of sliding velocity on the reciprocal temperature. This behavior can be explained by the introduction of a fast equilibrium on the reaction pathway, which precedes the force generating state of myosin-5 enzymatic cycle. We found that this equilibrium is both temperature and $\mathrm{Mg}^{2+}$-dependent and provides an appropriate description of the convex Arrhenius behavior. Data analysis shows that the $\mathrm{Mg}^{2+}$-bound state is entropically favorable. This is in line with other experiments showing that divalent cations have higher entropy in bound form for many protein and organic compounds.

The present work describes a thermodynamic approach as essential prerequisite for understanding regulatory mechanisms of myosins and is important in the future design of biohybrid-based nanodevices to achieve spatiotemporal control over a range of transport function. 


\section{8}

Early stages of knee osteoarthritis alter type $2 \mathrm{~b}$ fibers of the vastus lateralis muscle and decrease eccentric knee extensor torque

P.R. Serrão ${ }^{1}$, F.A. Vasilceac ${ }^{1}$, A.C. Mattiello-Sverzut ${ }^{2}$, S.M. Mattiello ${ }^{1}$

${ }^{1}$ Federal University of São Carlos, Department of Physical Therapy, São Carlos, Brazill; ${ }^{2}$ Sao Paulo University, Department of Biomechanics, Medicine and Rehabilitation of the Locomotor Apparatus, Ribeirão Preto, Brazil

The quadriceps muscle weakness has been identified as a potential risk factor in the progression of osteoarthritis. Therefore, it is necessary to conduct morphological and functional studies in the early stages of the disease. The purpose of this study was to evaluate the morphological alterations of the vastus lateralis muscle (VL) and knee extensor torque (KET) of the subjects with early stages of knee osteoarthritis.Forty men participated in this study: control group (CG; $n=18$; healthy subjects; $51.8 \pm 6.4$ years) and osteoarthritis group (OAG; $n=22$; subjects with grade I or II knee osteoarthritis; $52 \pm 8.1$ years). The biopsy of the VL was performed for morphometric analysis. Biopsy specimens were taken using the percutaneous needle biopsy method of Bergström. Muscle sections were stained to measure myosin adenosine triphosphatase (mATPase). The assessment of KET was performed using an isokinetic dynamometer (Biodex System 3, Biodex Medical Inc.). Concentric and eccentric KET were evaluated $\left(90^{\circ} \mathrm{s}\right.$ and $180 \%$ s). The data were analyzed using Statistica ${ }^{\circledR}$ software (7.0, StatSoft Inc.). $T$ test for independent samples and the Mann-Whitney $U$ test $(\alpha<0.05)$ were used. Significantly higher values of the fiber number percentage of type $2 b$ fibers $(P=0.02)$ were found in the OAG. Significant differences were found between the groups in eccentric KET at $90 \% \mathrm{~s}(P=0.01)$ and at $180^{\circ} \mathrm{s}(P=0.04)$ with higher values for the CG.

In conclusion, men with early stages of knee OA already have concomitant increases in the proportion of type $2 b$ fibers as well deficits in KET, which may indicate possible mechanisms of neuromuscular adaptation.

\section{1}

Effect of human tendon conditioning on muscle structure and contractile force-generating ability

\section{C.N. Maganaris}

Institute for Biomedical Research into Human Movement and Health (IRM), Manchester Metropolitan University, Manchester M1 5GD, UK

Tendons are not perfectly elastic structures, but exhibit a timedependent mechanical behaviour when stretched. In isolated-tendon tests, this viscoelasticity can be documented in several ways, one of which is the development of creep, i.e., an increasing tendon deformation with time for a given tensile load. Increasing deformations for a given load, however, can also result from inadequate fixation of some collagen fibres in the tendon by the clamps used, which has often been assumed to be the case in the initial test loading cycles, a phenomenon commonly referred to as "conditioning". If, however, this behaviour could also be observed in vivo, then conditioning would be an actual property - not a measurement artefact, and this would have consequences for the structure and function of the inseries muscle.

Experiments on in vivo human gastrocnemius tendons stretched by the contractile force generated by isometric plantarflexion contraction show that, indeed, the tendons experience creep in the first few contraction/relaxation cycles. This makes the gastrocnemius muscle shorten its fibres and increase its pennation more in the transient conditioning phase, before a stable response is observed. Calculations based on the cross-bridge theory of contraction indicate that the creep-induced muscle shortening would shift the average sarcomere in the gastrocnemius muscle towards less favourable operating lengths for force generation, with the contractile force transmitted to the tendon being further reduced due the larger pennation. In addition, the extra muscle shortening induced by creep could be seen by the muscle spindles. The former could be mistaken as evidence for neuromuscular fatigue, and the latter could complicate joint positional control.

\section{3}

Muscle size and metabolism in end stage renal failure patients G.K. Sakkas ${ }^{1}$, C. Karatzaferi ${ }^{2}$

${ }^{1}$ Centre for Research and Technology, Thessaly, Muscle Function \& Clinical Studies Laboratory, Trikala, GR; ${ }^{2}$ University of Thessaly, DPESS, Muscle Physiology \& Mechanics Group, Trikala, GR

Hemodialysis patients are characterized by progressively worsening muscle weakness and muscle atrophy due to both a metabolic and a disuse component collectively described as uremic myopathy. Various interventions in stable dialysis patients while have helped these patients improve their functionality, still have not proven enough to bring their muscle quality and quantity up to the levels of a healthy sedentary person. Moreover, patients present with sleep problems, anxiety and or symptoms of depression but most notably they complain of chronic fatigue and weakness.

Hemodialysis patients have 10-20 times a greater risk of cardiac death than healthy individuals, despite stratification for the traditional risk factors such as age, race, sex and diabetes. Insulin resistance significantly contributes to the increased cardiovascular morbidity and mortality in these patients while it is present in almost all patients with end-stage renal disease. Fatty liver is another confounding factor affecting whole body metabolism and reducing muscle anabolism. Skeletal muscles are not only the locomotory machinery of our body but have been shown to act as an endocrine organ producing and releasing myokines, which work in a hormone-like fashion, exerting specific endocrine effects on other organs such as the liver and the adipose tissue.

The low muscle mass seen in HD patients is probably one of the main causes of the metabolic perturbations often observed in these patients which account for their high mortality rate. Exercise interventions have helped improve both muscle quantity and quality, and lowered overall risk, with benefits also evident in patient perceptions of quality of life.

\section{3}

The pro-forms of insulin-like growth factor I (IGF-I) are predominant in skeletal muscle and alter IGF-I receptor activation

A. Philippou, J. Durzyńska, M. Nguyen-McCarty, B.K. Brisson, E.R. Barton

University of Pennsylvania, Department of Anatomy and Cell Biology, School of Dental Medicine, and Pennsylvania Muscle Institute, Philadelphia, PA, USA

Insulin-like growth factor I (IGF-I) is a key regulator of muscle development and growth. The pre-pro-peptide produced by the IgfI gene undergoes several post-translational processing steps to result in 
a secreted mature protein, which is considered to be the obligate ligand for the IGF-I receptor. The goals of this study were to determine what forms of IGF-I exist in skeletal muscle, and if the mature IGF-I protein was the only form able to activate the IGF-I receptor.

We utilized viral mediated gene transfer to produce high levels of IGF-I in muscle, and also isolated protein from control muscle. Forms of this protein were assessed by immunoblotting and ELISA, and post-translational modifications were tested by furin and glycosidase incubation. To determine if the all IGF-I forms were ligands for the IGF-I receptor, we generated each specific form through transient transfection of 3T3 cells, and utilized the enriched media to perform a kinase receptor (KIRA) assay.We found that the predominant forms were pro-IGF-I and glycosylated pro-IGF-I, which retained the C-terminal E peptide extension, in lieu of mature IGF-I. These forms were validated using samples subjected to viral expression of IGF-I combined with furin and glycosidase digestion. Compared to mature IGF-I, pro-IGF-I was almost 2-fold more efficient at IGF-I receptor phosphorylation, whereas glycosylation of the pro-IGF-I significantly reduced receptor activation.

It is important to understand not only the quantity, but also the proportion of IGF-I forms produced, to evaluate the true biological activity of this growth factor, because not all forms provide equivalent activity. Further, development of different forms of IGF-I may have greater therapeutic benefit for muscle than mature IGF-I.

Supported by NIH AR057363.

\section{5}

\section{Functional characterisation of a titin kinase mutation} in a novel myopathy

A.L. Kho ${ }^{1}$, C. Chauveau ${ }^{2,5}$, C. Bonnemann ${ }^{6}$, A. Alexandrovich ${ }^{1}$,

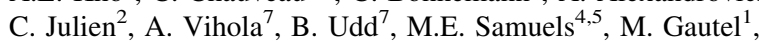
A. Ferreiro ${ }^{2,3}$

${ }^{1}$ King's College London, Cardiovascular Division \& Randall Division for Cell and Molecular Biophysics, London, UK; ${ }^{2}$ INSERM/UPMC, UMR787 Groupe myologie, GH Pitié-Salpêtrière, Paris, France; ${ }^{3}$ Centre de Référence des maladies neuromusculaires, GH PitiéSalpêtrière, Paris, France; ${ }^{4}$ Dalhousie University, Department of Pathology, Halifax, Nova Scotia, Canada; ${ }^{5}$ Université de Montréal,
Centre de Recherche de l'Hôpital Ste-Justine, Montréal, Canada; ${ }^{6}$ National Institutes of Health, Bethesda, MD, USA; ${ }^{7}$ University of Helsinki, Folkhälsan Institute of Genetics and Department of Medical Genetics, Haartman Institute, Helsinki, Finland

The giant sarcomeric protein, titin, has an essential role as a molecular ruler determining sarcomere length. Furthermore, titin contains a C-terminal kinase domain (TK) located in the M band. TK interacts with the autophagy cargo adaptor protein, nbr1.

A paediatric patient suffering from arthrogryposis and ventricular non-compaction was found to have compound heterozygous mutations in titin. A TK missense mutation targets a residue highly conserved in the CaMK-like kinase family and is also carried by the healthy father. A second heterozygous TTN mutation in intron 38 is also carried by the healthy mother, and results in a premature stop codon. Therefore, the only full-length titin expressed in this patient would be from the missense mutant allele, and is the likely cause of the myopathy.

Yeast two hybrid assays demonstrated that the new TK mutation, W260R, abrogated nbrl binding completely. The mutant kinase showed significantly reduced thermal stability by circular dichroism, but no aggregation tendency in vitro.

Immunohistochemical analysis of heart samples from the patient revealed streaming of the Z-disk proteins alpha-actinin, ZASP, and myotilin that did not colocalise with p62. Localisation of the autophagy proteins nbr1 and SQSTM1 showed increased nbr1/ SQSTM1-positive puncta (autophagosomes). The ratio of LC3-1 to LC3-2, an indication of autophagic flux, was increased. Protein levels of telethonin, an in vitro substrate of titin kinase, were decreased but localisation to Z-disks seemed unchanged. In contrast, myotilin and ZASP were found to be strongly increased. In summary, a novel recessive loss-of-function mutation in titin kinase causes disrupted protein turnover with signs of defective autophagy, and striking Z-disk alterations reminiscent but not identical to myofibrillar myopathies.

Open Access This article is distributed under the terms of the Creative Commons Attribution License which permits any use, distribution, and reproduction in any medium, provided the original author(s) and the source are credited. 


\section{Author Index}

\begin{tabular}{|c|c|c|c|c|c|}
\hline Aagaard P. & 245 & Brunello E. & 258 & Di Santo J. & 278 \\
\hline Aamiri A. & 278 & Bruusgaard J.C. & 245,275 & Dienes B. & 242 \\
\hline Adamek N. & 236 & Bucci L. & 268 & Diensthuber R.P. & 283 \\
\hline Addario B. & 270 & Buck C. & 242 & Dierck F. & 245 \\
\hline Agatea L. & 240 & Buck D. & 269,265 & Dijk F.J. & 272 \\
\hline Agbulut O. & 276 & Bullard B. & 239 & Dinu G. & 241,249 \\
\hline Ahtiainen M. & 261 & Burdyga $\mathrm{T}$. & 278 & Djinović-Carugo K. & $248,253,255$, \\
\hline Aizenbud D. & 271 & Busolin G. & 260,262 & & 261,270 \\
\hline Aizpurua J.M. & 282 & Butler-Brown G. & 271 & Djinovic-Carugo K. & 248,252 \\
\hline Akimoto $\mathrm{T}$. & 275 & Butler-Browne G. & $272,276,278$ & Doi N. & 251 \\
\hline Albertini C. & 261 & & & Dolfi M. & 257 \\
\hline Albet-Torres N. & 278 & Cabrera-Fuentes H. & 276 & Dollhopf M. & 240 \\
\hline Aldanondo G. & 282 & Cação-Benedini L.O. & 263,264 & Donaldson A. & 274 \\
\hline Alen M. & 261 & Campbell E.L. & 268 & Dorfmüller P. & 269 \\
\hline Alexandrovich A. & 284 & Canato $\mathrm{M}$. & $239,245,259$ & Dorfmuller P. & 269 \\
\hline Ammar T. & 248 & Cancellara P. & $260,262,239,240$ & Dorovkov M.V. & 250 \\
\hline Andersen L.L. & 237 & Canepari M. & 244 & dos Remedios C. & 244,269 \\
\hline Andresen C. & 256 & Cannavino $\mathrm{J}$. & 273 & dos Remedios C.G. & 269 \\
\hline Angelini C. & 275 & Caorsi V. & $256,267,268$ & Durzyńska J. & 284 \\
\hline Angeloni V. & 279 & Capri M. & 261,268 & Dutka T.L. & 244 \\
\hline Antin P.B. & 255 & Caremani M. & 257 & Dux L. & 238,239 \\
\hline Arredondo J.J. & 253,264 & Carlier P.G. & 271 & Dyar K.D. & 240 \\
\hline Arrowsmith S. & 280,281 & Carnio S. & 244,273 & Dysek S. & 282 \\
\hline \multirow[t]{2}{*}{ Azzabou N. } & 271 & Cartoni R. & 275 & & \\
\hline & & Cassinelli L. & 279 & Egawa T. & 275 \\
\hline Baán J. & 238,239 & Cázares-Raga F.E. & 276 & Egner I.M. & 245 \\
\hline Babicz K. & 266 & Cerbai E. & 246,279 & Eldred C. & 239 \\
\hline Babini L. & 261 & Cervera M. & 253,264 & & \\
\hline Backman L. & 270 & Chamberlain J.S. & 277 & Færgemann N. & 238 \\
\hline Ballard C. & 280 & Chatziefthimiou S.D. & 251 & Farini A. & 279 \\
\hline Balogh A. & 242 & Chauveau C. & 284 & Feng H.Z. & 237 \\
\hline Bang M.L. & 259 & Chemello F. & 260,262 & Ferenczi M. & 252 \\
\hline Baraibar M.A. & 272 & Chen L. & 268 & Ferenczi M.A. & $242,256,267,268$ \\
\hline Barbalinardo $\mathrm{M}$. & 248 & Chizhov I. & 283 & Fernández-Valverde $\mathrm{F}$ & 276 \\
\hline Barnouin Y. & 271 & Ciciliot S. & 240 & Fernandez M. & 242 \\
\hline Barton E.R. & 284 & Clima R. & 255 & Ferrón P. & 282 \\
\hline Bean C. & $259,260,262$ & Colson B.A. & 264,266 & Ferrantini $\mathrm{C}$. & 246,279 \\
\hline Beggs A. & 265 & Conte M. & 255,268 & Ferrara C. & 242,246 \\
\hline Beggs A.H. & 269 & Cooke R. & 243 & Ferreiro A. & 284 \\
\hline Belardinelli L. & 279 & Cooney G.J. & 251 & Ferry A. & 276,278 \\
\hline Belicchi M. & 279 & Coppini R. & 246,279 & Fiaschi L. & 248,252 \\
\hline Bellavista E. & 255 & Cornachione A.S. & 267 & Filippin E. & 274 \\
\hline Bendahan D. & 280 & Cozzone P.J. & 280 & Finni $T$. & 239 \\
\hline Bershitsky S.Y. & 242 & Csernoch L. & 242 & Floyd R. & 278 \\
\hline Beuron F. & 251 & Curtin N. & 252 & Fogl C. & 247 \\
\hline Bezerra E. H.S. & 253 & & & Foster D.B. & 243 \\
\hline Bishu K.G. & 257 & Daczewska M. & 261 & Franceschi C. & $255,260,261,268$ \\
\hline Bittner R.E. & 282 & Daggett V. & 236 & Frandsen U. & 237 \\
\hline Blaauw B. & 240,260 & Daly R. & 251 & Frey N. & 245 \\
\hline Bliss K.T. & 250 & da Silva Bizario J.C. & 279 & Friguet B. & 272,273 \\
\hline Bloemink M.J. & 262 & De Almeida Ribeiro E. & 248,261 & Fürst D. & 245,248 \\
\hline Bodmer R. & 264 & D'Errico-Grigioni A. & 255 & Fürst D.O. & 253 \\
\hline Bodnár D. & 242 & de Winter J. & 265 & Fusi L. & 258 \\
\hline Bongardt $\mathrm{S}$. & 266 & de Man F.S. & 269 & & \\
\hline Bonnemann $\mathrm{C}$. & 284 & de Tombe P.P. & 236 & Galler S. & 250 \\
\hline Boontje N. & 244 & De Vito G. & 246 & Garcia L. & 279 \\
\hline Borghetti G. & 261 & Deacon J.C. & 262 & Garófalo M.A. & 274 \\
\hline Borovikov Y.S. & 247 & deAlmeida R.E. & 255,252 & Gautel M. & 253 \\
\hline Bottinelli R. & $244,245,273$ & Degens H. & 246,249 & Gautel M. & $255,265,284$ \\
\hline Brinyiczki K. & 278 & Degiovanni A. & 255 & Gawali V. & 282 \\
\hline Brisson B.K. & 284 & Deineko V. & 248,252 & Geeves M.A. & $236,255,262,266,271$ \\
\hline Brocca L. & 245,273 & Dejong D. & 248 & Geyer N. & 242 \\
\hline Brozovich F.V. & 243 & Del Fraro G. & 279 & Ghisleni A. & 253,255 \\
\hline Brozovich N.J. & 243 & DeWinter J.M. & 269 & Giacomello M. & 259 \\
\hline
\end{tabular}


Gineste C.

Gkougkoulia E.A.

Goldberg A.L.

Gomes A. R.S.

Gondin J.

González-Calixto C.

González-López L.

Goto A.

Goto K.

Graakjær J.

Granzier H.

Gregorio C.C.

Grishkovskaya I.

Guerrero L.

Guignabert C.

Gundersen K.

Gurnett C.A.

Haase $\mathrm{H}$

Hamdani N.

Hamdi M.M.

Handoko M.L.

Hanley J.A.

Hansen E.E.H.

Hartmann F.K.

Hauschka S.D.

Hawes G.P.

Hayward L.

Hegyi G.

Heiny J.

Hernández-Hernández F.

Hespel P.

Hilber K.

Hogrel J.Y.

Holash R.J.

Holt L.J.

Hopkinson N.S.

Hossain M.M. Chen X.

Houdusse A.

Hourd C.é

Hudson P.

Humbert M.

Hundt N.

Hunger M.S.

Hvid L.G.

Hyzewicz J.

Ignatushchenko M.

Ikuta A.

Irving $\mathrm{M}$.

Isabet $\mathrm{T}$.

Jacobsen S.V.

Jagla $\mathrm{K}$.

Jagla $\mathrm{T}$.

Janco M.

Jasper J.R.

Jaspers R.T.

Jenes A.

Jensen L.

Jensen M.M.

Jeong $\mathrm{M}$.

Jiménez Estrada I.

Jin J.P.

Joanne P.
Jones D.A.

Jørgensen L.H.

Julien C.

Just S.

Justyna K.

Kaisari S.

Kalbe C.

Kalganov A.

Kalyva A.

Kaprio J.

Karatzaferi C.

Kashina A.

Kast D.J.

Katzemich A.

Kawai M.

Kaya-Copur A.

Keller-Pintér A.

Kemp P.R.

Képiró M.

Kettelhut I.C.

Kho A.L.

Khogali S.

Kirfel G.

Kitamura F.

Kjaer M.

Knowles A.C.

Kocsis T.

Koenig X.

Köhnke C.

Kolwicz S.C.

Konarev P.

Korte F.S.

Kósa M.

Kostyukova A.S.

Kotlyanskaya L.

Kötter S.

Koubassova N.A.

Koutsilieris M.

Kovács M.

Kovanen V.

Koya $\mathrm{T}$.

Krüger M.

Krieg P.A.

Krishnan R.

Kujala U.

Kumar S.

Kummer S.

Kuraitis D.

Kurosaka S.

Kursula I.

Kursula P.

Labeit S.

Ladouce R.

Lamb G.D.

Lamon S.

Lanfranchi G.

Lang $\mathrm{P}$.

Lanzarini C.

Lard $\mathrm{M}$.

Laveder P.

Lawlor M.

Lazzarini R.

Le Fur Y.

\begin{tabular}{|c|c|c|c|}
\hline 246,249 , & 268 & Léger B. & 275 \\
\hline & 238 & Lehman F. & 257 \\
\hline & 284 & Lehner A. & 261 \\
\hline & 256 & Lehrer S.S. & 246 \\
\hline & 263 & Leinwand L.A. & 262 \\
\hline & & Leonard K. & 239 \\
\hline & 271 & Leu N.A. & 267 \\
\hline & 236 & Leung M.C. & 267 \\
\hline & 267 & Levine B.A. & 250 \\
\hline & 255 & Levitsky D.I. & 247 \\
\hline & 261 & LeWinter J. & 242 \\
\hline 266,275 , & 283 & LeWinter M.M. & 237 \\
\hline & 267 & Lewis A. & 274 \\
\hline & 264 & Li C. & 256 \\
\hline & 239 & Linari $\mathrm{M}$. & 257 \\
\hline & 258 & Lingrel $\mathrm{J}$. & 274 \\
\hline & 254 & Linke $\mathrm{H}$. & 278 \\
\hline 238 , & 239 & Linke W.A. & $256,257,266$ \\
\hline & 274 & Llinas P. & 242 \\
\hline 263 , & 264 & Lochnit G. & 276 \\
\hline & 274 & Lombardi V. & 258,257 \\
\hline & 284 & Lombardo A.T. & 237 \\
\hline & 248 & Longa E. & 244,273 \\
\hline & 245 & López de Munain A. & 282 \\
\hline & 251 & Lorenzi M. & 261 \\
\hline & 245 & Lösel D. & 236 \\
\hline & 265 & Lossie J. & 241 \\
\hline 238 , & 239 & Lo Verso F. & 273,244 \\
\hline & 282 & Lubomirov L.T. & 259 \\
\hline & 241 & Lucas B. & 248 \\
\hline & 277 & Luiking Y. & 272 \\
\hline 251 , & 253 & Lukacs P. & 282 \\
\hline & 277 & Łukasz M. & 263 \\
\hline & 278 & Lundy S. & 277 \\
\hline 250, & 255 & Luther P.K. & 267,268 \\
\hline & 250 & Lyon A.R. & 256 \\
\hline & 256 & & \\
\hline & 242 & MacIntosh B.R. & 262 \\
\hline & 275 & Mackey A.L. & 237 \\
\hline & 263 & MacLeod K. & 256 \\
\hline 238 , & 261 & Maden-Wilkinson T.M. & 246,249 \\
\hline & 275 & Maffei M. & 244 \\
\hline & 266 & Maganaris C.N. & 283 \\
\hline & 253 & Maier A.B. & 255 \\
\hline & 273 & Malik F.I. & 269 \\
\hline & 261 & Málnási-Csizmadia A. & 263,264 \\
\hline & 278 & Manders E. & 269 \\
\hline & 282 & Manfredi L.H. & 274 \\
\hline & 281 & Månsson A. & 278 \\
\hline & 267 & Mansfield C. & 267 \\
\hline & 257 & Maridaki M. & 275 \\
\hline & 257 & Marston S. & 267 \\
\hline & & Marston S.B. & 268 \\
\hline & 265 & Martinez Molina D. & 240 \\
\hline & 272 & Martinez-Morentin L. & 264 \\
\hline & 244 & Martucci E. & 255 \\
\hline & 275 & Masiero E. & 273 \\
\hline 259,260 , & 262 & Mattiello S.M. & 283 \\
\hline & 256 & Mattiello-Sverzut A.C. & $263,264,283$ \\
\hline & 268 & Maughan D.W. & 237 \\
\hline & 278 & Mauseth M.A. & 264 \\
\hline 260, & 262 & McDonald F.B. & 279 \\
\hline & 265 & McPhee J. & 268 \\
\hline & 261 & McPhee J.S. & 246,249 \\
\hline & 280 & Melli L. & 257 \\
\hline
\end{tabular}


Mendler L.

Meregalli M.

Metalnikova N.A.

Michels M.

Mike A.K.

Minozzo F.

Minozzo F.C.

Miotti D.

Miranda J.I.

Mitchell W.W.A.

Mokbel N.

Molina B.

Mollica J.P.

Morano I.

Moro T.

Moroz N.

Morris E.

Morris E.P.

Moss R.L.

Mouly V.

Mugelli A.

Müller A.E.

Müllerova D.

Mullerova D.

Murphy A.M.

Murphy R.M.

Musarò A.

Mutungi G.

Myatt J.

Myburg K.H.

Naber N.

Nagel J.

Nagel-Steger L.

Nakai A.

Narayanan $\mathrm{O}$.

Narici $M$.

Narici M.V.

Natanek S.A.

Navegantes L.C.C.

Negroni E.

Neilson J.

Neuhold J.

Neumann J.

Neunhäuserer D.

Nevzorov I.A.

Nezos A.

Nguyen-McCarty M.

Nica A.S.

Nishizawa S.

Noble K.

Nordlund P.

Novak S.M.

Nowakowski S.G.

Nworu C.U.

Ocoor K.

Odom G.L.

Offringa $\mathrm{C}$.

Ogut $\mathrm{O}$.

O'Halloran K.D.

Ohira T.

Ohira Y.

Ohlsson M.

Ohno Y.
238, 239

279

241

244

282

267

254

239

282

250

251

264

244

241

239, 240

255

251

268

266

272, 278

279

266

240

261

243

244

272, 281

282

252

277

243

272

256

275

242

273

246, 249, 268

274

274

278

280

261

274

250

247

275

284

241, 249

275

281

240

249

236, 277

250, 253

\section{4}

277

273

243

279

275

275

277

275
Oldfors A.

Olivieri F.

Olivotto I.

Onipe A.

Ono Y.

Ørtenblad N.

Oshiro N.

Ottenheijm C.

Ottenheijm C.A.

Ottenheijm C.A.C.

Paalberends E.R.

Pace N.

Pacelli F.Q.

Palm L.

Palmer B.M.

Paltineanu B.

Paoli A.

Paolini C.

Papp Z.

Pappas C.T.

Parolini D.

Patchell V.B.

Pate E.

Pecchi E.

Peijian Z.

Pellegrino M.A.

Peltonen J.

Pena C.

Peña Paz M.I.

Perera S.

Persson M.

Petzhold D.

Pfitzer G.

Pfuhl M.

Philippou A.

Piazzesi G.

Piccirillo R.

Piechura $\mathrm{H}$.

Pinotsis N.

Pirker K.

Piroddi N.

Poggesi C.

Polkey M.I.

Pöllänen E.

Prada G.

Prada S.

Prado C.M.

Preissner K.T

Prescott J.

Prochniewicz E.

Procopio A.D.

Protasi F.

Puetz S.

$248,251,253,255,261,270$

253

242,246

242, 246, 279

274

238,262

241, 249

241, 249

264

276

280

266

261

259

259

Radzyukevich T.

274

Rain S.

Rassier D.E.

Rauscher A.Á.

Razini P.

Reconditi $\mathrm{M}$.

Redfield M.M.

Rędowicz M.J.

Redwood C.S.

Reggiani C.

277

279

248

245

274

269

244

246

277

237

259

242

279

50

83

257

273

249
240

265
278

241

259

275

269

$267,252,254$

264

279

258

257

263

247

239, 240, 259, 260, 262
269

258

276

9

Sch

\section{$\mathrm{S}$}

Schnurr D.C.

Schrøder H.D.

Schreiner C.

Schroeter M.M.

Scorzeto M.

Segura-Alegria B.

Serrão P.R.

Sethi R.

Seynnes O.

Seynnes O.R.

Shah S.

Sikkel M.

Sipilä S.

Sitzia C.

Sjoeblom B.

Sjøgaard G.

Smale S.T.

Song L.

Song W.

Soraru G.

Sorimachi H.

Soukup T.

Sparrow J.

Squire J.M.

Stenroth L.

236, 277

241, 249

241, 249

263, 264 
Stienen G.

Stienen G.J.M.

Suetta C.

Suffredini S.

Sumida J.

Sun Y.B.

Suuronen E.J.

Suzuki M.

Svergun D.

Svergun D.I.

Swank D.

Sweeney H.L.

Szentesi P.

Tajsharghi $\mathrm{H}$.

Takao S.

Takaya E.

Tanner B. C.W.

Tardiff J.C.

Taube M.

te Kronnie G.

ten Siethoff L.

tenCate F.J.

Tesi C.

Testerink J.

Theos A.

Thiele F.

Thomas D.D.

Tian R.

Todt $\mathrm{H}$.

Toepfer C.

Toniolo L.

Toral I.

Torrente Y.

Tosi B.

Tremble S.M.

Trinick J.

\begin{tabular}{|c|c|c|}
\hline & 265 & Trisolino G. \\
\hline $243,244,259$, & 269 & Tsaturyan A.K. \\
\hline & 245 & Tsiavaliaris G. \\
\hline & 279 & Tsukada T. \\
\hline & 246 & Turner N. \\
\hline & 265 & Turton P. \\
\hline & 281 & \\
\hline & 275 & Udd B. \\
\hline & 251 & Ushida $\mathrm{T}$. \\
\hline & 253 & \\
\hline & 239 & Vallejo-Illarramendi A. \\
\hline & 242 & Vallese D. \\
\hline & 242 & $\begin{array}{l}\text { van der Velden J. } \\
\text { van de Vyver M. }\end{array}$ \\
\hline & 277 & van der Laarse W.J. \\
\hline & 275 & Van der Ven P. \\
\hline & 251 & van der Ven P.F. \\
\hline & 237 & Van Dijk M. \\
\hline & 250 & Van Norren K. \\
\hline & 241 & van Slegtenhorst M. \\
\hline & 278 & VanBuren P. \\
\hline & 278 & Várkuti B.H. \\
\hline & 244 & Vasilceac F.A. \\
\hline 242,246 & 279 & Vasuri E. \\
\hline & 273 & Vazirpanah N. \\
\hline & 275 & Vergani L. \\
\hline & 239 & Verma N.K. \\
\hline 264, & 266 & Vidal-Pelissier M.C. \\
\hline & 277 & Vihola A. \\
\hline & 282 & Vilmen C. \\
\hline $252,256,267$, & 268 & Vitiello L. \\
\hline 239,240 & 259 & Voelkel T. \\
\hline & 282 & Voit T. \\
\hline & 279 & Vonk A.-Noordegraaf \\
\hline & 279 & Vonk-Noordegraaf A. \\
\hline & 237 & Vydyanath A. \\
\hline
\end{tabular}

248,252
255, 268

241, 242

283

250, 255

251

280

284

275

282

278

$243,, 244$

277

273

245

253

272

272

244

237

263, 264

283

255

244

275

259

257

284

280

278

256

271

269

269

267
Wada S.

Wadley G.

Wang Y.

Wardle F.

Warscheid B.

Weeks A.

Weiss R.

West T.

Westerblad H.

Westerhof N.

Wijnker P.J.M.

Wilmanns M.

Wilson A.

Winstanley C.

Woledge R.

Wójtowicz I.

Wray S.

Yamamoto D.

Yang $\mathrm{Z}$.

Yao L.

Yasuhara $\mathrm{K}$.

Ylänne J.

Yoshioka T.

Yuen S.

Ywazaki J.L.

Zádor E.

Zaleska M.

Zanon N.

Zarnescu D.C.

Zoldak G.

Zong A.

Žurmanová J.
275

275

237, 256

247

259

281

277

252, 267

260

269

243

248, 251, 252, 257

252

278

252

261

$278,280,281$

259

264

279

275

237

275

243

263

278

247

274

249

251

242

260 